\title{
WIND ENERGY FORECASTS IN CALCULATION OF EXPECTED ENERGY NOT SERVED
}

By

Richard Sun

Bachelor of Applied Science and Engineering, University of Toronto 2011

\author{
A thesis \\ presented to Ryerson University \\ in partial fulfillment of the \\ requirements of the degree \\ Master of Applied Science \\ in the program \\ Electrical and Computer Engineering \\ Toronto, Ontario, Canada, 2014
}

CRichard Sun 2014 


\begin{abstract}
AUTHOR'S DECLARATION FOR ELECTRONIC SUBMISSION OF A THESIS I hereby declare that I am the sole author of this thesis. This is a true copy of the
\end{abstract} thesis, including any required final revisions, as accepted by my examiners.

I authorize Ryerson University to lend this thesis to other institutions or individuals for the purpose of scholarly research

I further authorize Ryerson University to reproduce this thesis by photocopying or by other means, in total or in part, at the request of other institutions or individuals for the purpose of scholarly research.

I understand that my thesis may be made electronically available to the public. 
Wind Energy Forecasts In Calculation of Expected Energy Not Served

Master of Applied Science 2014

Richard Sun

Electrical and Computer Engineering

Ryerson University

\section{ABSTRACT}

The stochastic nature of wind energy generation introduces uncertainties and risk in generation schedules computed using optimal power flow (OPF). This risk is quantified as expected energy not served (EENS) and computed via an error distribution found for each hourly forecast. This thesis produces an accurate method of estimating EENS that is also suitable for real-time OPF calculation.

This thesis examines two statistical predictive models used to forecast hourly production of wind energy generators (WEGs), Markov chain model, and auto-regressive moving-average (ARMA) model, and their effects on EENS. Persistence model is used as a benchmark for comparison. For persistence and ARMA models, both Gaussian and Cauchy error distributions are used to compute EENS via a closed-form solution that reduces computational complexity..

Markov chain and ARMA both provide accurate forecasts of WEG power generation though Markov Chain model performs significantly better. The Markov chain model also produces the most accurate EENS estimate of the three models. 


\section{ACKNOWLEDGEMENTS}

I would like to express my sincere gratitude and appreciation for my supervisors, Dr. Bala Venkatesh and Dr. Soosan Beheshti. Their support, patience, and guidance have been instrumental to this research and their insight and knowledge have been invaluable to my own personal development.

I would also like to express my gratitude for the endless encouragement given by my parents who are a constant inspiration and an object lesson that hard work is the key to achieving anything worth having.

Finally, I would like to thank my instructors and colleagues at Ryerson University and especially members of the Centre for Urban Energy for providing a wonderful working environment throughout this research. 


\section{TABLE OF CONTENTS}

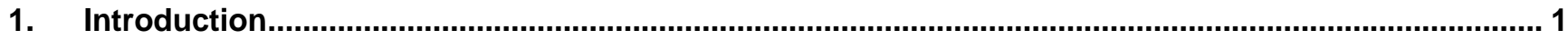

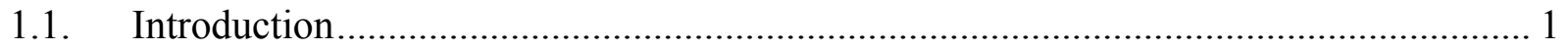

1.2. Survey of Recent Work in WEG Forecasting and EENS ......................................... 1

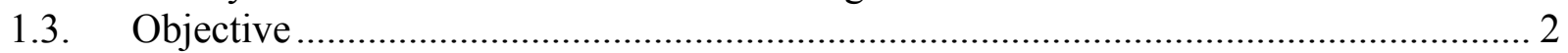

1.4. Chapter-wise Introduction ........................................................................... 3

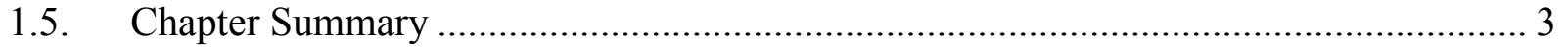

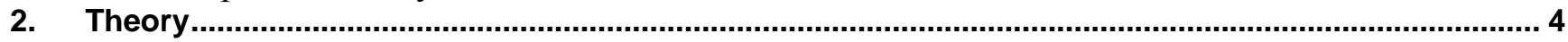

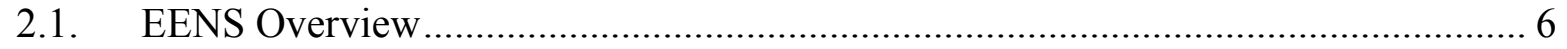

2.2. Persistence Model (Used as a benchmark) ............................................................... 8

2.2.1. Theoretical Background ............................................................................. 8

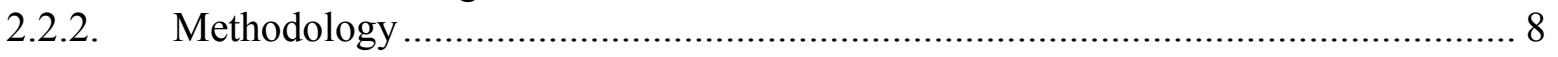

2.2.3. Probability Density Function .................................................................. 9

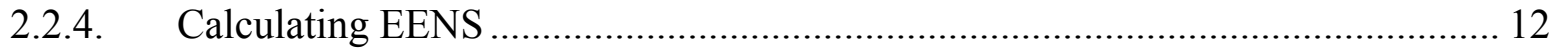

2.3. Markov Chain Model ......................................................................................... 15

2.3.1. Theoretical Background .............................................................................. 15

2.3.2. Methodology ....................................................................................... 19

2.3.3. Probability Density Function ................................................................... 19

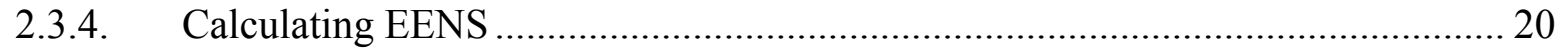

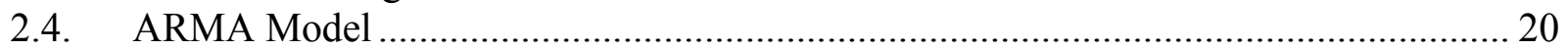

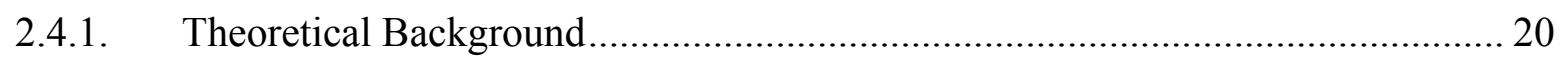

2.4.2. Model Order............................................................................................... 22

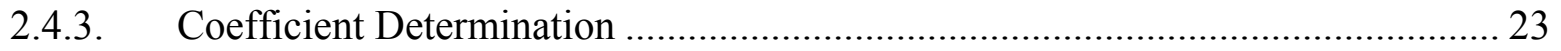

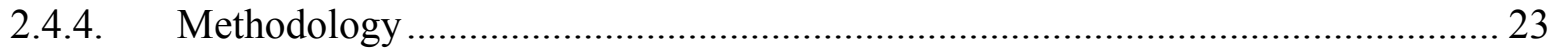

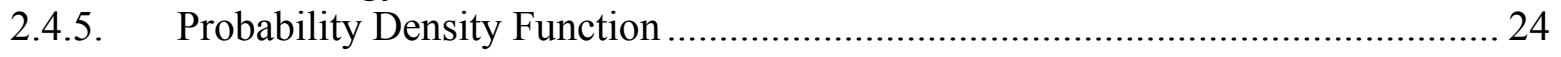

2.4.6. Calculating EENS ............................................................................... 24

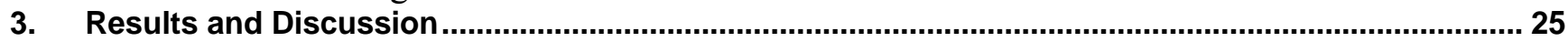

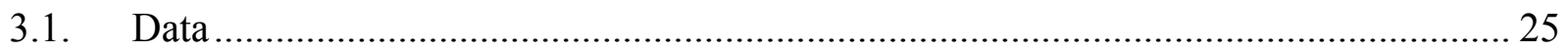

3.2. Amaranth Wind Farm ................................................................................ 25

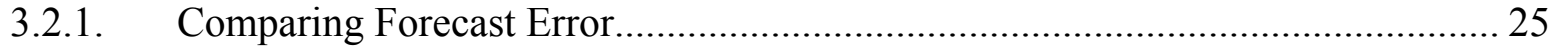

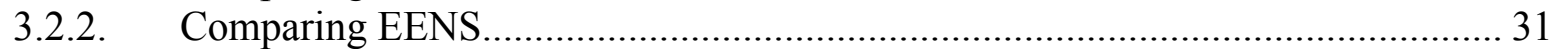

3.3. Wolfe Island Wind Farm ........................................................................... 38

3.3.1. Comparing Forecast Error......................................................................... 38

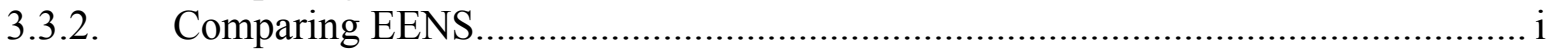

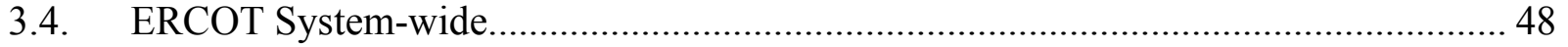

3.4.1. Comparing Forecast Error.......................................................................... 48

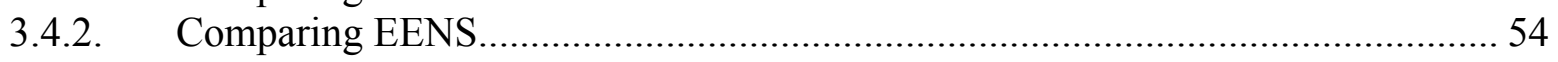

3.4.3. Comparison with Previous Work ................................................................ 55

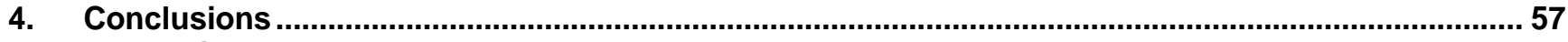

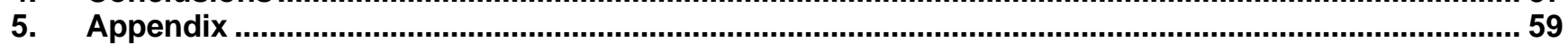

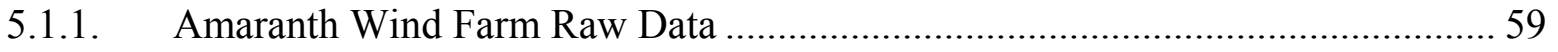

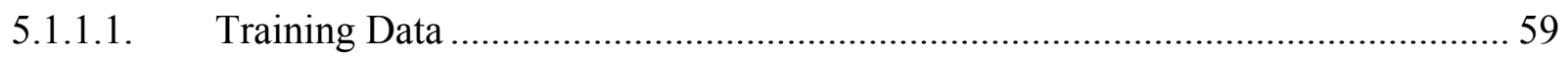

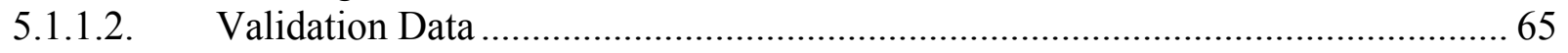

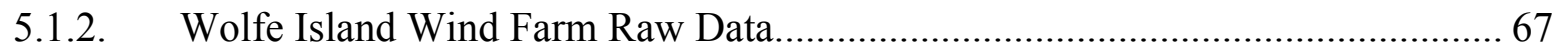




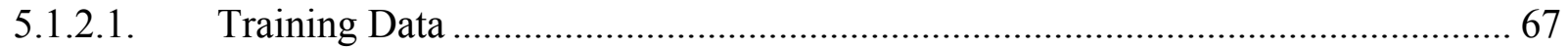

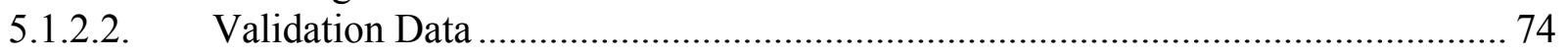

5.1.3. ERCOT System-wide Raw Data.................................................................... 75

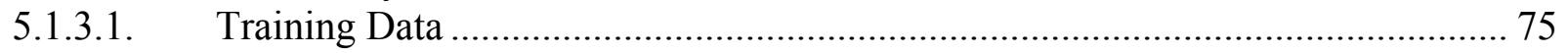

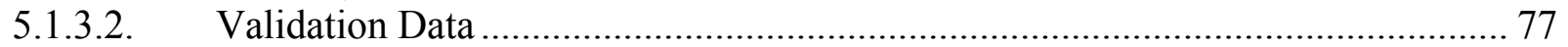

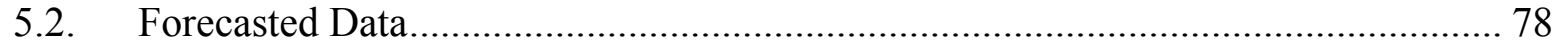

5.2.1. Amaranth Wind Farm Forecasted Data .......................................................... 78

5.2.2. Wolfe Island Wind Farm Forecasted Data..................................................... 88

5.2.3. ERCOT System-wide Forecasted Data......................................................... 97

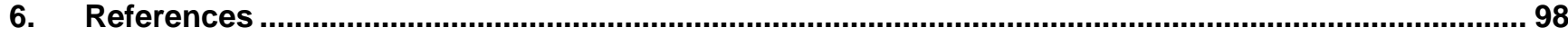




\section{LIST OF TABLES}

Table 3.2.1.1: NRMSE of forecast for each predictive model (Amaranth)

Table 3.2.2.1: NRMSE of EENS vs. AENS for each predictive model and distribution

(Amaranth).

Table 3.3.2.1: NRMSE of forecast for each predictive model (Wolfe Island)

Table 3.3.1.1: NRMSE of EENS vs. AENS for each predictive model and distribution (Amaranth)

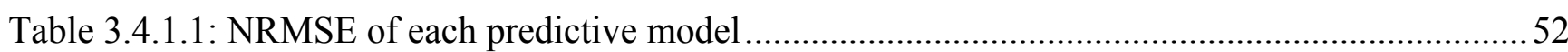

Table 3.4.3.1: Accuracy with 25\% error limit for one hour ahead forecast in [11] ................................55

Table 3.4.3.2: Accuracy with 25\% error limit for one hour ahead forecast in for persistence, Markov chain, and ARMA models. 


\section{LIST OF FIGURES}

Figure 2.2.3.1: Diagram of the EENS calculator ...........................................

Figure 2.2.3.1: Forecasting Error Probability Density Function ................................11

Figure 2.3.1.1: Example of a transition matrix created by a Markov chain model with $N=20 \ldots 16$

Figure 2.3.1.2: Sample two state Markov chain model.......................................18

Figure 2.4.1.1: Flowchart depicting the process of determining.............................21

Figure 3.2.1.1: Persistence model wind energy generation forecast compared to actual

generation for Amaranth Wind Farm ......................................................26

Figure 3.2.1.2: Markov chain model wind energy generation forecast compared to actual

generation for Amaranth wind farm ..................................................27

Figure 3.2.1.3: ARMA model wind energy generation forecast compared to actual generation for

Amaranth wind farm............................................................................28

Figure 3.2.1.4: Forecast errors of all three predictive models plotted together for Amaranth wind

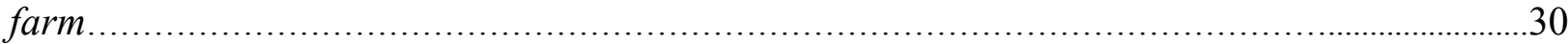

Figure 3.2.2.1: EENS calculated from persistence, Markov Chain, and ARMA models for

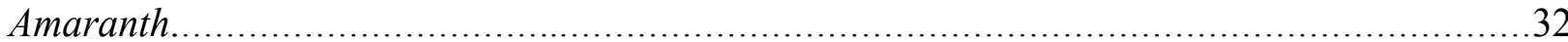

Figure 3.2.2.2: EENS plotted with AENS for persistence model with PG at 96MW for Amaranth

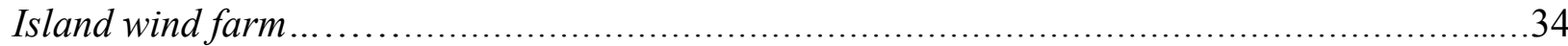

Figure 3.2.2.3: EENS plotted with AENS for Markov chain model with PG at 96MW for

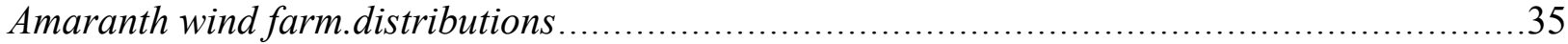

Figure 3.2.2.4: EENS plotted with AENS for ARMA model with PG at 96MW for Amaranth

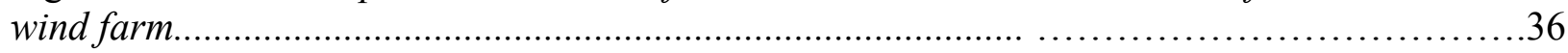

Figure 3.3.1.1: Persistence model wind energy generation forecast compared to actual generation for Wolfe Island wind farm. ...............................................................39

Figure 3.3.1.2: Persistence model wind energy generation forecast compared to actual generation for Wolfe Island wind farm .....................................................40

Figure 3.3.1.3: Persistence model wind energy generation forecast compared to actual generation for Wolfe Island wind farm.

Figure 3.3.1.4: Forecast errors of all three predictive models plotted together for Wolfe Island wind farm. ................................................................................................43

Figure 3.3.2.1: EENS calculated from persistence, Markov Chain, and ARMA models for Wolfe

Island wind farm. .................................................................................44

Figure 3.3.2.2: EENS plotted with AENS for persistence model with PG at 90MW for Wolfe

Island wind farm. . ............................................................................. 45

Figure 3.3.2.3: EENS plotted with AENS for Markov chain model with PG at 90MW for Wolfe

Island wind farm. ................................................................................46

Figure 3.3.2.4: EENS plotted with AENS for ARMA model with PG at 90MW for Wolfe Island

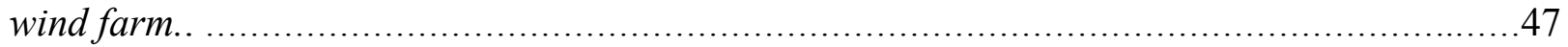

Figure 3.4.1.1: Persistence model wind energy generation forecast compared to actual generation for ERCOT system-wide.

Figure 3.4.1.2: Markov chain model wind energy generation forecast compared to actual

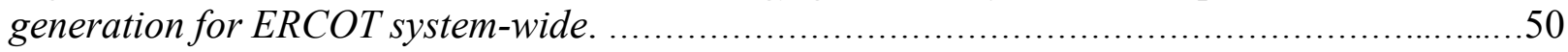
Figure 3.4.1.3: ARMA model wind energy generation forecast compared to actual generation for ERCOT system-wide. 
Figure 3.4.1.4: Forecast errors of all three predictive models plotted together for ERCOT system-wide.

Figure 3.4.2.1: EENS calculated from persistence, Markov Chain, and ARMA models for ERCOT system-wide. 


\section{LIST OF APPENDICES}

5.1.1. Amaranth Wind Farm Raw Data .................................................................. 59

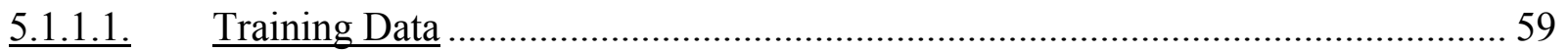

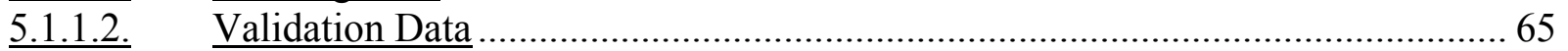

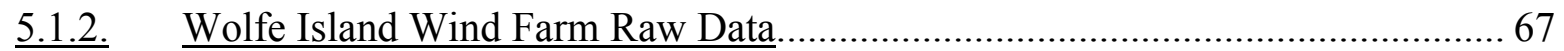

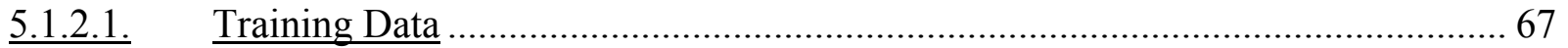

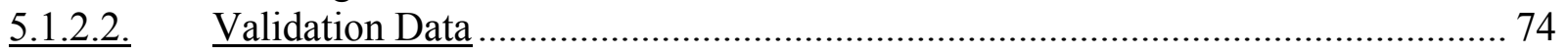

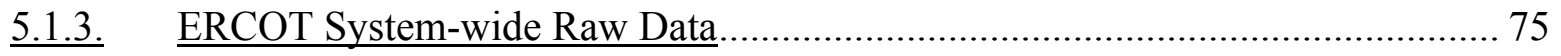

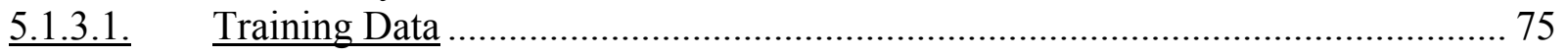

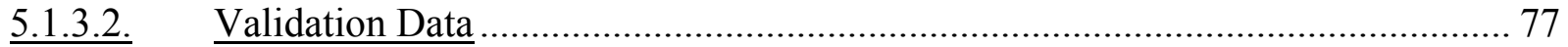

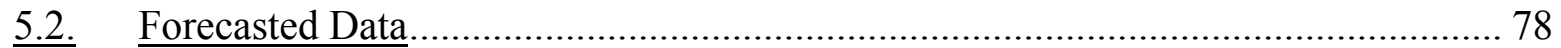

5.2.1. Amaranth Wind Farm Forecasted Data ........................................................ 78

5.2.2. Wolfe Island Wind Farm Forecasted Data........................................................ 88

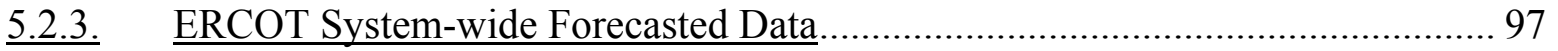




\section{General Terminology}

\section{List of Symbols}

$\hat{X}_{t}:$ a time series of the forecasted wind energy generation.

$X_{t}$ : a time series of the actual wind energy generation.

$P G_{t}$ : A random variable that describes WEG generation at time $t$.

$n$ : the number of elements within the training data.

$m$ : the number of elements within the validation data.

$P G_{\text {nominal: }}$ nominal wind power. For this case it is the maximum wind power a generator is capable of outputting.

$\overline{\overline{P G_{t}}}$ : the forecasted power generation of a WEG at time $t$.

$\beta$ : the PDF scale parameter in the general case.

$P G_{\text {sched: }}$ the power generation scheduled for a given point of time.

$\Delta t$ : the time interval used between forecasts. For this case it is one hour.

Markov Chain Model Terminology

$N$ : the number of states used in the Markov chain model

$S_{i}:$ the $i^{\text {th }}$ state of a Markov chain

$P_{i}$ : the amount of power generation that corresponds to the $i^{\text {th }}$ state of a Markov chain

$\mathbf{P}\left(t_{h}\right)$ : the first-order Markov chain transition matrix

$\hat{\boldsymbol{P}}\left(t_{h}\right)$ : the estimate of the first-order Markov chain transition matrix

$p_{i j}\left(t_{h}\right)$ : the generic element of $\mathbf{P}\left(t_{h}\right)$

$p_{i}\left(t_{h}\right)$ : the generic column of $\mathbf{P}\left(t_{h}\right)$

$p_{j}\left(t_{h}\right)$ : the generic row of $\mathbf{P}\left(t_{h}\right)$

ARMA Model Terminology

$A R M A(p, q)$ : an ARMA process with orders $p$ and $q$ 
$p$ : the order of the auto-regressive part of an ARMA process

$q$ : the order of the moving-average part of an ARMA process

$a_{j}$ : the coefficients of the auto-regressive part of an ARMA process

$b_{k}$ : the coefficients of the moving-average part of an ARMA process 


\section{List of Abbreviations}

WEG: Wind Energy Generator

OPF: Optimal Power Flow

AENS: Actual Energy Not Served

EENS: Expected Energy Not Served

PDF: Probability Distribution Function

ARMA: Autoregressive-moving average

ANN: Artificial Neural Network

NRMSE: Normalized Root-Mean-Square 


\section{INTRODUCTION}

\subsection{Introduction}

When wind energy generators (WEGs) are incorporated into the power supply mix, the uncertainties associated with wind energy increases the risk of power shortages and failure to supply a contracted load. This risk is also carried into determining optimal power flow (OPF) and is quantified by expected energy not served (EENS). EENS is an estimate of actual energy not served (AENS) which is the actual energy shortfall of a WEG at a given point in time. At any given time, a certain amount of power generation is scheduled from every WEG which is denoted here as $P G_{\text {sched. }}$ EENS is the expected energy shortfall from the scheduled power. This shortfall is probabilistic because of the stochastic nature of wind energy and the uncertainties of the wind energy forecast.

Having an accurate forecast of WEG output and being able to calculate EENS in a timely fashion is very important for estimating the costs associated with uncertainties from WEGs as they become a larger part of the power grid.

\subsection{Survey of Recent Work in WEG Forecasting and EENS}

In the past, Monte Carlo simulation has been used to stochastically model WEG integration to the grid and to estimate the costs associated with the uncertainty introduced by the WEGs as in [1]. Ref. [2] uses Monte Carlo simulation in conjunction with OPF to maximize

social welfare as it pertains to uncertainties in WEG generation. Ref. [3] uses Monte Carlo simulation to stochastically model locational marginal prices (LMP) and examine the effect that 
the introduction of WEG has on LMP. Monte Carlo simulation however, can be very taxing computationally, especially in systems with large numbers of WEGs as it scales exponentially with the number of buses in the system. Alternative ways of quantifying EENS have been developed for OPF as a result. Ref. [4] introduces a triangular-approximation for modeling WEG for OPF. Approximating the probability distribution of WEG generation effectively linearizes it, making it readily usable with OPF and does not require time-consuming Monte Carlo simulation. Thus, the method introduced in [4] is suitable for real-time OPF applications where the methods using Monte Carlo simulation are not. One shortcoming of this method however is that it uses an estimate of the WEG forecast's PDF and thus the accuracy of EENS estimation will suffer.

\subsection{Objective}

The main motivation of this thesis is to develop a methodology to estimate EENS from a predictive model that is suitable for real-time OPF. The relative accuracy of two statistical-only predictive models, autoregressive-moving-average (ARMA), and Markov chain as well as their effect on EENS calculations will also be examined. The effects of using Gaussian error distributions and Cauchy distributions to calculate EENS will also be analyzed.

This thesis will develop a way of estimating EENS using closed-form or pseudo-closedform equations for EENS calculations. This will produce a more accurate estimate of EENS than in [4] because it dispenses with the approximation and will also be suited for real-time OPF applications. 


\subsection{Chapter-wise Introduction}

\section{Chapter 2:}

Chapter 2 discusses the background of WEG forecasting and introduces the two models that are to be studied, Markov chain and ARMA as well as persistence model which is to be used as a benchmark. Background and calculation of EENS is discussed in the general case. For each model, the forecasting mechanism is explored in detail as well as the method by which a PDF is derived for EENS calculation. Finally, calculation of EENS is discussed for each model.

\section{Chapter 3:}

Chapter 3 presents the simulation results for each model. The accuracy of the forecast produced by each model is evaluated. The EENS estimated by each model is also examined and evaluated. Three case studies are considered: Amaranth wind farm, Wolfe Island wind farm, and ERCOT system-wide.

\section{Chapter 4:}

Chapter 4 presents the conclusions that can be drawn from this work. Recommendations for which model and PDF should be used for EENS estimation are given based on the results presented in chapter 3. The main contributions and relevance of this work are also discussed.

\subsection{Chapter Summary}

This chapter presents an introduction to the topic, motivation and objective of this thesis. It is followed by a chapter-wise summary. 


\section{THEORY}

EENS is calculated from a probability distribution of the error in the forecasted WEG output. Thus, in order to determine EENS, two things are required: a forecast for wind energy output, and an error distribution of the forecast. The forecast for wind energy output at time $t$ is denoted by $\hat{X}_{t} . X_{t}$ is the actual wind energy generation of the next time step at time $t$ and is used to determine the accuracy of the forecast. This thesis will explore three different predictive models for forecasting WEG output as well as methods of deriving a probability distribution of the error so that EENS can be calculated.

There are two main categories of predictive models for wind power generation: physical and statistical. Physical models consider physical variables such as wind speed, temperature, atmospheric conditions, etc. Statistical models do not consider any physical variables and instead use time-series analysis of historical wind power generation data. Statistical models have the advantage of being computationally simpler as well as being easier to implement than physical models.

Two predictive models will be explored, Markov chain, and ARMA. Markov chain and ARMA model were chosen for their accuracy on shorter time horizons as well as the advantages afforded by the fact that they are purely statistical models[5][6]. The Markov chain model in particular produces a very accurate probability density function for the forecasting error which is very useful in calculating EENS[6]. These two models will be used to generate forecasts for WEG outputs on the one-hour-ahead timescale. A third model, persistence model, will also be used as a benchmark for the first two. ARMA and Markov chain models have been compared 
and evaluated in the past for their forecasting ability but their respective effects on EENS have $\operatorname{not}[7]$.

An artificial neural network (ANN) is another statistical-only model that as become an increasingly popular method of forecasting WEG generation. ANNs are models that are sonamed because they attempt to mimic the structure and functionality of animal brains. Adaptive weights are used to conceptualize the relative strengths of the connections within the network. They are trained by a training data set and used in prediction.

ANNs can be generally classified as feed-forward or feedback [8]. Feed-forward ANNs are more common in WEG forecasting and include backpropagation and radial bases function [9]. Backpropagation refers to the error of the ANN which is backpropagated through the network so that the weights can be updated in order to minimize future errors [10].

ANNs are capable of producing a point-forecast and associated probability distribution that is suitable for EENS estimation but will not be discussed in detail within this work. However, the method of estimating EENS from a forecasting model established in this work can be used with ANNs as well as other statistical models.

The next step in calculating EENS is to generate a probability distribution for these forecasts. In the case of the Markov chain model, a probability distribution will be created by the model itself for each forecast. In the case of persistence and ARMA, the probability distribution is modeled by a Gaussian or a Cauchy distribution. The scale parameters for each distribution will be determined by historical data.

There are several statistical distributions that can be used to model the error distribution of wind power generation point forecasts. As discussed previously, the forecast must be able to 
generate a probability distribution for a given time in order for EENS to be calculated. [11] provides a method for calculating EENS using a Weibull distribution for wind speed. Gaussian and Cauchy distributions will be utilized in this thesis and they will be fitted to WEG output generation as opposed to wind speed. For shorter time-steps, on the order of 1 hour, a Cauchy distribution will often provide a better fit than Gaussian distribution. This is a result of the excess kurtosis exhibited by the observed error distribution of wind energy forecasts. The observed error distributions have higher peaks and shorter tails than the fitted Gaussian has and are closer in appearance to Cauchy distributions. In many instances however, the central-limit theorem can be invoked to justify the use of a Gaussian distribution.

\subsection{EENS Overview}

The EENS of the WEG is the amount of energy that is expected to be less than the scheduled generation $P G_{\text {sched }}$ based on the probability distribution of the forecast error. In the general case, EENS can be calculated from:

$$
\text { EENS }=\int_{0}^{P G_{\text {sched }}}\left(P G_{\text {sched }}-P G\right) \cdot P D F(\overline{\overline{P G}}, \beta) d P G
$$

$\operatorname{PDF}(\overline{\overline{P G}}, \beta)$ in equation (2.3.1) above differs depending on the PDF being used. The Gaussian and Cauchy distributions each have a location parameter and a scale parameter which are denoted in the general case by $\overline{\overline{P G}}$ and $\beta$, respectively. In both Gaussian and Cauchy distributions the location parameter is $\overline{\overline{P G}}$. The scale parameter for the Gaussian distribution is $\sigma^{2}$ and the scale parameter for the Cauchy distribution is $\gamma$. The location parameter $\overline{\overline{P G}}$ is the point 
forecast produced by the forecasting model. The scale parameters are produced by the forecasting model using the training data. These two parameters are required for EENS to be calculated. The specifics of determining the location and scale parameters and calculating EENS from it in the case of Gaussian and Cauchy distributions will be described in detail in section 2.2.3, and 2.2.4, respectively.

In the case of the Markov chain model, the model produces a discrete PDF which is immediately suitable for EENS calculation. The specifics of producing the PDF in the this case and calculating EENS from it will be described in detail in sections 2.3.3, and 2.3.4, respectively.

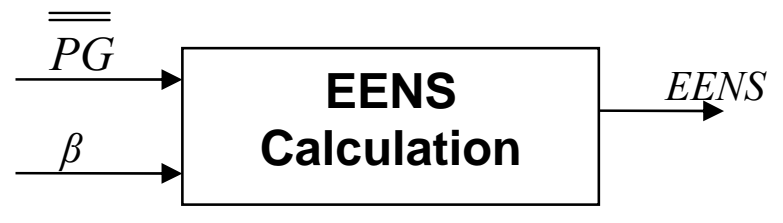

Figure 2.1.1: Diagram of the EENS calculator

Figure 2.1.1 shows a visual representation of the method outlined in this section. The "EENS Calculation" box represents equation 2.3.1. It can be seen from figure 2.1.1 that EENS is a function of $\overline{\overline{P G}}$ and $\underline{\beta}$ both of which are produced by the forecasting model. 


\subsection{Persistence Model (Used as a benchmark)}

\subsubsection{Theoretical Background}

The persistence model assumes that the wind generation at the next hour is equal to the generation at the current hour. The forecasted generation at the next time step can be given by [12]:

$$
\hat{X}_{t}=X_{t-1}
$$

For very short time-steps (on the order of minutes) persistence provides a very accurate forecast and is in fact difficult to improve upon. For short time-steps (on the order of an hour) it still provides an adequate forecast. This is because there is high correlation between wind speeds[3], that is, wind speed does not change much from hour to hour and even less so from minute to minute.

Because persistence is very simple and easy to implement, it is often used as a benchmark to compare with other forecasting models. Other forecasting models must be able to outperform persistence to be viable.

\subsubsection{Methodology}

As seen in equation (2.2.1.1), the forecasted generation $\hat{X}_{t}$ at time $t$ is given by the actual generation of the previous time step, $X_{t-1}$. The probability density function (PDF) of the forecasting error gives the probability distribution of forecasted power generation $\hat{X}_{t}$ at the next time step. 


\subsubsection{Probability Density Function}

As noted previously, a probability distribution of a given point forecast's error (error PDF) is required for EENS calculation. In the case of the persistence and ARMA model, the error PDF is assumed to be either a Gaussian distribution or a Cauchy distribution.

Two parameters must be determined to construct the error PDF: a location parameter, and a scale parameter. The location parameter is the forecast at the current time step in both cases. The scale parameter is $\sigma^{2}$ for the Gaussian distribution and $\gamma$ is the scale parameter for the Cauchy distribution. EENS is a calculated as a function of this location parameter, and scale parameter.

Gaussian: For a Gaussian distribution, the PDF is given by:

$$
P D F_{G}\left(P G_{t}\right)=\frac{1}{\sqrt{2 \pi \sigma^{2}}} \exp \left(\frac{-\left(P G_{t}-\overline{\overline{P G_{t}}}\right)}{2 \sigma^{2}}\right)
$$

$P G_{t}$ ranges from 0 to $P G_{\text {nominal. }} \overline{\overline{P G_{t}}}$ is the forecasted generation of the current time step. It is the mean of the Gaussian distribution and the location parameter of the Cauchy distribution. Thus, it is given by:

$$
\overline{\overline{P G_{t}}}=\hat{X}_{t}
$$

$\hat{X}_{t}$ can be found from equation (2.2.1.1). 
$\sigma^{2}$ is the scale parameter of the Gaussian distribution and also the variance of the difference between the forecasted generation and the actual generation at each time step during the training data period. $\sigma^{2}$ is given by:

$$
\sigma^{2} \frac{1}{n} \sum_{t=1}^{n}\left(Y_{t}-\hat{\hat{Y}}_{t}\right)^{2}
$$

Where $n$ is the number of elements in the training data, $Y_{t}$ is a time series of training data and $\hat{Y}_{t}$ is a time series of the prediction of the training data.

Cauchy: In the case of a Cauchy distribution, the PDF is given by:

$$
P D F_{C}\left(P G_{t}\right)=\frac{1}{\pi \gamma\left[1+\left(\frac{P G_{t}-\overline{\overline{P G_{t}}}}{\gamma}\right)^{2}\right]}
$$

$\gamma$ is selected to be half of the interquartile range of the Gaussian distribution and is thus given by:

$\gamma=0.6745 \sigma$

With the location parameter $\overline{\overline{P G_{t}}}$, and the scale parameters $\sigma^{2}$ and $\gamma$ for Gaussian and Cauchy respectively, EENS can be calculated. 


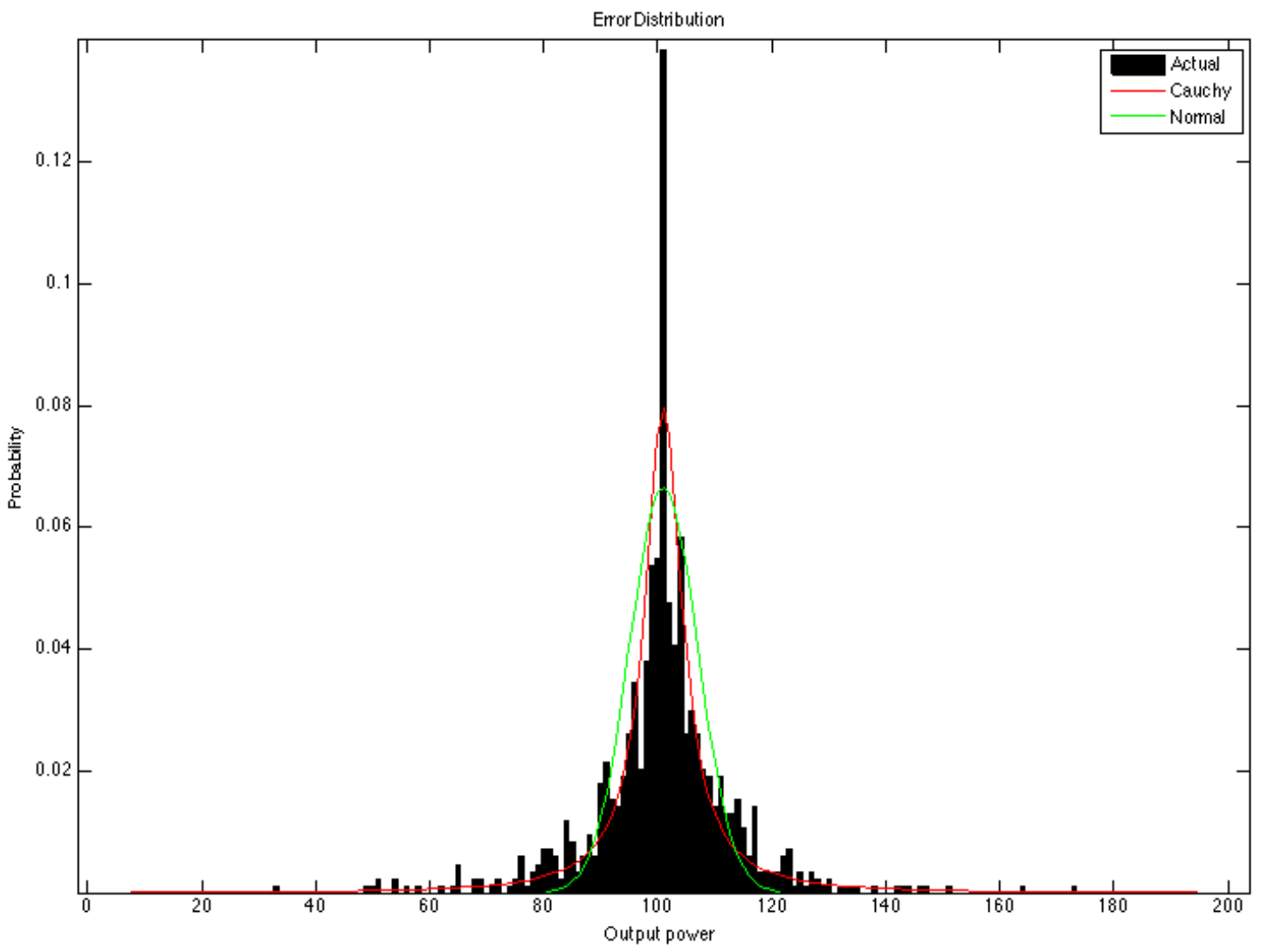

Figure 2.2.3.1: Forecasting Error Probability Density Function

Figure 1 shows the actual error distribution determined from a persistence model over the training data period. A Gaussian and Cauchy distribution based on a forecast of $\overline{\overline{P G_{t}}}$ at $100 \mathrm{MW}$ is overlayed on it. A bin size is chosen such that the number of bins is larger than the minimum prescribed by Scott's normal reference rule. The data comes from 3960 hours of wind generation data from the Amaranth wind farm. 


\subsubsection{Calculating EENS}

As discussed previously, in the case of persistence and ARMA models, Gaussian and

Cauchy distributions are used to fit the probability density functions of the forecast and to calculate EENS.

Gaussian: A solution for EENS calculated with a Gaussian distribution at a given $P G_{s c h e d}$ is derived below:

$$
\begin{aligned}
& \text { EENS }_{G}=\frac{1}{\sqrt{2 \pi \sigma^{2}}} \int_{0}^{P G_{\text {sched }}}\left(P G_{\text {sched }}-\overline{\overline{P G}}\right) e^{-\frac{(P G-\overline{\overline{P G}})^{2}}{2 \sigma^{2}}} d P G \\
& \text { EENS }_{G}=\frac{P G_{\text {sched }}}{\sqrt{2 \pi \sigma^{2}}} \int_{0}^{P G_{\text {sched }}} e^{-\frac{(P G-\overline{\overline{P G}})^{2}}{2 \sigma^{2}}} d P G- \\
& \frac{1}{\sqrt{2 \pi \sigma^{2}}} \int_{0}^{P G_{\text {sched }}} P G \cdot e^{-\frac{(P G-\overline{\overline{P G}})^{2}}{2 \sigma^{2}}} d P G
\end{aligned}
$$

Solving equation (2.2.4.1):

$$
\frac{P G_{\text {sched }}}{2}\left[1+\operatorname{erf} \frac{(P G-\overline{\overline{P G}})}{2 \sigma^{2}}\right]
$$

Where

$$
\operatorname{erf}(x)=\frac{2}{\sqrt{\pi}} \int_{0}^{x} e^{-t} d x
$$

Solving equation (2.2.4.2) with integration by parts: 


$$
\begin{aligned}
& \text { let } a=\frac{1}{2 \sigma^{2}} \text {, and } c=\frac{1}{\sqrt{2 \pi \sigma^{2}}} \\
& u=c e^{-a \overline{\overline{P G^{2}}}} e^{-a P G^{2}}, \quad d u=2 a c \overline{\overline{P G}} e^{-a \overline{\overline{P G^{2}}}} \\
& d v=x e^{-a P G^{2}}, \quad v=-\frac{1}{2 a} e^{-a P G^{2}} \\
& (2.2 .4 .2)=u v-\int v \cdot d v \\
& \text { (2.2.4.2) }=-c e^{-a\left(P G_{\text {sched }}-\overline{\overline{P G}}\right)^{2}}-\overline{\overline{P G}} \int_{0}^{P G_{\text {sched }}} e^{-a P G^{2}} e^{2 a \overline{\overline{P G P G}}} e^{-a \overline{\overline{P G^{2}}}} d P G \\
& \text { (2.2.4.2) }=-c e^{-a\left(P G_{\text {sched }}-\overline{\overline{P G}}\right)^{2}}+a \overline{\overline{P G}}\left[1+\operatorname{erf}\left(a P G_{\text {sched }}-\overline{\overline{P G}}\right)\right]
\end{aligned}
$$

Thus, EENS is given by:

$$
\begin{aligned}
& \operatorname{EENS}_{G}=\frac{1}{\sqrt{2 \pi \sigma^{2}}} \frac{e^{-\left(P G_{\text {sched }}-\overline{\overline{P G}}\right)^{2}}}{2 \sigma^{2}}+\left(\frac{P G_{\text {sched }}}{2}-\frac{\overline{\overline{P G}}}{2 \sigma^{2}}\right) \\
& \operatorname{erf}\left(\frac{P G_{\text {sched }}-\overline{\overline{P G}}}{2} \sigma^{2}\right)+\frac{P G_{\text {sched }}}{2}-\frac{\overline{\overline{P G}}}{2} \sigma^{2}
\end{aligned}
$$

Here, $\overline{\overline{P G}}$, and $\sigma^{2}$ are given by equations (2.2.3.2), and (2.2.3.3), respectively.

A closed-form solution does not exist for the Gaussian because of the presence of the error function in the formulation as seen in equation (2.2.1.1). However, a number of closedform approximations of the error function exist and a suitable one can be utilized. Numerical methods and look-up tables are also available.

Cauchy: A solution for EENS calculated with a Cauchy distribution at a given $P G_{\text {sched }}$ is derived below: 


$$
\begin{aligned}
& \text { EENS }_{C}=\int_{0}^{P G_{\text {sched }}}\left(P G_{\text {sched }}-P G\right) \frac{1}{\pi \gamma\left[1+\frac{P G-\overline{\overline{P G}}}{\gamma}\right]} d P G \\
& \text { EENS }_{C}=\frac{P G_{\text {sched }}}{\pi \gamma} \int_{0}^{P G_{\text {sched }}} \frac{1}{1+\frac{P G-\overline{\overline{P G}}}{\gamma}} d P G- \\
& \frac{1}{\sqrt{2 \pi \sigma^{2}}} \int_{0}^{P G_{\text {sched }}} \frac{P G}{1+\frac{P G-\overline{\overline{P G}}}{\gamma}} d P G
\end{aligned}
$$

Solving equation (2.2.4.6):

(2.2.4.6) $=P G_{\text {sched }}\left[\frac{1}{\pi} \tan ^{-1}\left(\frac{P G-\overline{\overline{P G}}}{\gamma}\right)+\frac{1}{2}\right]$

Solving equation (2.2.4.7) with integration by parts:

Finding the integration constant $C$. At $P G_{\text {sched }}=0, E E N S=0$.

$$
\begin{aligned}
& 0=-\frac{\overline{\overline{P G}}}{\pi} \tan ^{-1}\left(-\frac{\overline{\overline{P G}}}{\gamma}\right)-\ln \left|\overline{\overline{P G^{2}}}+\gamma^{2}\right|-C \\
& C=-\frac{\overline{\overline{P G}}}{\pi} \tan ^{-1}\left(-\frac{\overline{\overline{P G}}}{\gamma}\right)-\ln \left|\overline{\overline{P G^{2}}}+\gamma^{2}\right|
\end{aligned}
$$

Thus, EENS is given by:

$$
\begin{aligned}
& E_{C}=\frac{P G_{\text {sched }}-\overline{\overline{P G}}}{\pi} \tan ^{-1}\left(P G_{\text {sched }}-\overline{\overline{P G}}\right)-\frac{\gamma}{2 \pi} \ln \left|\left(\frac{P G_{\text {sched }}-\overline{\overline{P G}}}{\gamma}\right)^{2}\right|+ \\
& \frac{P G_{\text {sched }}}{2}+\frac{\overline{\overline{P G}}}{\pi} \tan ^{-1}\left(\frac{-\overline{\overline{P G}}}{\gamma}\right)+\ln \left|\overline{\overline{P G^{2}}}\right|+\gamma^{2}
\end{aligned}
$$


Here, $\overline{\overline{P G}}$, is again given by equation (2.2.3.2). $\gamma$ is given by equation (2.2.3.5). It should be noted that EENS is a closed-form equation when using the Cauchy distribution. Equations (2.2.4.5) and (2.2.4.10) form two of the major contributions of this work. The closed form solutions (pseudo-closed form in the case of Gaussian) for EENS provide an accurate and easily calculable estimate for EENS that did not previously exist.

\subsection{Markov Chain Model}

\subsubsection{Theoretical Background}

A Markov chain is a stochastic process with the Markov property on a finite or countable state space, that is, if the conditional probability distribution of future states depends only on the current state. Thus, future states of a Markov chain do not depend on past states.

The state variable for power generation is divided into $N$ bins, equally spaced intervals from 0 to $P G_{\text {nominal }}$ of length $P G_{\text {nominal }} / N$. These states are defined as [5]:

$$
S=\left\{S_{1}, S_{2} \ldots S_{N}\right\}
$$

Every state $S i$ corresponds to an amount of power generation which is defined as:

$$
S_{i}=\left(\frac{i \cdot P G_{n o \min a l}}{N}\right)+\frac{P G_{n o \min a l}}{2 N}
$$

The parameter $N$ gives the size of the bins and is selected to accommodate the tradeoffs between precision and noise from sampling randomness. The $P G_{n o m i n a l} / 2 N$ term results from the fact that each state $S_{i}$ is chosen to be the middle of the bin. 
Changes from time $t$ to $t+1$ are defined by a one-step transition probabilities matrix $\mathbf{P}$. $\mathbf{P}$ is a symmetrical $N \mathrm{x} N$ matrix.

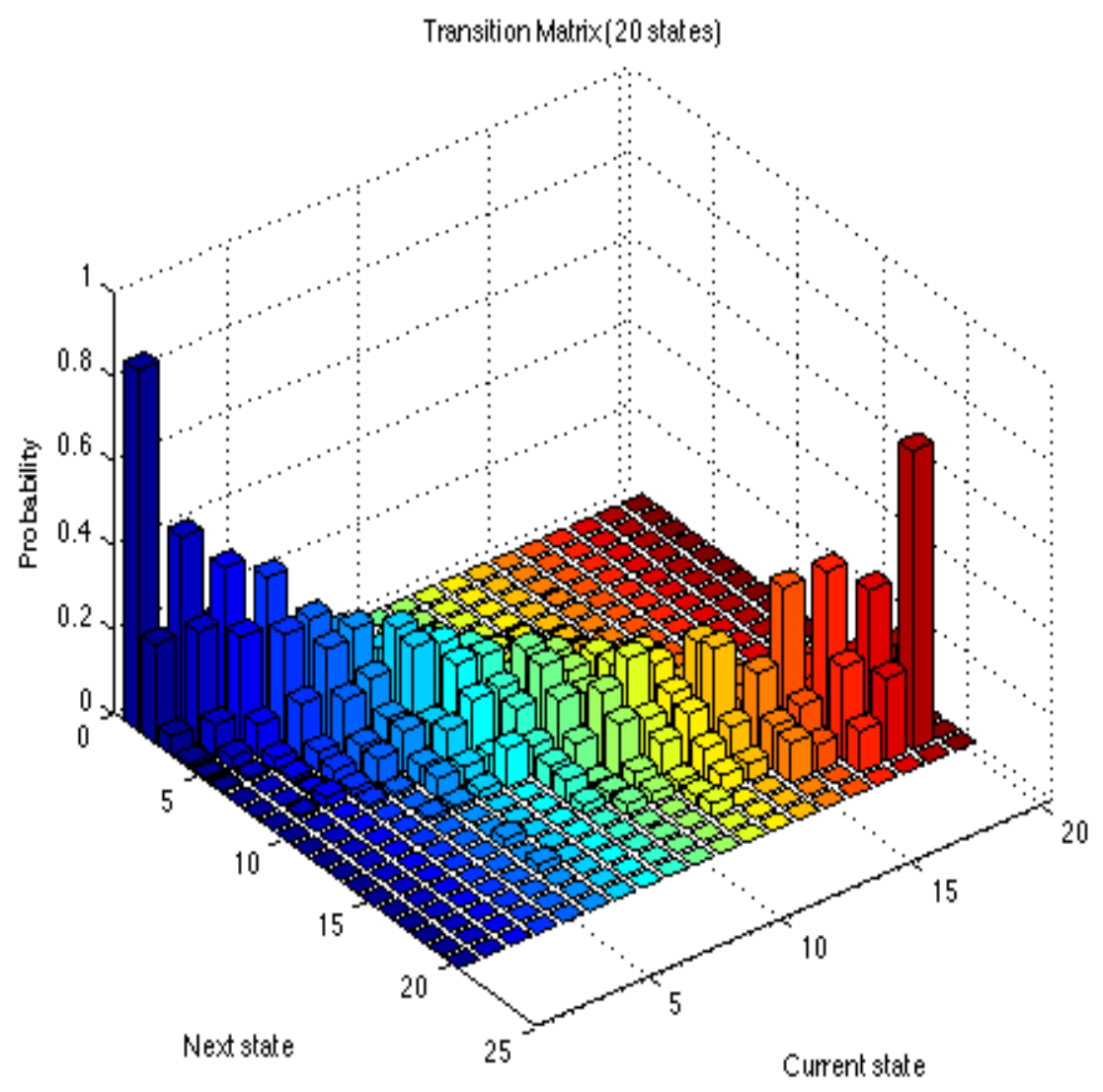

Figure 2.3.1.1: Example of a transition matrix created by a Markov chain model with $N=20$

Figure 2.3.1.1 shows the highest probability clusters are centred around $N=0$, and $N=$ 20. This agrees with the assumption made previously that the WEG is most often operating at 0MW or at full power $\left(P G_{\text {nominal }}\right)$. The above figure also shows that most transitions are centred around a diagonal from $(0,0)$ to $(\mathrm{N}, \mathrm{N})$ which agrees with the assumption that WEG generation does not change very much hour to hour and the most probable transition is generally to the same state. 
The estimate of the transition probabilities matrix $\mathbf{P}$ is determined based on the historical data and denoted as $\hat{\boldsymbol{P}}$. The columns of $\hat{\boldsymbol{P}}$ correspond to the current state of the process and the rows correspond to the state at the next time step. The generic element of $\hat{\boldsymbol{P}}$ is defined as $p_{i j}(t)$ and the generic row of $\hat{\boldsymbol{P}}$ is defined as $P_{i}(t)$ at time $t$. Each element of the transition matrix $\hat{\boldsymbol{P}}$ corresponds to a possible state of the process. Figure 3 shows an example of a transition matrix $\hat{\boldsymbol{P}}$ with 20 states.

An estimate for $p_{i j}(t)$ is given in [5] and shown below:

$$
\hat{p}_{i j}=\frac{n_{i j}(t)}{\sum_{j} n_{i j}(t)} \forall i, j, \sum_{j=1}^{N} \hat{p}_{i j}(t)=1 \forall i
$$

$n i j$ is the number of transitions from state $S_{i}$ to state $S_{j}$ observed in the training data series.

Thus, $P_{i}$ at time $t$ can be defined as:

$$
P_{i}(t)=\left\{\hat{p}_{i 1}(t), \hat{p}_{i 2}(t) \ldots \hat{p}_{i N}(t)\right\}
$$




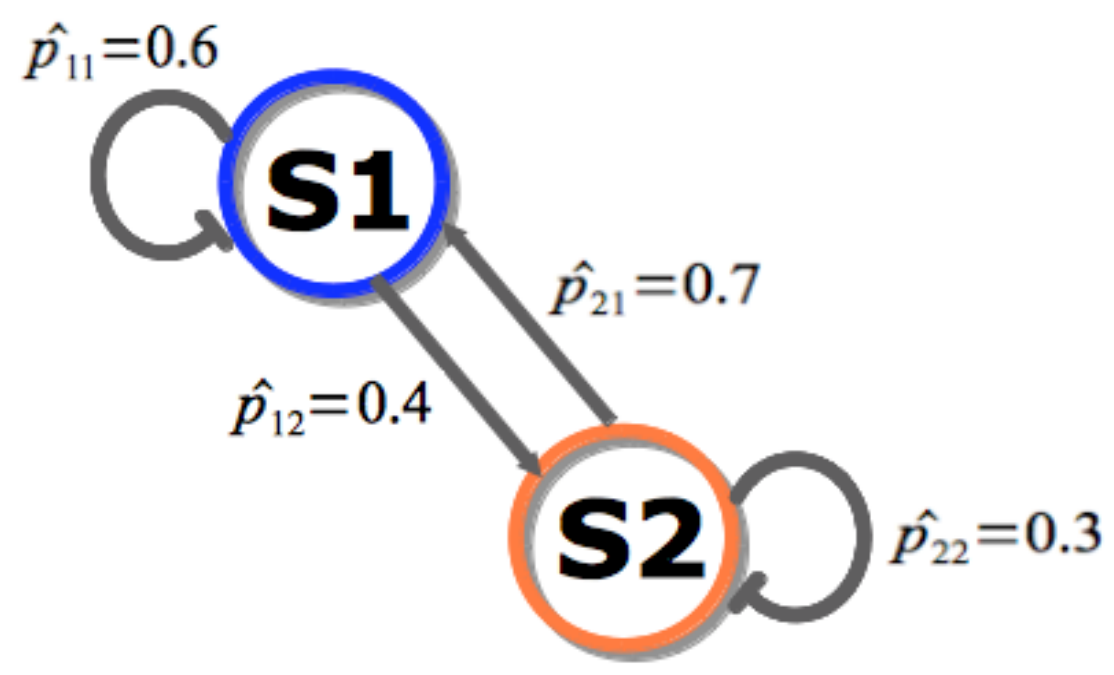

Figure 2.3.1.2: Sample two state Markov chain model

Figure 2.3.1.2 shows a simple Markov chain with two states. The numbers indicate the probability that the given state transition will occur. Note that they add up to 1 for each state. It can also be observed that a Markov chain with one state is equivalent to the persistence model as seen from equation (2.1.1.1) since every state transition is from the current state to itself.

Given that the generation at time $t$ corresponds to state $S_{i}$, each element of row $P_{i}(t)$ of the estimated transition matrix $\hat{\boldsymbol{P}}$ gives the probability of the state of the next time step. Thus, $P_{i}(t)$ is a discrete probability distribution of the WEG output at the next time step. A point forecast can be obtained by using the state with the highest probability given by the previous time step. At time $t$, the largest element of $P_{i}(t)$ gives the most probable state at the next time step which is defined as $S_{\max }(t)$.

$$
S_{\max }(t)=\underset{i}{M A X} \hat{P}_{i j}(t-1)
$$


In the case where multiple states are equally likely, $S_{\max }(t)$ is the average of those states. $S_{\max }(t)$ corresponds to the forecasted WEG generation at time $\hat{X}_{t}$ which is expressed in MW. Thus, $\hat{X}_{t}$ is given by:

$$
\hat{X}_{t}=N \cdot S_{\max }(t)+\frac{P G_{\text {nominal }}}{2 N}
$$

\subsubsection{Methodology}

Equation (2.3.1.3) is applied to all elements of the transition matrix $\hat{\boldsymbol{P}}$. The result can be visualized by Figure 3. The point forecast of time $t$ is given by equation (2.3.1.6).

\subsubsection{Probability Density Function}

As discussed previously, the Markov chain model differs from the persistence model in that the forecasted generation $P G$ is given not as a point forecast but rather as a probability distribution. Therefore, in the case of the Markov chain model, the probability density function of the forecast does not need to be fit to a Gaussian nor a Cauchy distribution.

Given the state is $S_{i}$ at time $t-1$, The vector $P_{i}(t)$ of the estimated transition matrix $\hat{\boldsymbol{P}}$ can immediately be used in EENS calculation. The PDF is thus given by: 


$$
P D F_{M}\left(P G_{t}\right)=P_{i}(t-1)
$$

\subsubsection{Calculating EENS}

The probability distribution given in the Markov chain forecast is the probability density function of the forecast error used to calculate EENS. This is a discrete probability density function and at time $t$ when the current generation is in state $S_{i}$, is given by $P_{i}(t)$. equation (2.1.1) can be reformulated as:

$$
E E N S_{M}=\sum_{k=1}^{P G_{\text {sched }}} p_{i k}(t) \cdot\left(P G_{\text {sched }}-P G\right) \cdot \frac{P_{n o \text { min } a l}}{N}
$$

\subsection{ARMA Model}

\subsubsection{Theoretical Background}

An autoregressive-moving-average (ARMA) model is a tool used for the analysis of time series composed of two polynomials and is used for modeling and predicting future values of both deterministic and stochastic processes. It is assumed that WEG generation exhibits short memory since WEG generation tends not to change very much over short time scales.

An ARMA model is composed of two parts: an auto-regressive (AR) part, and a movingaverage (MA) part. An $\operatorname{ARMA}(p, q)$ process is defined as an ARMA model where the AR portion has an order of $p$ and the MA portion has an order of $q$. The model is characterized as such [6]:

$$
\hat{X}_{t}=\sum_{j=1}^{p} a_{j} X_{t-j}+\sum_{k=0}^{q} b_{k} e_{t-k}
$$


The first term in equation (2.4.1.1) represents the auto-regressive component and the second term represents the moving average component. It shows that $\hat{X}_{t}$ is a linear combination of $p$ past observations at time $t$ (auto-regressive part) and a white-noise process with a mean of zero and a constant variance (moving-average part).

The simplest $\operatorname{ARMA}(p, q)$ process is one where $p$ is 1 and $q$ is 0 . Similarly to the Markov chain model, this reduces to the persistence model in equation (2.2.1.1) since the model only uses the WEG generation of the previous time step.

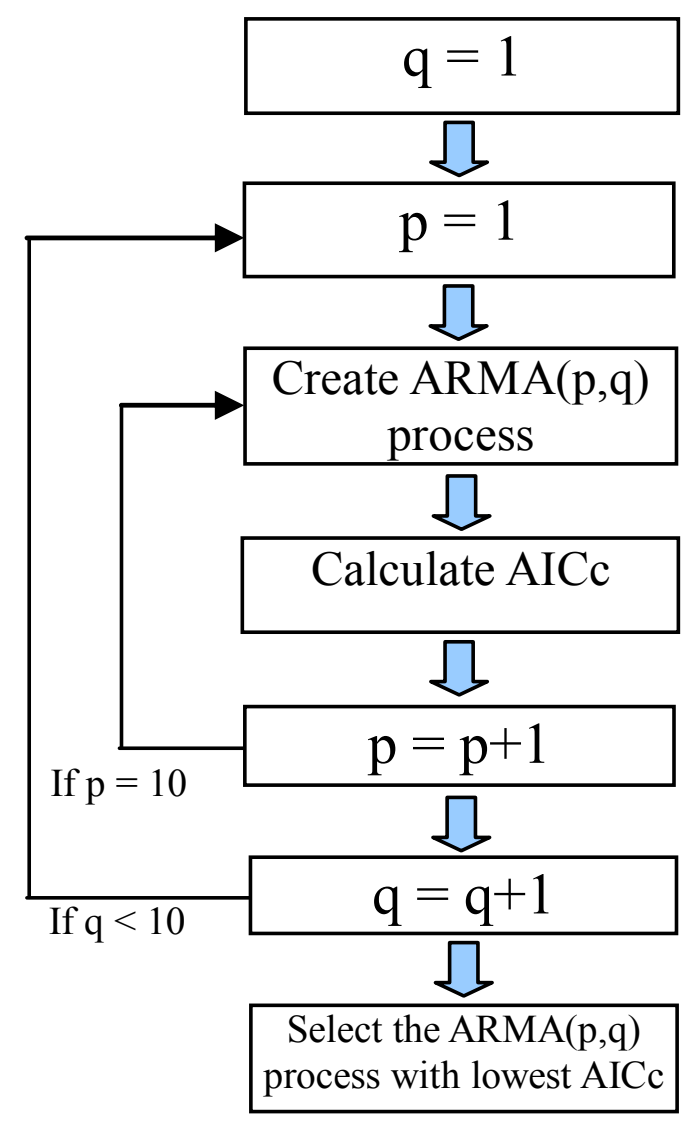

Figure 2.4.1.1: Flowchart depicting the process of determining 
$\hat{X}_{t}$ is an $\operatorname{ARMA}(p, q)$ process where $\mathrm{p}$, and $\mathrm{q}$ are the orders of the auto-regressive part and moving average parts respectively. Two tasks remain: the first is to select the orders $\mathrm{p}$, and $\mathrm{q}$ of the model and the second is to select the coefficients $a_{j}$ and $b_{k}$. Matlab's System Identification toolbox has tools that can be used for both tasks.

\subsubsection{Model Order}

The Akaike information criterion (AIC) is a tool used for model selection. It evaluates statistical models used for a given data set relative to each other. It compares the trade-off between the goodness of the fit and the number of parameters used in the model. One caveat of its use however is that because it only provides a relative measure, it is unable to tell if none of the models compared are good fits. AIC is given below[13].

$$
A I C=2 k-2 \ln (L)
$$

Here, $\mathrm{k}$ is the number of parameters in the statistical model and $L$ is the maximized value of the likelihood function of the estimated model which is assumed to be Gaussian and is given by equation (2.1.3.1). $\ln (L)$ is the $\log$-likelihood of the likelihood function $L$ and is given by:

$$
\ln (L)=\ln \left(\frac{1}{\sqrt{2 \pi \sigma_{2}}}\right)-\left(\frac{\overline{\overline{P G}}-P G}{2 \sigma_{2}}\right)
$$

$\overline{\overline{P G}}$ Is given by equation (2.2.3.2), and $\sigma^{2}$ is given by equation $(2.2 .3 .3)$.

The preferred model is the one with the lowest AIC. The first term in equation $(2.4 .2 .1)$ penalizes a model for over-fitting and the second term rewards the goodness of the fit.

AICc is a modification on AIC that corrects for finite sample sizes. It is given below[14]. 


$$
A I C c=A I C+\frac{2 k(k+1)}{n-k-1}
$$

AICc is similar to AIC but carries a greater penalty for models with extra parameters. AICc will be used as the main criterion for order selection in ARMA models.

\subsubsection{Coefficient Determination}

A number of methods can be used for determining the $p$ and $q$ coefficients of an ARMA process such as Burg and Shanks, and Yule-Walker, or least-squares estimation. Least-squares estimation is used.

\subsubsection{Methodology}

The first step is to determine the model order. An $\operatorname{ARMA}(\mathrm{p}, \mathrm{q})$ process is created with the training data as the input for each $\mathrm{p}, \mathrm{q}=\{1,2, \ldots 10\}$. Matlab's internal least-squares estimation method is used in determining $p$, and $q[15]$.Therefore, $100 \operatorname{ARMA}(p, q)$ processes will be created and AICc is calculated for each of them. The one with the lowest AICc is used to determine $p$ and q. Figure 4 below illustrates the method of model order selection.

Once the orders of the model have been selected, equation (2.4.1.1) can be used to predict each future time-step. This can be used to either model the training data or to predict future values. 


\subsubsection{Probability Density Function}

As noted previously, a probability distribution of a given point forecast's error is required for EENS calculation.

The method of producing a probability density function for the ARMA model forecast is the same as in persistence model and is described in section 2.2.3. The one difference in the case of ARMA model is that $\overline{\overline{P G_{t}}}$ is given by equation (2.4.1.1). $\sigma^{2}$ and $\gamma$ are given by equations 2.2.3.3 and 2.2.3.5 for Gaussian and Cauchy distributions, respectively. With these two sets of parameters, EENS can be calculated.

\subsubsection{Calculating EENS}

EENS for the ARMA model is calculated in the same manner as persistence model. Using the Gaussian distribution, EENS is given by equation 2.2.4.5 and by 2.2.4.10 when using the Cauchy distribution. Again, $\overline{\overline{P G_{t}}}$ is given by equation (2.4.1.1). 


\section{Results AND Discussion}

\subsection{Data}

Historical hourly power generation data for every wind generator/farm in Ontario was collected from the Independent Electricity System Operator (IESO) web-site from March 3, 2013 to October 2, 2013. In the models and calculations that follow, data from the Amaranth wind farm is used. Because WEGs often operate at maximum capacity or not at all, the nominal power generation $P G_{\text {nominal }}$ is defined as the maximum generation of the WEG.

Two sets of data are used: a training data set, and validation data set. The training data is used to construct the Markov chain and ARMA models (persistence does not require training) The validation data follows the training data immediately in time and is used to verify the accuracy of each model. The training data contains 165 days of data (3960 hours) and the validation data contains 35 days ( 840 hours).

Data from March 3, 2013 to August 27, 2013 is used as train-ing data and data from August 28, 2013 to October 2, 2013 is used for the validation data.

\subsection{Amaranth Wind Farm}

\subsubsection{Comparing Forecast Error}

The three models are trained on the hourly training data based on 3960 hours of the Amaranth Wind Farm's wind energy output with a nominal power output, $P G_{\text {nominal }}$, of $200 \mathrm{MW}$. 
Each of the three models is used to generate a one-hour-ahead forecast for the duration of the validation data ( 840 hours). They are plotted against the actual wind generation of the Amaranth Wind Farm during that time period.

In the Markov chain model used, $N$ is chosen to be 200. In the ARMA model used, $p$ and $q$ are chosen to be 2 . Plots of each predictive model between the $200^{\text {th }}$ and $300^{\text {th }}$ hours of the validation data are shown below.

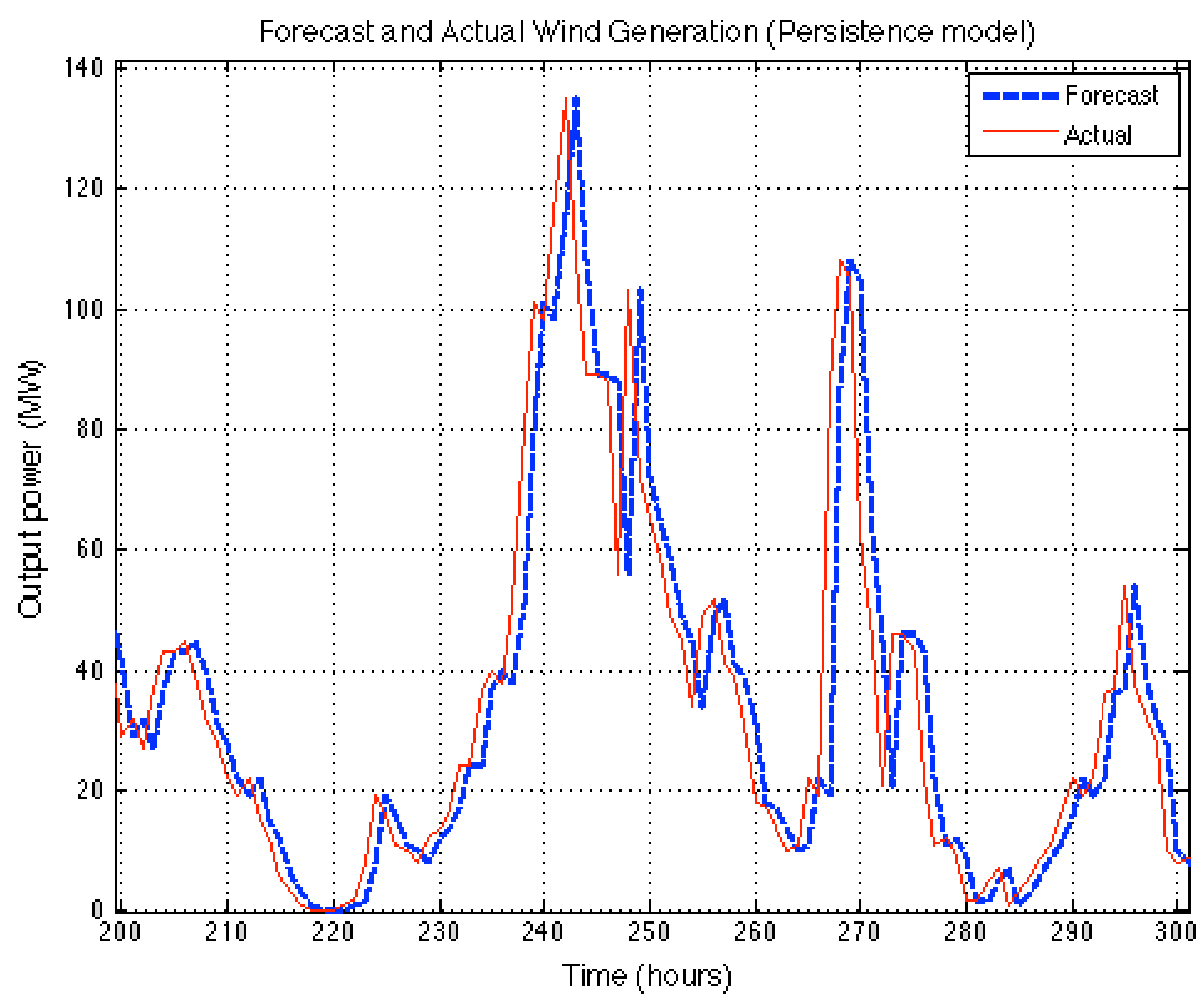

Figure 3.2.1.1: Persistence model wind energy generation forecast compared to actual generation for Amaranth wind farm 


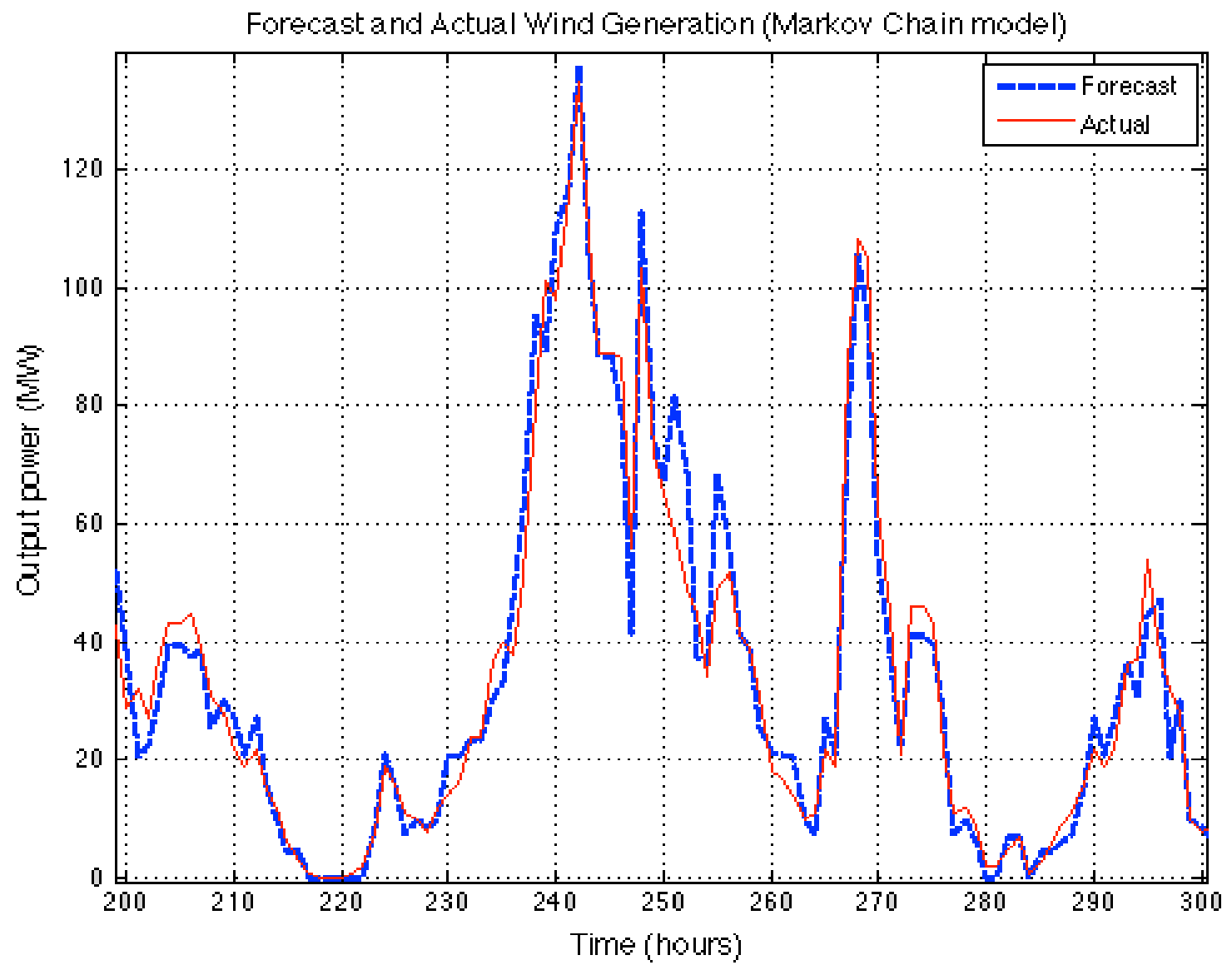

Figure 3.2.1.2: Markov chain model wind energy generation forecast compared to actual generation for Amaranth wind farm 


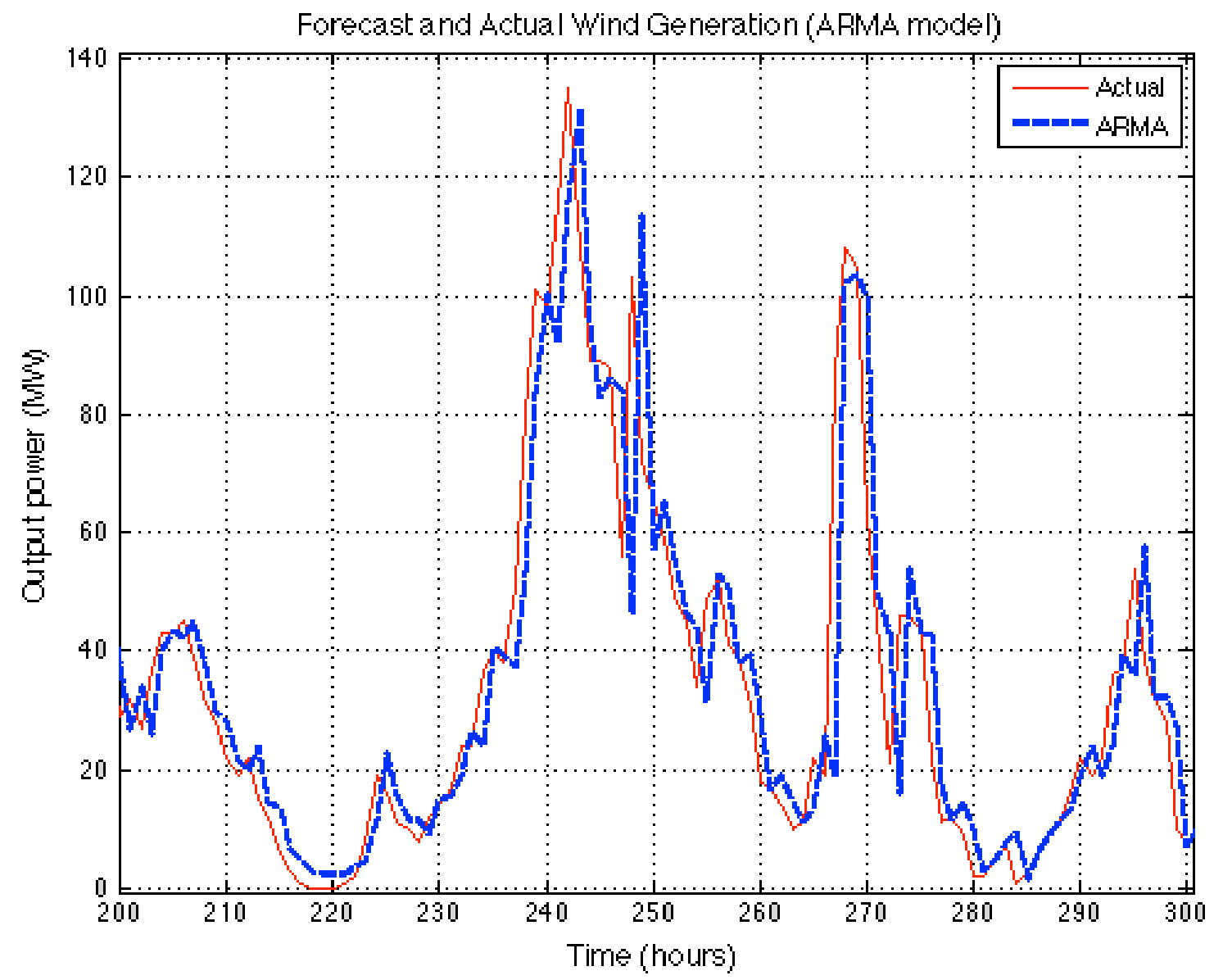

Figure 3.2.1.3: ARMA model wind energy generation forecast compared to actual generation for Amaranth wind farm

Figures 5 through 7 show that all three models are capable of producing an accurate forecast for the one-hour-ahead horizon. The persistence model produces a forecast that is delayed by one hour based on the actual generation. Qualitatively, they show that Markov chain and ARMA model provide a more accurate forecast of than persistence model. 
The forecasts generated by each model is evaluated quantitatively by comparing their normalized root mean square error (NRMSE) which is calculated using the difference between forecasted generation and the actual generation over the duration, $m$, of the validation data period. It is expressed as a percentage of nominal power output of the WEG. NRMSE is defined by:

$$
N R M S E=\frac{1}{P G_{\text {no } \min a l}} \sqrt{\frac{1}{m} \sum_{t=1}^{m}\left(X_{t}-\hat{X}_{t}\right)^{2}} \times 100 \%
$$

The size of the validation period, $m$, is 840 elements. The results are summarized in the table below:

Table 3.2.1.1: NRMSE of forecast for each predictive model (Amaranth)

\begin{tabular}{|l|l|}
\hline Model & NRMSE (\% of PGnominal) \\
\hline Persistence & 6.3302 \\
\hline Markov & 2.6622 \\
\hline ARMA & 5.2277 \\
\hline
\end{tabular}




\section{Forecasting Errors (Amaranth)}

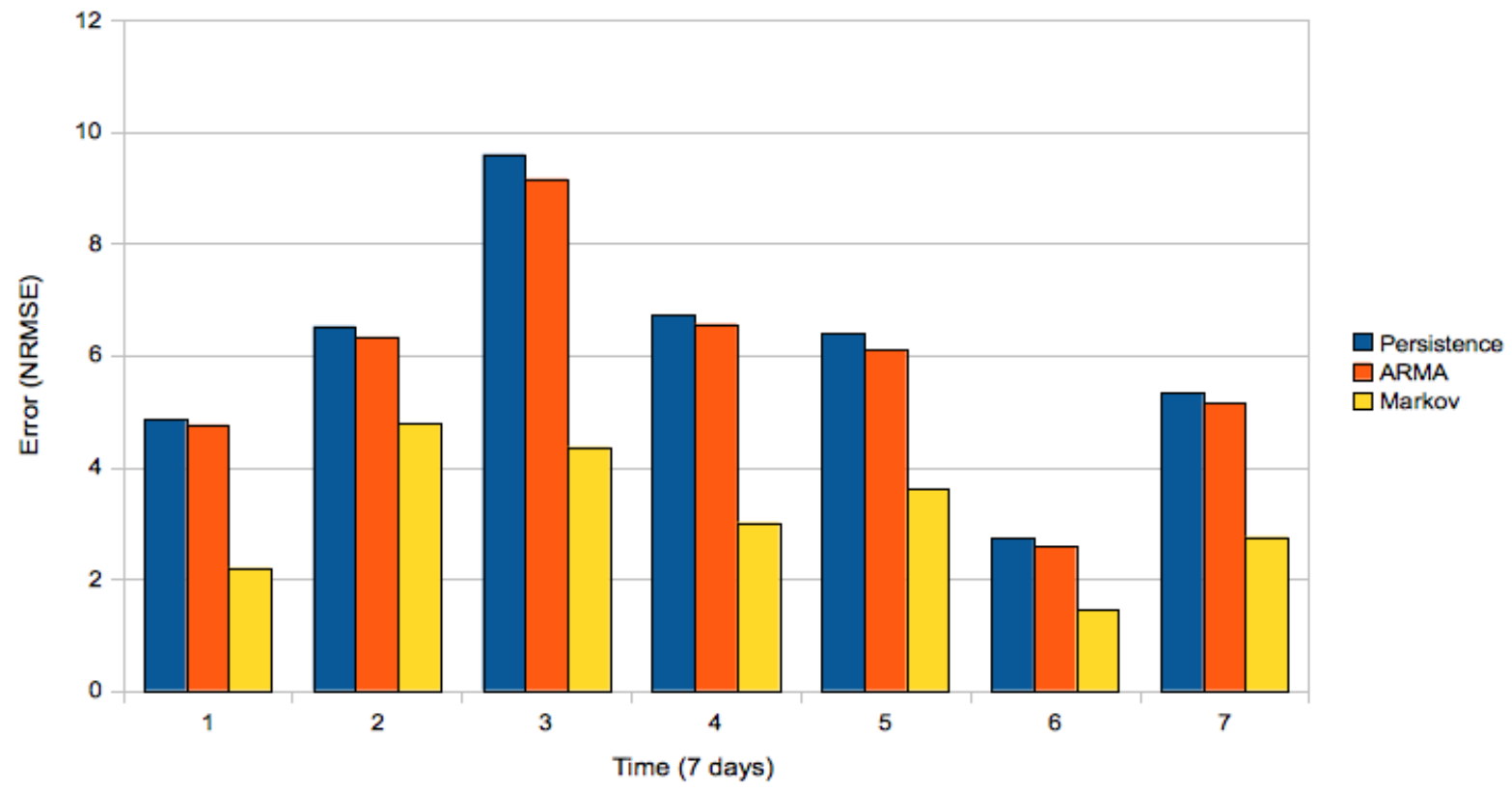

Figure 3.2.1.4: Forecast errors of all three predictive models plotted together for Amaranth wind farm 
Figure 8 shows the NRMSE of each predictive model side by side during windows of 168 hours (7 days). Both Markov chain and ARMA models performed better than the persistence model. Markov chain performed significantly better than persistence and ARMA. Markov chain should be expected to work best in cases where the training data provides an accurate assessment of the wind generation of the wind farm at all times.

\subsubsection{Comparing EENS}

EENS was calculated using the error distributions of persistence, Markov chain, and ARMA model forecasts on the one-hour-ahead horizon. Gaussian and Cauchy distributions were used for persistence and ARMA models and their effects on EENS compared. The distribution generated by the Markov chain model's forecast was used for EENS calculation. EENS is also compared to AENS in order to evaluate the adequacy of the calculated EENS. 


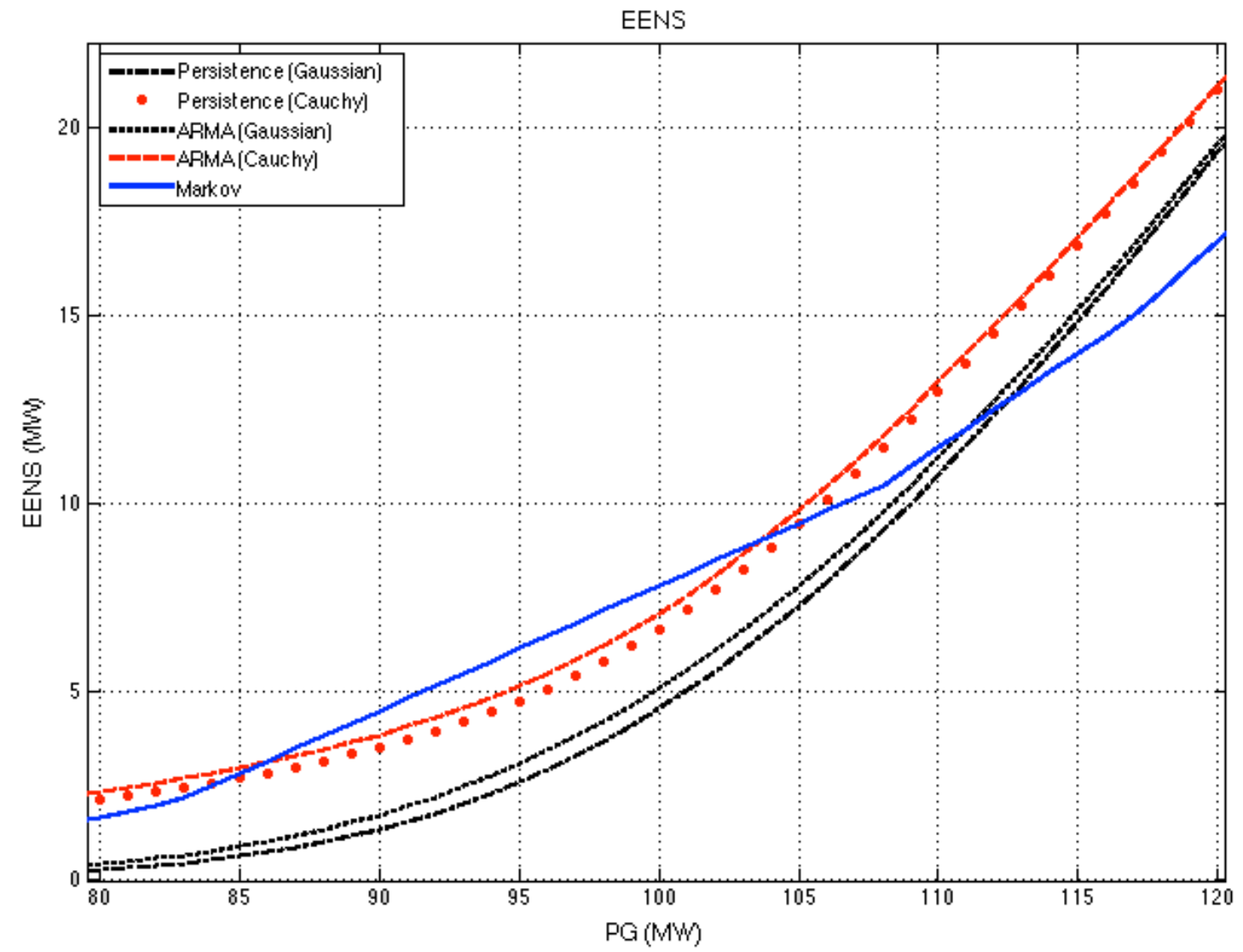

Figure 3.2.2.1: EENS calculated from persistence, Markov Chain, and ARMA models for Amaranth wind farm

The plot in figure 3.2.2.1 uses a forecasted generation $\overline{\overline{P G}}$, of $100 \mathrm{MW}$. It compares EENS estimations for the three models with Gaussian and Cauchy distributions used for persistence and ARMA. The scheduled generation $P G_{\text {sched }}$ is not necessarily known at the time of the forecast. For this reason, EENS is shown here as a function of $P G_{\text {sched. }}$ The X-axis of the plot gives the EENS for a given $P G_{\text {sched. }}$ 
In all cases at this forecasted generation, EENS is very small when the expected generation $P G_{\text {sched }}$ is less than $80 \mathrm{MW}$. This is explained by the fact that if the forecasted generation is at $100 \mathrm{MW}$, and $P G_{\text {sched }}$ is much less than $100 \mathrm{MW}$, there is a high probability that the expected generation will be met. Thus, EENS will be small. As $P G_{\text {sched }}$ increases, EENS increases as well.

Figure 3.2.2.1 shows that ARMA model using Gaussian distribution gives the smallest EENS and ARMA model using Cauchy distribution gives the greatest EENS. This difference is explained by the shorter tail of the Cauchy distribution relative to the Gaussian distribution. Markov Chain model gives the most moderate EENS estimation of the five scenarios shown in Figure 3.2.2.1. 


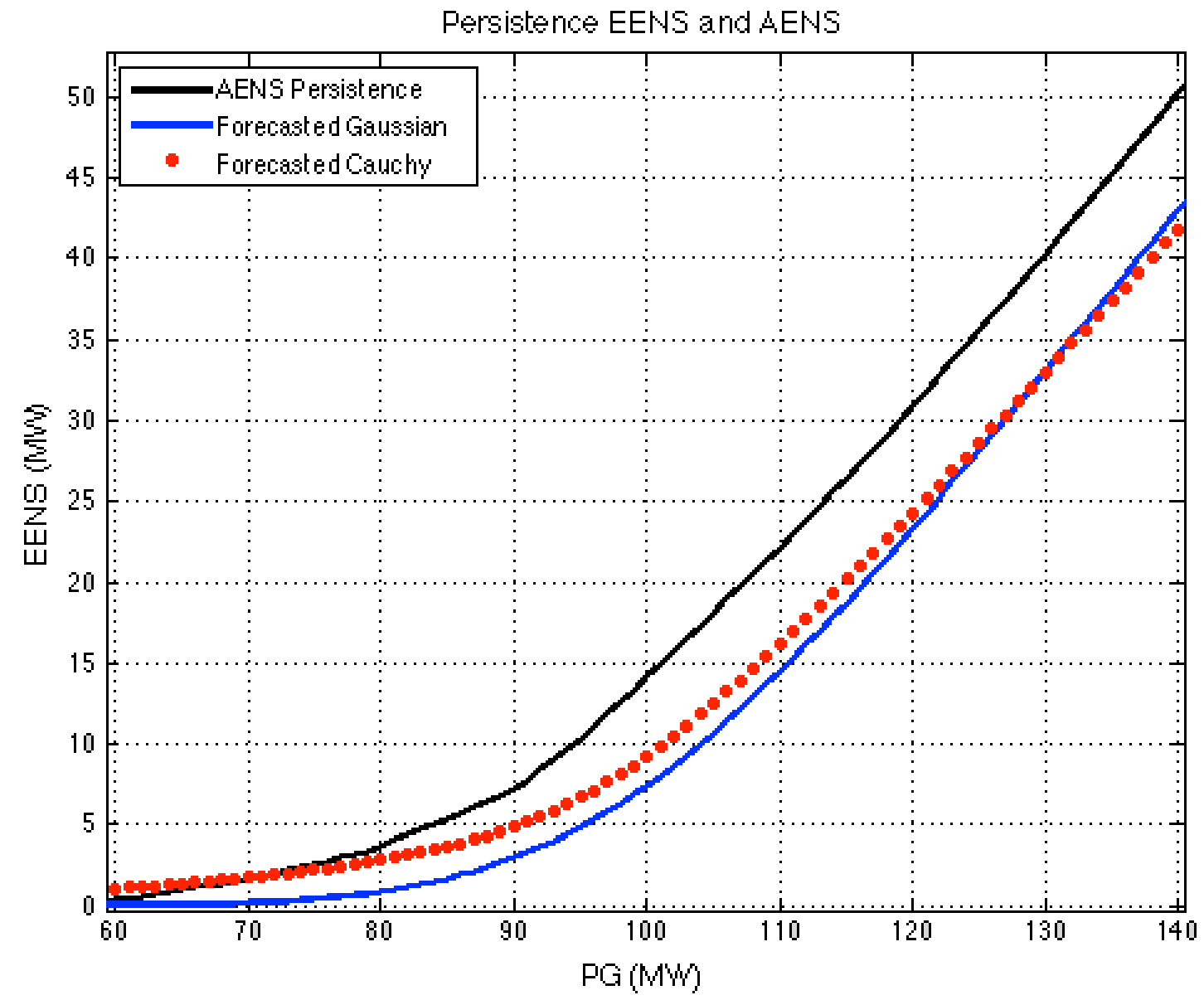

Figure 3.2.2.2: EENS plotted with AENS for persistence model with $\overline{\overline{P G}}$ at 96MW for Amaranth Island wind farm.

Figure 3.2.2.2 shows EENS plotted with actual energy not served (AENS) for persistence model using Gaussian and Cauchy distributions with $\overline{\overline{P G}}$ at $96 \mathrm{MW}$. AENS is the actual energy shortfall for a given amount of $P G_{\text {sched. }}$ AENS in figure 3.2.2.2 is determined as such:

1. Find all instances in the training data where $96 \mathrm{MW}$ is forecasted by the model.

2. Calculate the AENS for the actual generation at that time for all possible $P G_{\text {sched }}$. 
3. Calculate the mean of each instance.

It can be seen from the plot in figure 3.2.2.2 that the Cauchy distribution provides a better estimate of EENS than the Gaussian distribution.

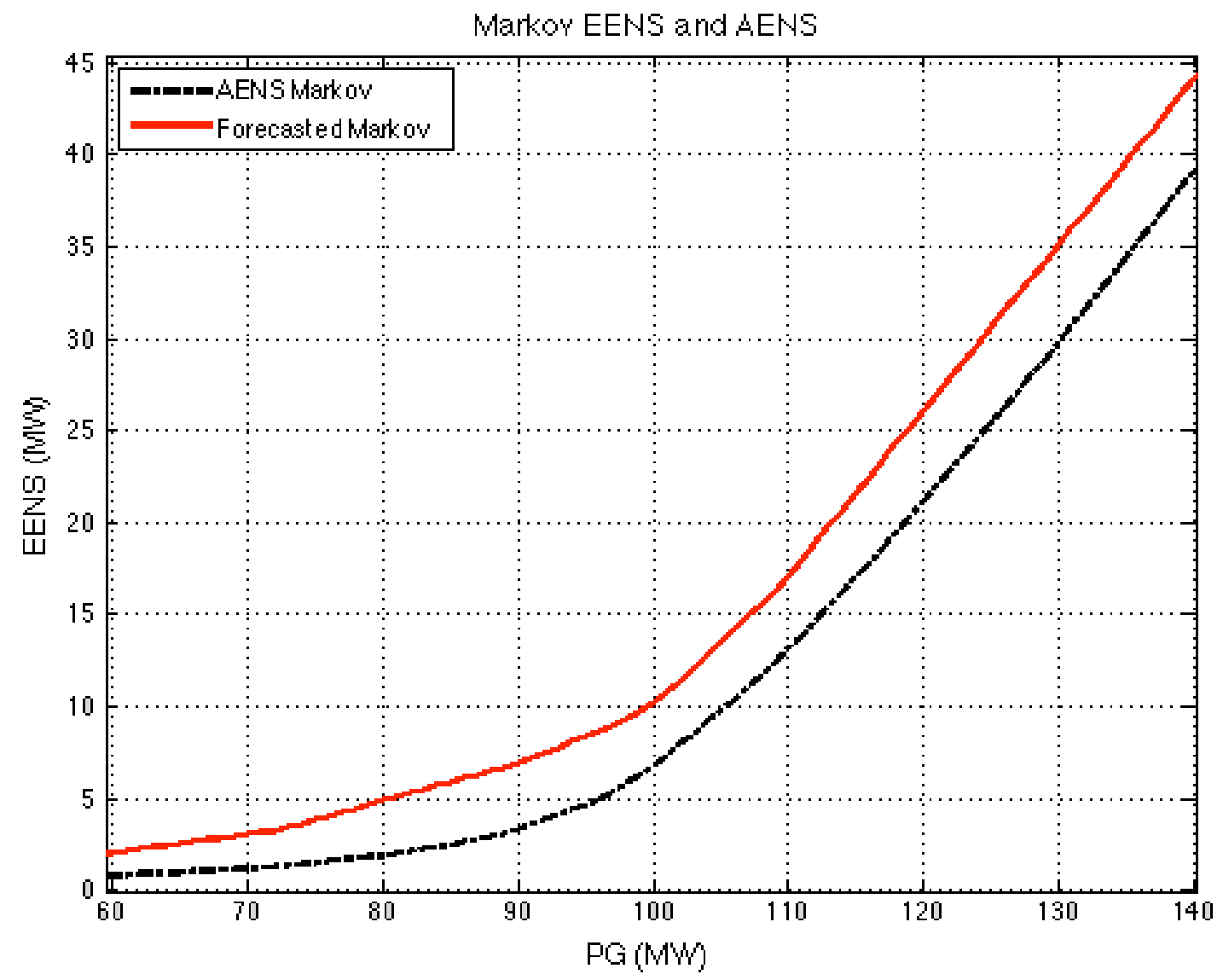

Figure 3.2.2.3: EENS plotted with AENS for Markov chain model with $\overline{\overline{P G}}$ at $96 M W$ for Amaranth wind farm. 
Figure 3.2.2.3 shows EENS plotted with AENS for Markov chain model with $\overline{\overline{P G}}$ at $96 \mathrm{MW}$. AENS in figure 3.2.2.3 is determined in the same way as in figure 3.2.2.2. The plot in figure 3.2.2.3 shows that Markov chain model provides a better estimate of EENS than the persistence model.

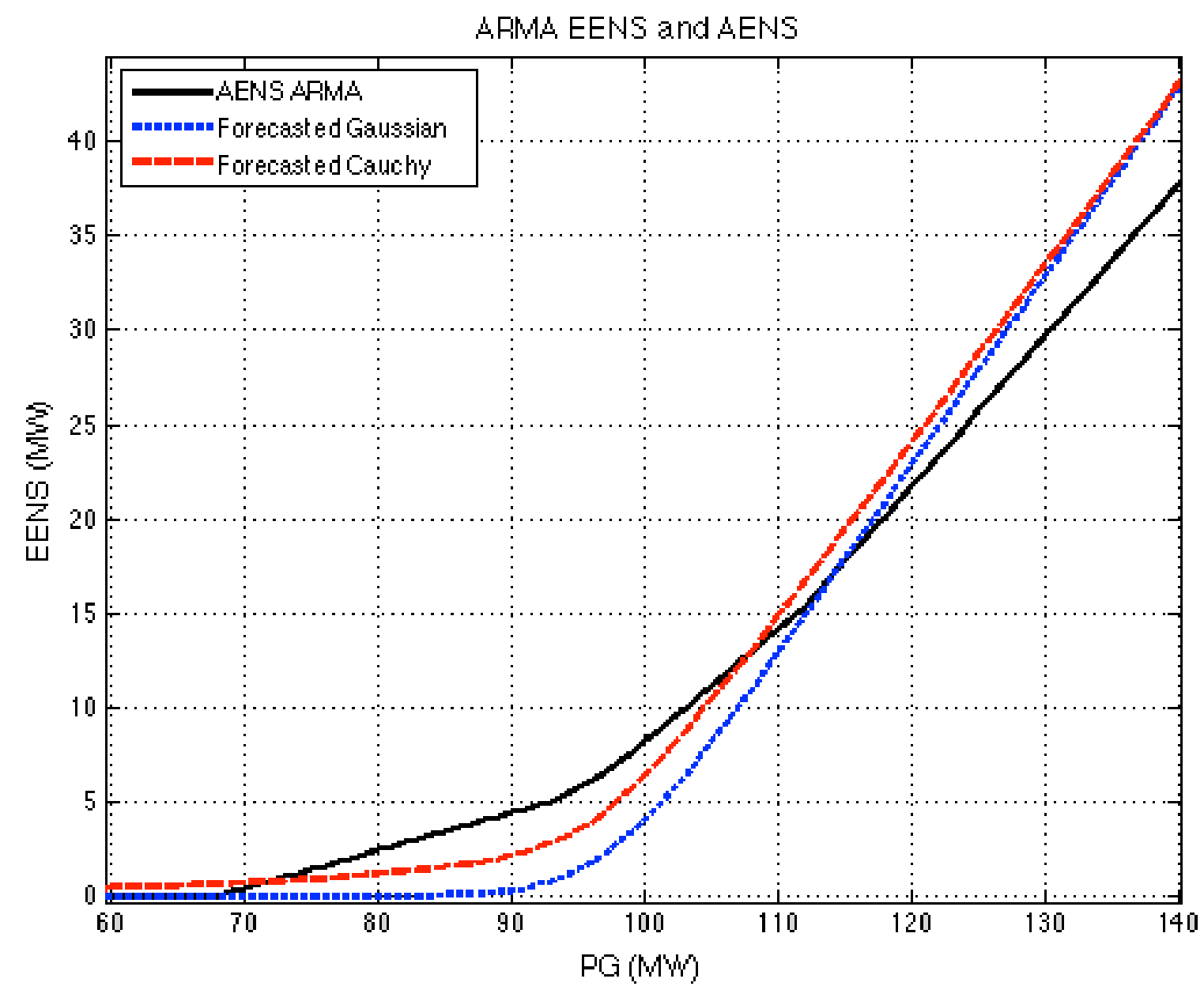

Figure 3.2.2.4: EENS plotted with AENS for ARMA model with $\overline{\overline{P G}}$ at $96 \mathrm{MW}$ for Amaranth wind farm. 
Figure 3.2.2.4 shows EENS plotted with AENS for ARMA model with $\overline{\overline{P G}}$ at $96 \mathrm{MW}$. AENS in figure 3.2.2.4 is determined in the same way as in figure 3.2.2.2. The plot in figure 3.2.2.4 shows that ARMA model provides a better estimate of EENS than the persistence model however it is difficult to determine qualitatively if it provides a better estimate than the Markov chain model.

Table 3.2.2.1: NRMSE of EENS vs. AENS for each predictive model and distribution (Amaranth)

\begin{tabular}{|l|l|}
\hline Model & NRMSE (\% of PGnominal) \\
\hline Persistence (Gaussian) & 9.91 \\
\hline Persistence (Cauchy) & 12.48 \\
\hline Markov Chain & 5.23 \\
\hline ARMA (Gaussian) & 12.41 \\
\hline ARMA (Cauchy) & 10.70 \\
\hline
\end{tabular}

Table 3.2.2.1 gives the NRMSE (as defined in section 3.2.1) between EENS and AENS for each model and distribution with $P G_{s c h e d}$. It can be seen that the Markov chain model provides the best estimate of the five scenarios but ARMA model using the Cauchy distribution also outperforms persistence model. In both persistence and ARMA models, Cauchy outperforms Gaussian. 


\subsection{Wolfe Island Wind Farm}

\subsubsection{Comparing Forecast Error}

The three models are trained on the hourly training data based on 4800 hours of the

Wolfe Island Wind Farm's wind energy output with a nominal power output, $P G_{\text {nominal }}$, of 197 MW.

Each of the three models is used to generate a one-hour-ahead forecast for the duration of the validation data ( 840 hours). They are plotted against the actual wind generation of the Wolfe Island Wind Farm during that time period.

In the Markov chain model used, $N$ is chosen to be 200. In the ARMA model used, $p$ and $q$ are chosen to be 2. Plots of each predictive model between the $200^{\text {th }}$ and $300^{\text {th }}$ hours of the validation data are shown below. 


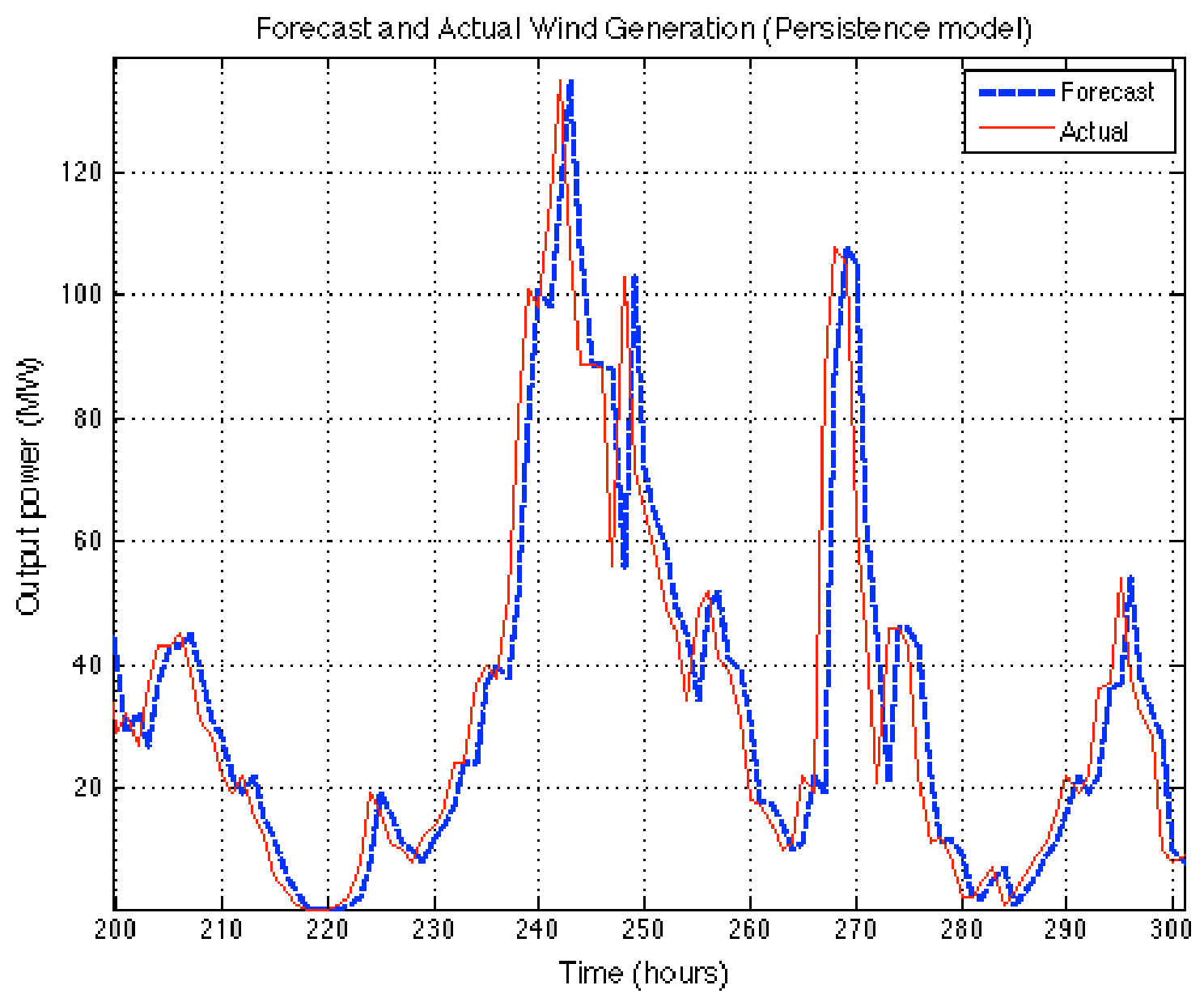

Figure 3.3.1.1: Persistence model wind energy generation forecast compared to actual generation for Wolfe Island wind farm 


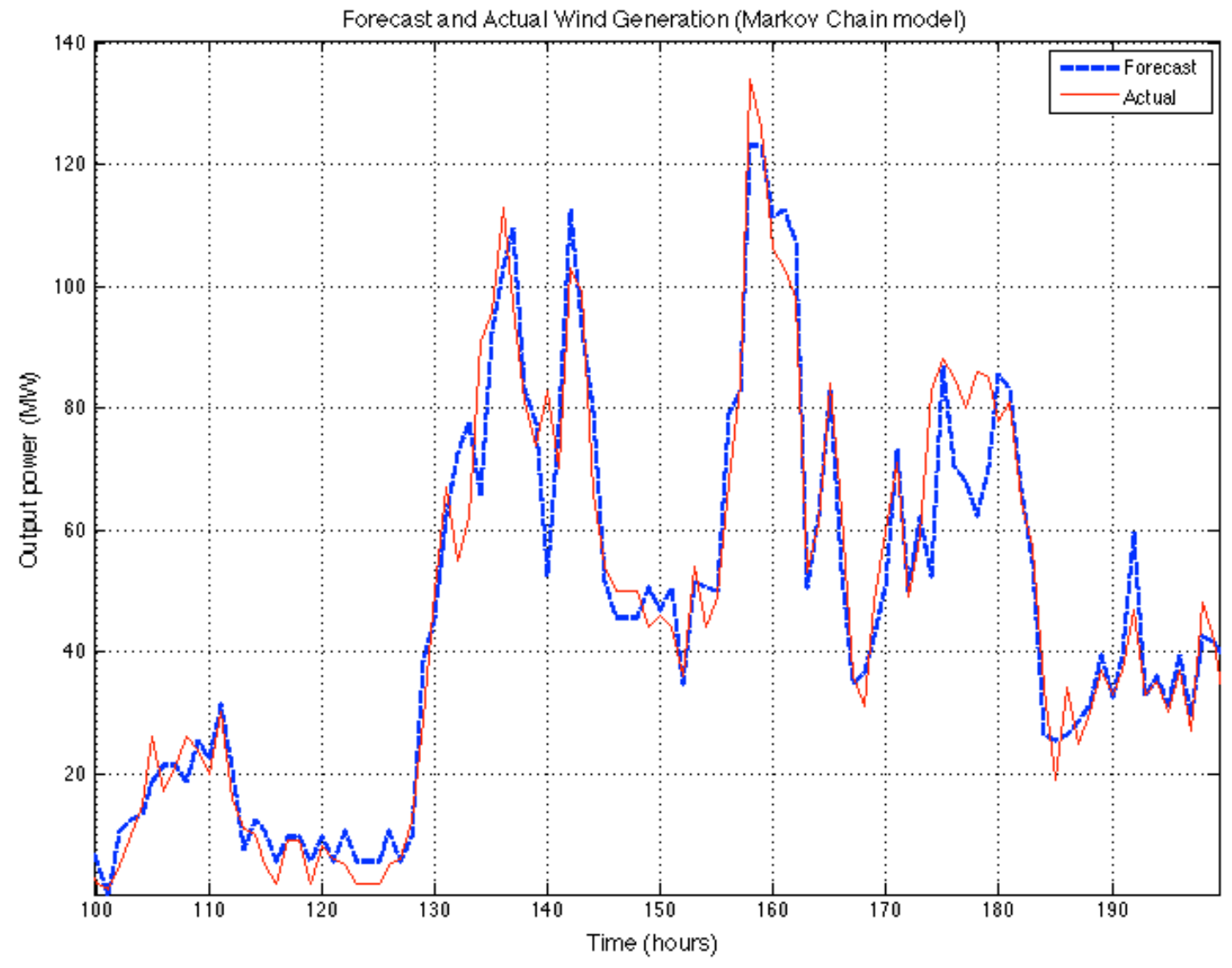

Figure 3.3.1.2: Persistence model wind energy generation forecast compared to actual generation for Wolfe Island wind farm 


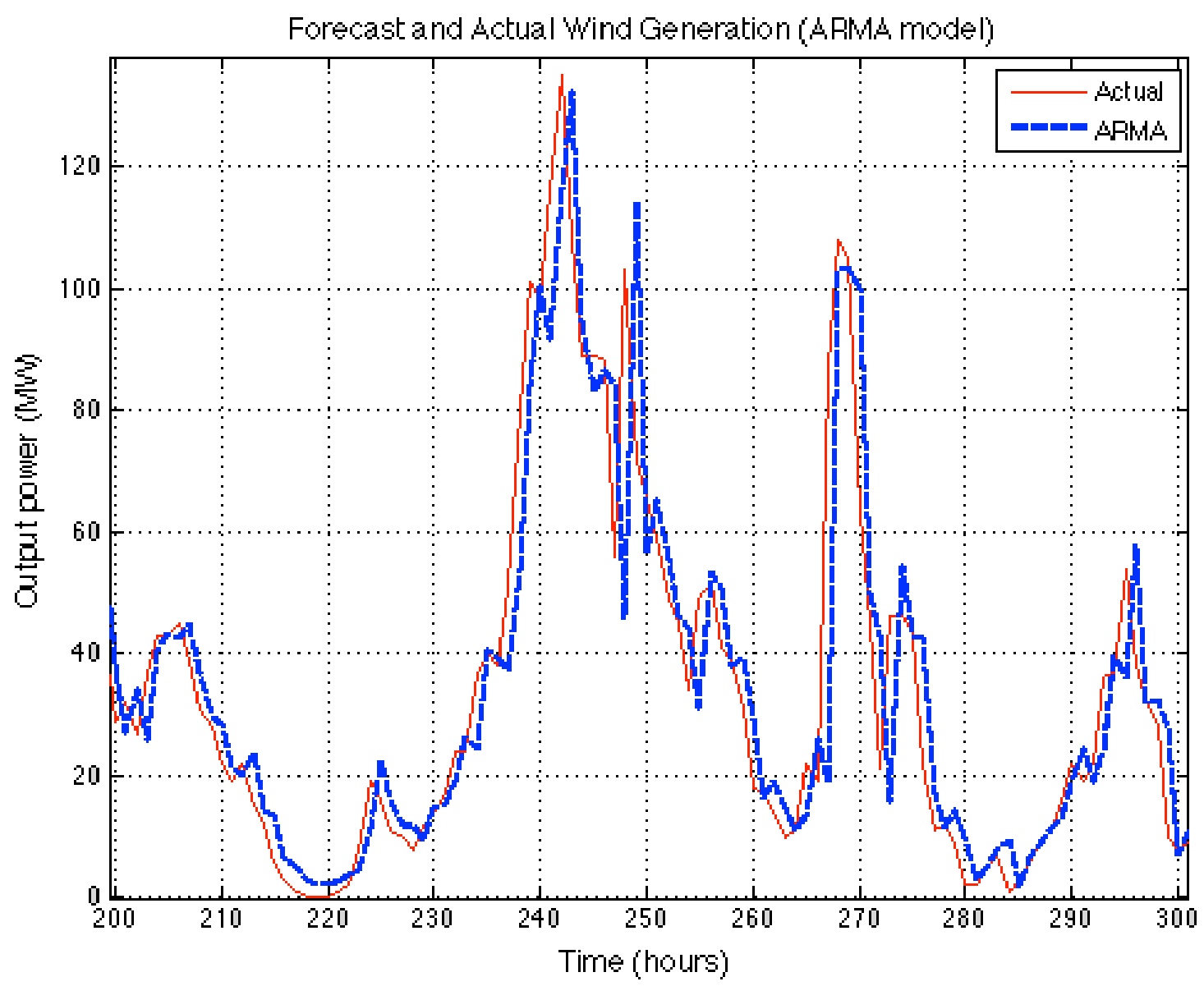

Figure 3.3.1.3: Persistence model wind energy generation forecast compared to actual generation for Wolfe Island wind farm 
Figures 10 through 12 show qualitatively that the three models provide good estimates of WEG generation for the Wolfe Island wind farm.

As in table 3.2.1.1, table 3.3.1.1 shows the NRMSE of each model and its predictive accuracy using the data from the Wolfe Island wind farm. The sample size, $m$, is 840 . The results are similar to the results from the Amaranth wind farm. The accuracy of each model is slightly worse in the case of Wolfe Island.

Table 3.3.1.1: NRMSE of forecast for each predictive model (Wolfe Island)

\begin{tabular}{|l|l|}
\hline Model & NRMSE (\% of $P G_{\text {nominal) }}$ \\
\hline Persistence & 6.4271 \\
\hline Markov & 2.8568 \\
\hline ARMA & 6.2634 \\
\hline
\end{tabular}




\section{Forecasting Errors (Wolfe Island)}

Time (days)

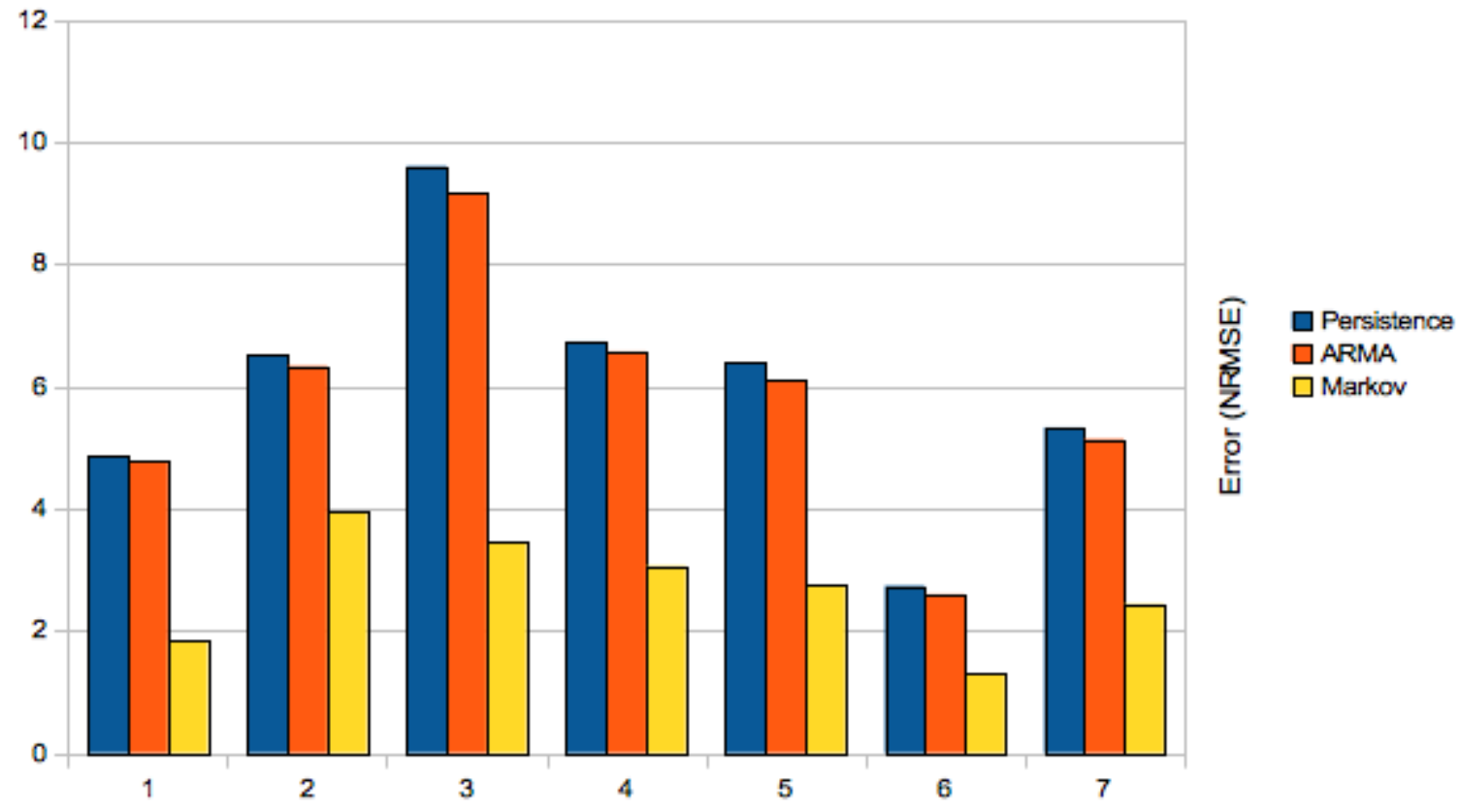

Figure 3.3.1.4: Forecast errors of all three predictive models plotted together for Wolfe Island wind farm

As in figure 3.2.1.4, figure 3.3.1.4 shows the NRMSE of each predictive model side by side during windows of 168 hours (7 days). Again, both Markov chain and ARMA models performed better than the persistence model and Markov chain performed the best of the three. 


\subsubsection{Comparing EENS}

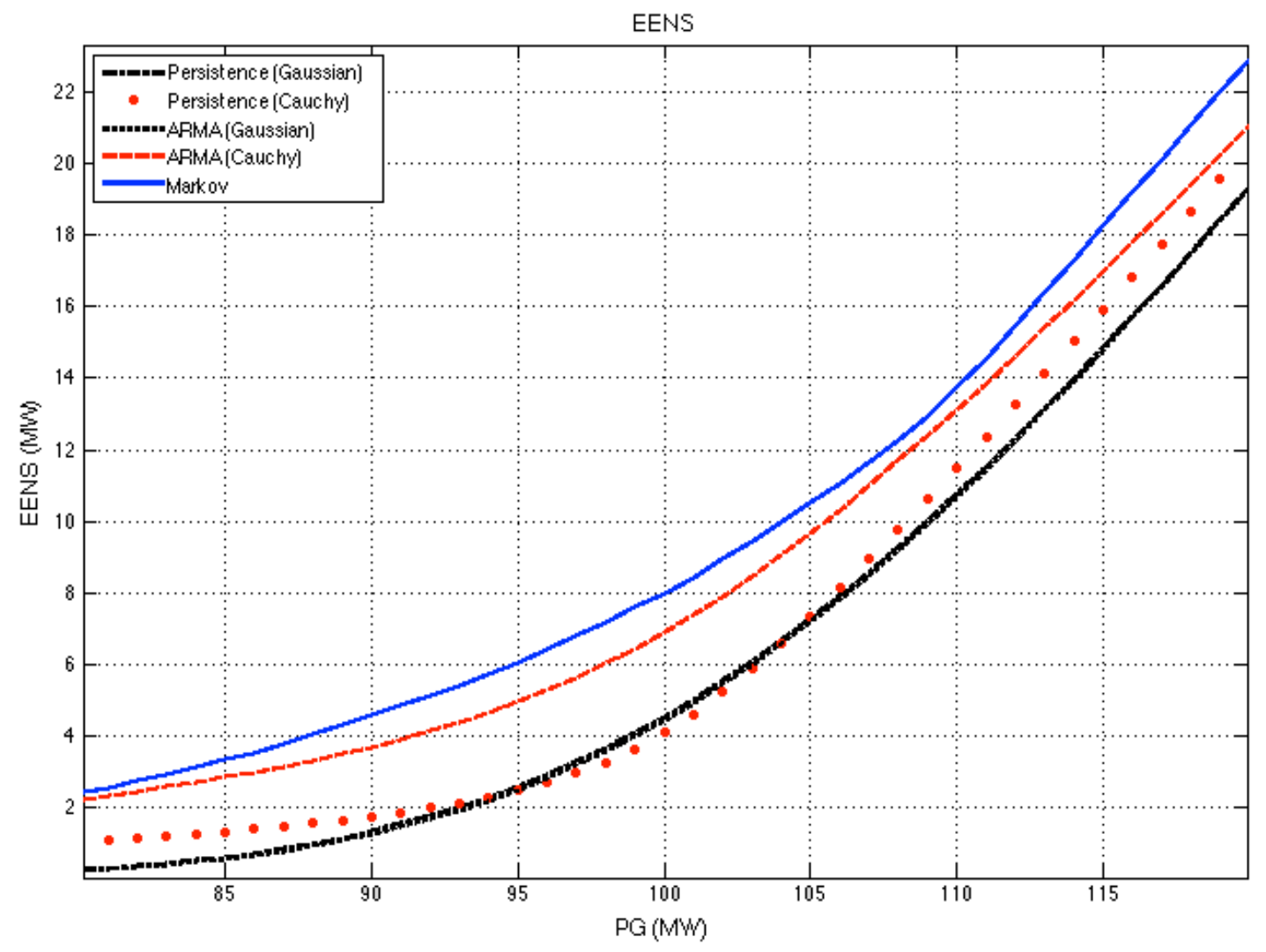

Figure 3.3.2.1: EENS calculated from persistence, Markov Chain, and ARMA models for Wolfe Island wind farm

The plot in figure 14 use a forecasted generation, $\overline{\overline{P G}}$, of $100 \mathrm{MW}$. It compares EENS estimations for the three models with Gaussian and Cauchy distributions used for persistence and ARMA. The results are similar to those in figure 9. For Wolfe Island, the ARMA and persistence EENS is much closer than that of Amaranth. The Markov chain EENS is vertically shifted upward compared to the Amaranth case because a $\overline{\overline{P G}}$ of $93 \mathrm{MW}$ is used for Markov chain as a 
result of the discrete nature of the Markov chain model. In all cases, the EENS is slightly higher than the Amaranth case because of the accuracy of each forecast in the Wolfe Island case is slightly worse.

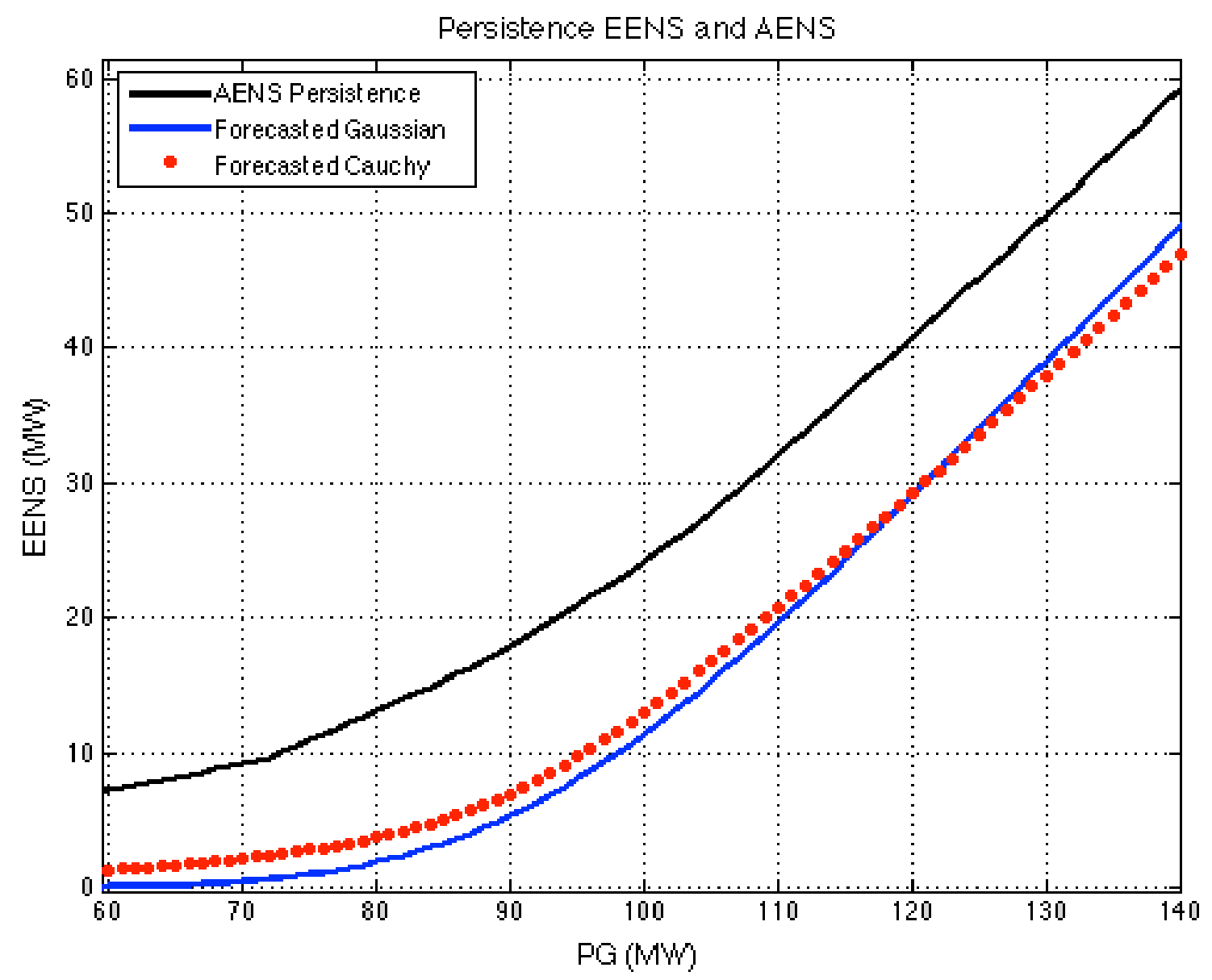

Figure 3.3.2.2: EENS plotted with AENS for persistence model with $\overline{\overline{P G}}$ at $90 M W$ for Wolfe Island wind farm.

Figure 3.3.2.2 shows EENS plotted with AENS for persistence model for $\overline{\overline{P G}}$ at 90MW. AENS is determined in the same way as in figure 3.2.2.2 


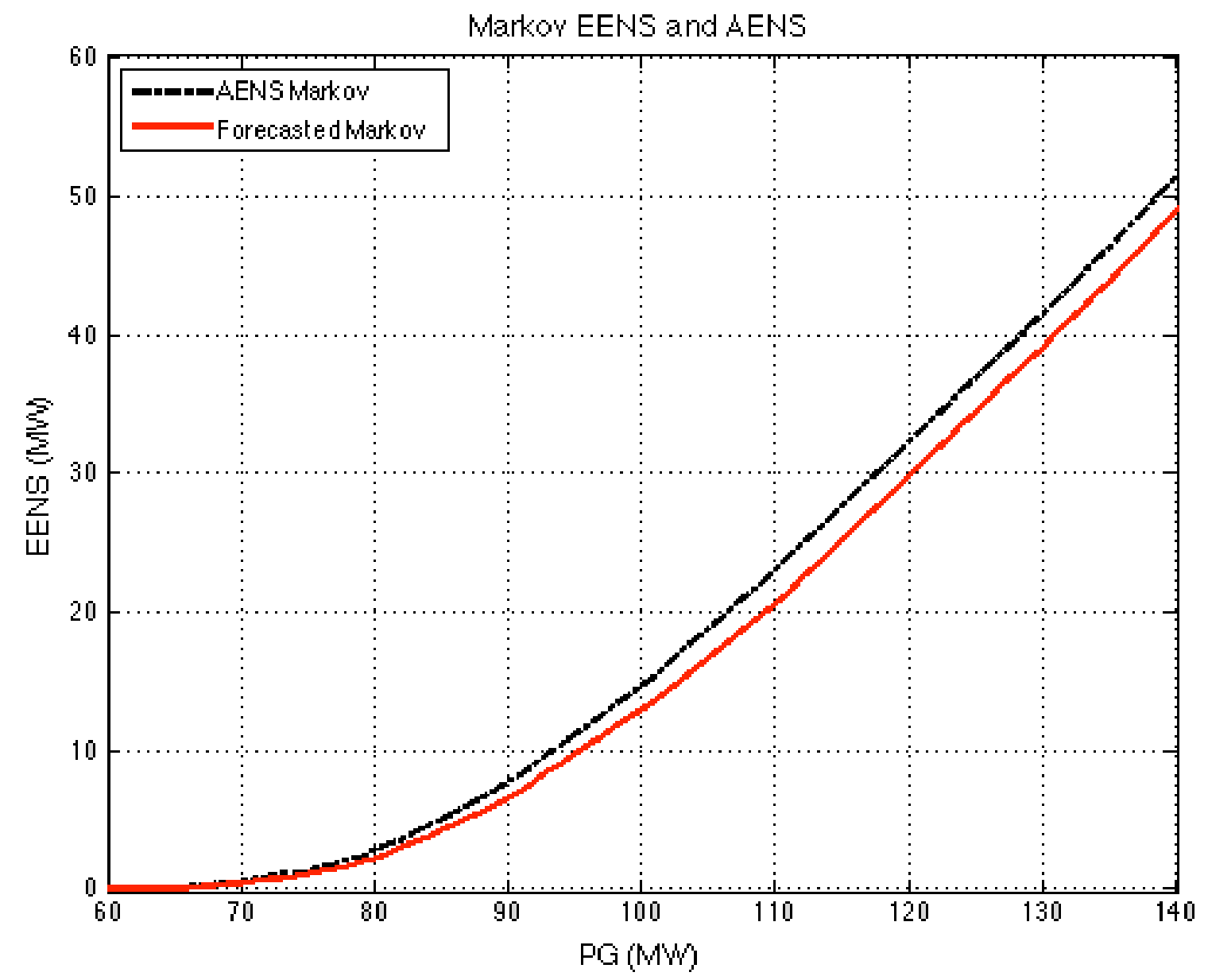

Figure 3.3.2.3: EENS plotted with AENS for Markov chain model with $\overline{\overline{P G}}$ at $90 M W$ for Wolfe Island wind farm

Figure 3.3.2.3 shows EENS plotted with AENS for persistence model for $\overline{\overline{P G}}$ at $90 \mathrm{MW}$. AENS is determined in the same way as in figure 3.2.2.2. A comparison between figure 3.3.2.2 and 3.3.2.3 shows qualitatively that Markov chain model again provides a more accurate EENS estimate than persistence model. 


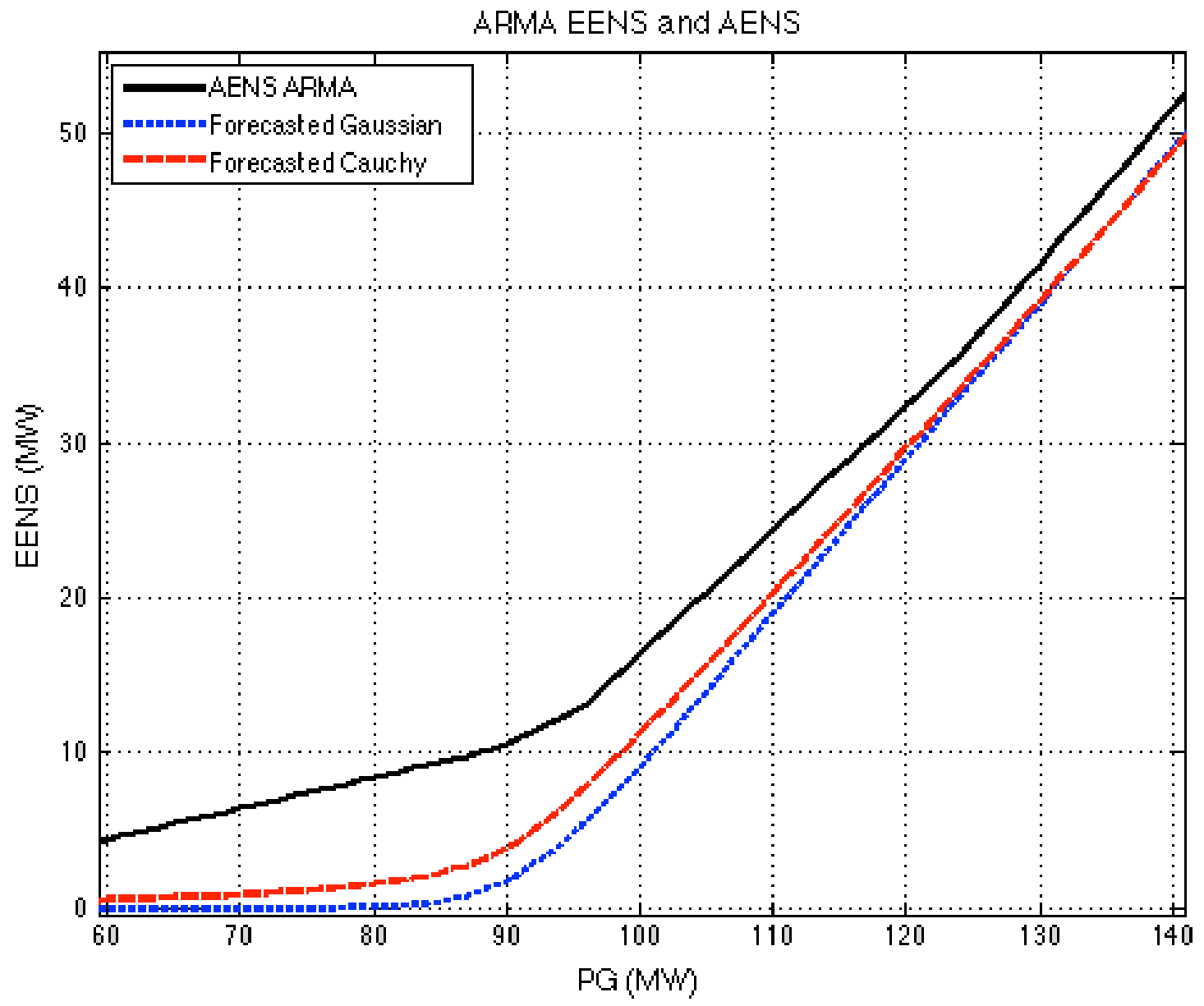

Figure 3.3.2.4: EENS plotted with AENS for ARMA model with $\overline{\overline{P G}}$ at 90MW for Wolfe Island wind farm.

Figure 3.3.2.4 shows EENS plotted with AENS for ARMA model with $\overline{\overline{P G}}$ at $90 \mathrm{MW}$. AENS in figure 3.3.2.4 is determined in the same way as in figure 3.2.2.2. The plot in figure 3.3.2.4 shows that Cauchy distribution provides a better EENS estimate than Gaussian distribution for ARMA model. 
Table 3.3.2.1: NRMSE of EENS vs. AENS for each predictive model and distribution (Amaranth)

\begin{tabular}{|l|l|}
\hline Model & NRMSE (\% of $P G_{\text {nominal) }}$ \\
\hline Persistence (Gaussian) & 6.15 \\
\hline Persistence (Cauchy) & 9.98 \\
\hline Markov Chain & 1.34 \\
\hline ARMA (Gaussian) & 4.58 \\
\hline ARMA (Cauchy) & 4.46 \\
\hline
\end{tabular}

Table 3.3.2.1 gives the NRMSE (as defined in section 3.2.1) between EENS and AENS for each model and distribution with $P G_{\text {sched }}$ ranging between $60 \mathrm{MW}$ and $140 \mathrm{MW}$. It can be seen that the Markov chain model again provides the best estimate of the five scenarios and ARMA also provides a more accurate estimate than persistence model. As with Amaranth wind farm, Cauchy distribution outperforms Gaussian for both persistence and ARMA models.

\subsection{ERCOT System-wide}

\subsubsection{Comparing Forecast Error}

The three models are trained on the hourly training data based on 240 hours of ERCOT's (Electric Reliability Council of Texas) system-wide wind energy output with a nominal power output, $P G_{\text {nominal }}$, of $12212 \mathrm{MW}$.

Each of the three models is used to generate a one-hour-ahead forecast for the duration of the validation data (48 hours). They are plotted against the actual wind generation of the Wolfe Island Wind Farm during that time period. 
In the Markov chain model used, $N$ is chosen to be 100. In the ARMA model used, $p$ and $q$ are chosen to be 2 , and 1 respectively. Plots of each predictive model using the validation data are shown below.

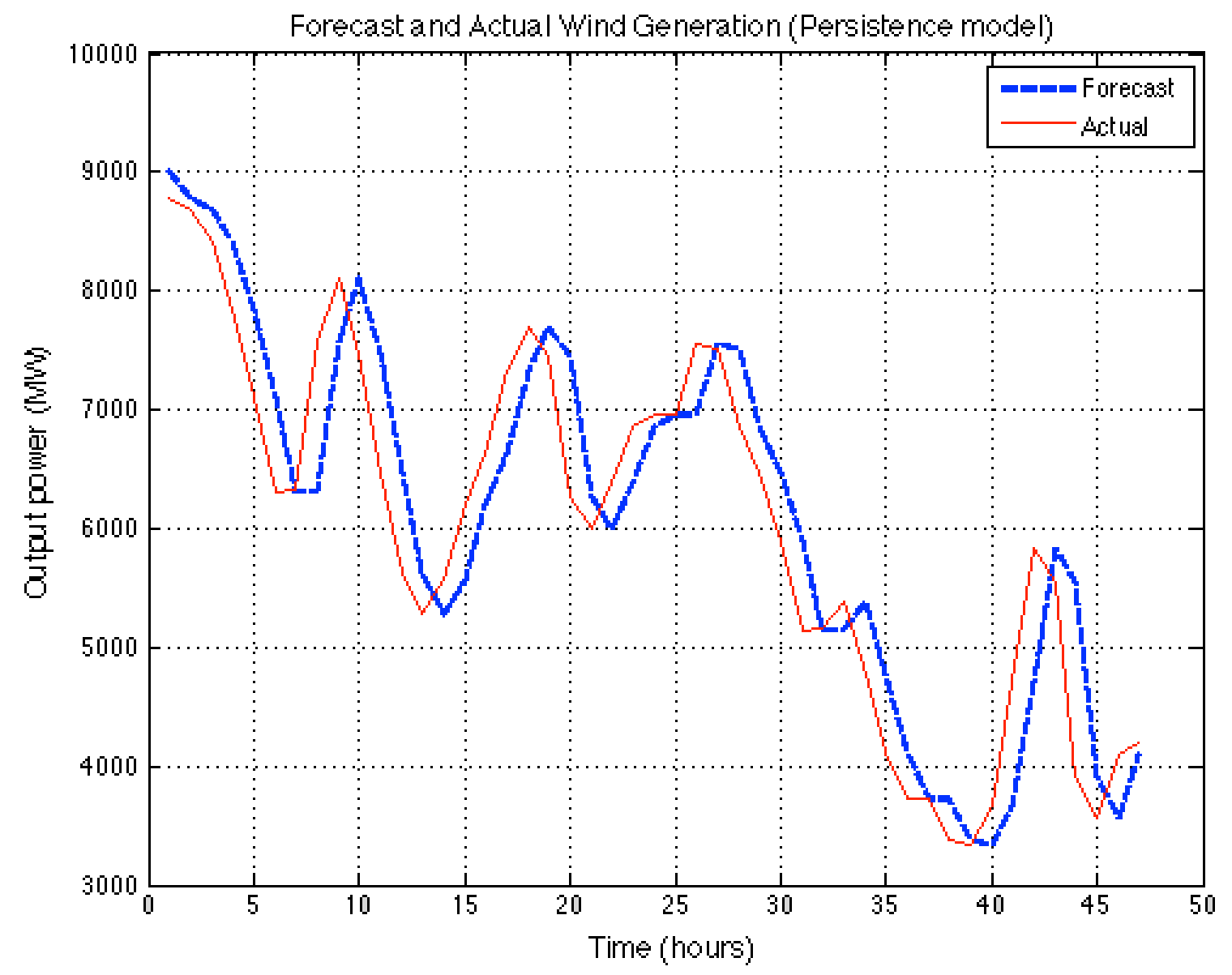

Figure 3.4.1.1: Persistence model wind energy generation forecast compared to actual generation for ERCOT system-wide 


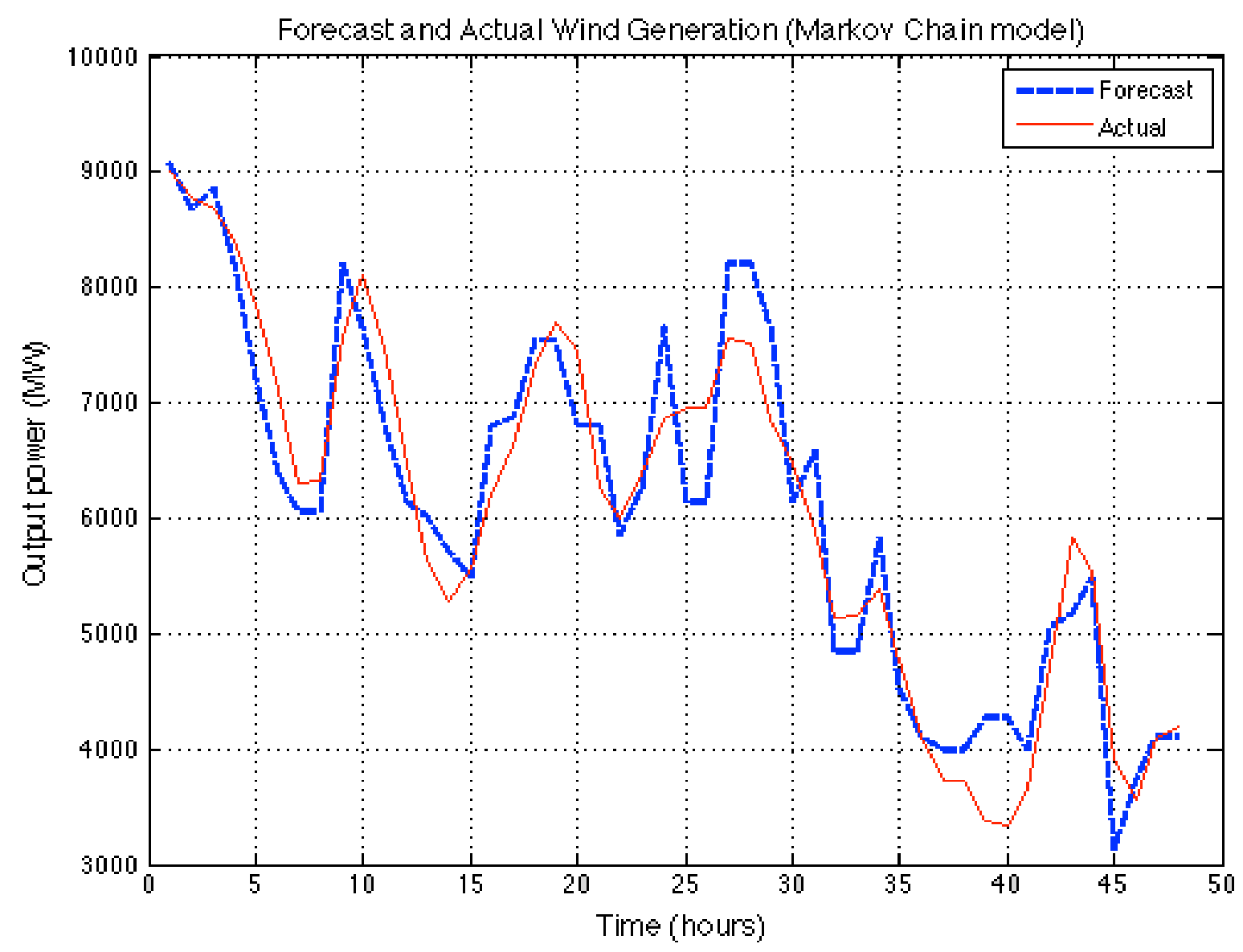

Figure 3.4.1.2: Markov chain model wind energy generation forecast compared to actual generation for ERCOT system-wide 


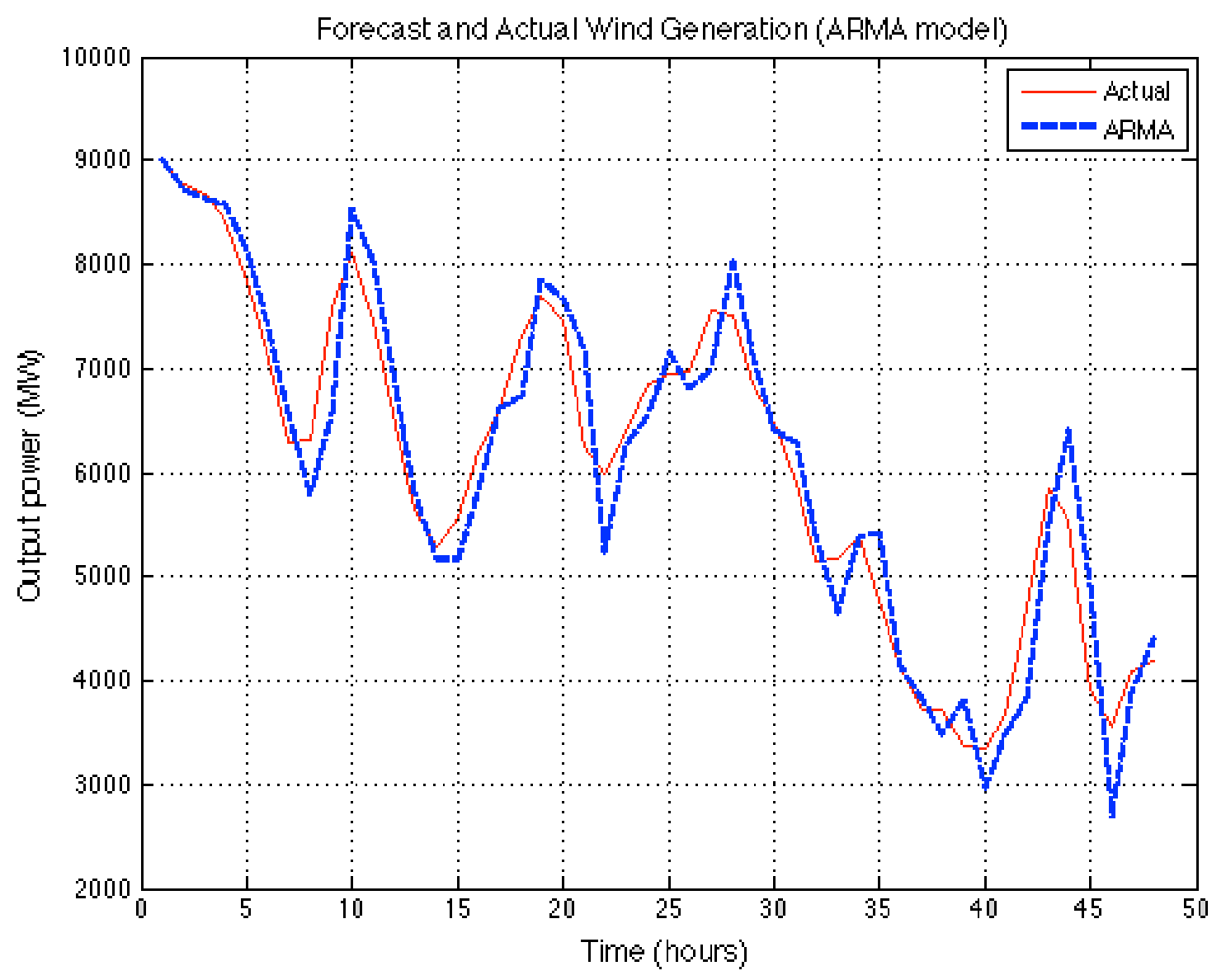

Figure 3.4.1.3: ARMA model wind energy generation forecast compared to actual generation for ERCOT system-wide 
As in table 3.2.1.1, table 3.4.1.1 shows the NRMSE of each model and its predictive accuracy using the data from ERCOT system-wide. The sample size, $m$, is 48 .

Table 3.4.1.1: NRMSE of forecast for each predictive model

\begin{tabular}{|l|l|} 
Model & NRMSE \\
\hline Persistence & 5.0167 \\
\hline Markov & 3.961 \\
\hline ARMA & 3.7641 \\
\hline
\end{tabular}




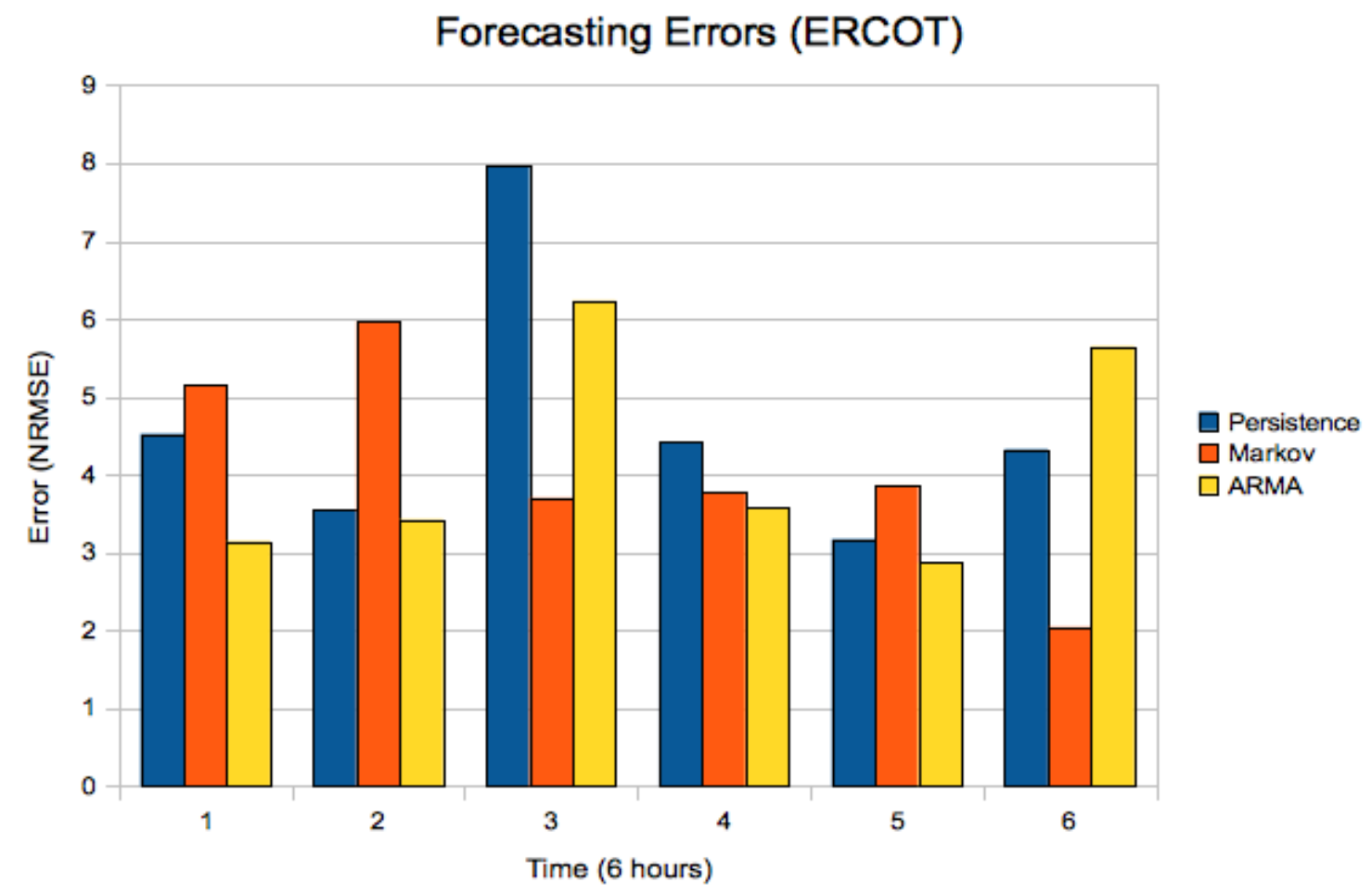

Figure 3.4.1.4: Forecast errors of all three predictive models plotted together for ERCOT system-wide

As in figure 3.2.1.4, figure 3.4.1.4 shows the NRMSE of each predictive model side by side during windows of 6 hours. Unlike figure 3.2.1.4, and figure 3.3.1.4 however, it is not immediately clear which model performs best. Table 3.4.1.1 shows numerically however that Markov chain and ARMA outperform persistence and ARMA performs best overall. 


\subsubsection{Comparing EENS}

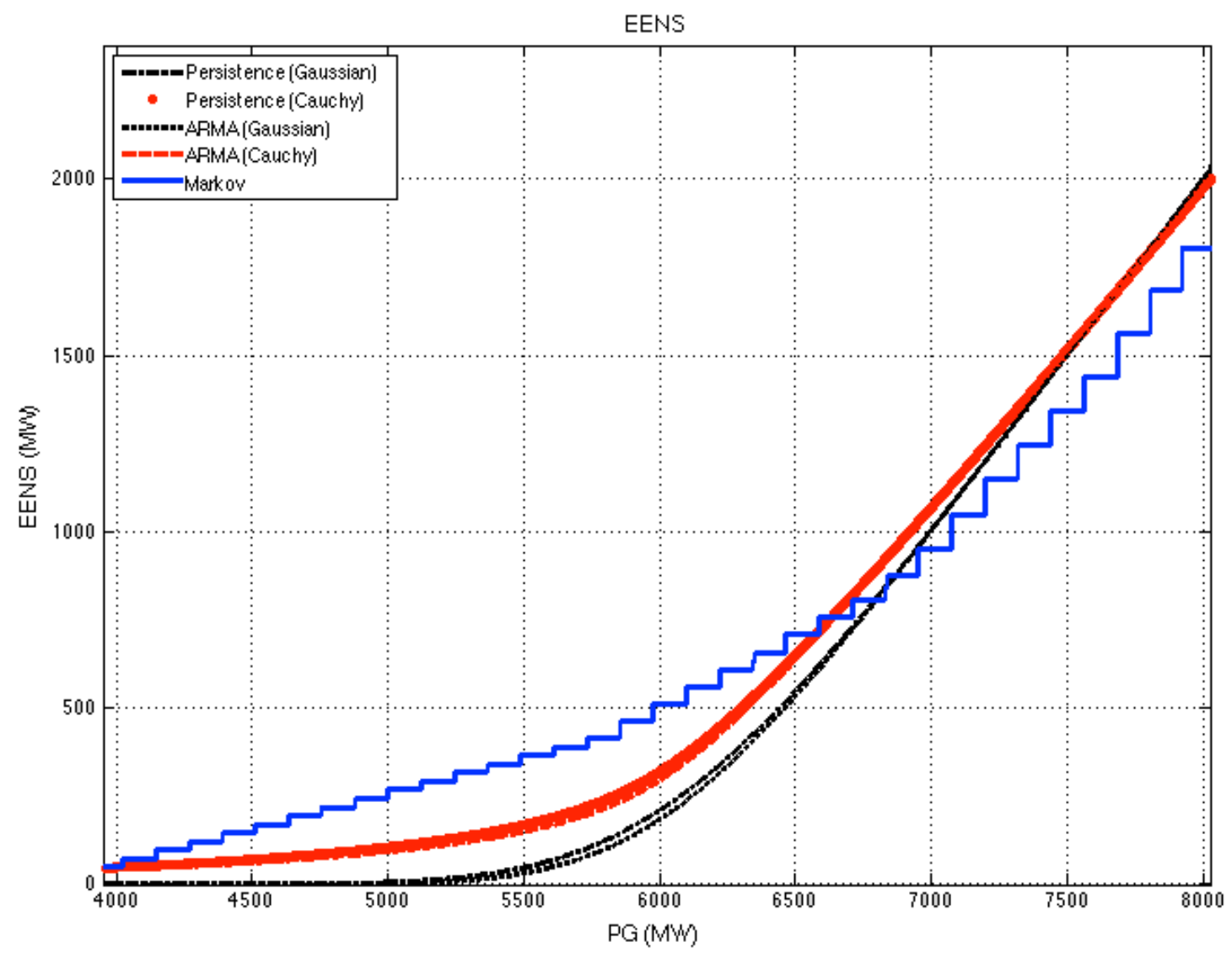

Figure 3.4.2.1: EENS calculated from persistence, Markov Chain, and ARMA models for ERCOT system-wide

The plot in figure 14 use a forecasted generation, $\overline{\overline{P G}}$, of $6000 \mathrm{MW}$. It compares EENS estimations for the three models with Gaussian and Cauchy distributions used for persistence and ARMA. In this scenario, The persistence and ARMA models using Gaussian distributions have lower EENS estimates than with Cauchy distributions for smaller values of $P G$. The Gaussian 
distribution exhibits greater slope in the linear portion starting around $6500 \mathrm{MW}$ and the intersection point is near $7600 \mathrm{MW}$. The Markov chain model exhibits smaller EENS estimates compared to the other two models for values of $P G$ smaller than $6700 \mathrm{MW}$, and higher EENS estimates for higher values of $P G$. It should be noted that the Markov chain plot exhibits a staircase pattern because the number of bins, $N$, is small relative to the nominal output power.

No comparisons between AENS and EENS are generated for the ERCOT system-wide case study because the training data is not sufficiently large for accurate results and conclusions to be drawn from it.

\subsubsection{Comparison with Previous Work}

The previously referenced paper [11] studied the use of ARMA models on Texas' ERCOT wind energy generators. In that paper, accuracy of the model was defined as "the number of forecasts made by the model that carry an error less than a preset value, expressed as a percentage of the total number of forecasts made. For example, if 100 hour-ahead forecasts are made with the number of counts of the error less than $25 \%$ being equal to 90 ; the accuracy of the forecast is said to $90 \%$ in the $25 \%$ error limit. [11]" This definition was used for persistence, Markov chain, and ARMA models to evaluate their performance relative to the results shown in [11].

Table 3.4.3.1: Accuracy with 25\% error limit for one hour ahead forecast in [11].

\begin{tabular}{|l|l|l|l|}
\hline January & April & July & October \\
\hline 83.888 & 82.491 & 73.032 & 82.253 \\
\hline
\end{tabular}


Table 3.4.3.2: Accuracy with 25\% error limit for one hour ahead forecast in for persistence, Markov chain, and ARMA models.

\begin{tabular}{|l|l|}
\hline Model & Accuracy \\
\hline Persistence & 99.792 \\
\hline Markov chain & 95.833 \\
\hline ARMA & 99.792 \\
\hline
\end{tabular}

The results in table 3.4.3.1 and table 3.4.3.2 show that the three models examined here provided good estimates of WEG generation for the ERCOT system. There are however, a few caveats about the results shown in table 3.4.3.2. The size of the training data and the validation data used is significantly smaller than was used in [11]. Also, the data used for table 3.4.3.1 is from 2006 while the data used for table 3.4.3.2 is from 2014. The ERCOT grid has changed significantly since then and the total WEG capacity has increased since then. 


\section{Conclusions}

The ability to accurately forecast wind energy generator power generation as well as the expected energy not served of a wind energy generator is very important in quantifying the risks associated with wind energy. Expected energy not served calculation requires an error distribution for the forecasted wind energy generator power generation. To this end, various predictive models can be used to produce this error distribution. Although much work has been done on evaluating and comparing predictive models for wind energy generator power forecasting, their effects on expected energy not served has been thus far unexplored.

In this thesis, Markov chain model, and ARMA models are used to produce a point forecast for the one-hour-ahead horizon as well as an error distribution that could be used for expected energy not served calculation considering a wind farm data from Ontario, Canada. These two models were compared to persistence model which was used to establish a benchmark.

Of the two predictive models examined, Markov chain model provided the better forecast, defined here as least normalized root-mean-square error. Both Markov chain and ARMA models outperformed persistence model and produce an accurate forecast. As evidenced by tables 3.2.2.1, and 3.3.2.1, Markov chain also provides the best estimation of expected energy not served as compared to actual energy not served, again as defined as least normalized root-mean-square error. Again, Markov chain and ARMA models both outperform persistence model. Markov chain model in particular gives a very accurate error distribution which is useful in producing accurate expected energy not served calculations. 
Two other case studies were examined. The first involved data from Ontario's Wolfe Island wind farm. The results from that were similar to those of the Amaranth wind farm though slightly less accurate. As a result, the estimated expected energy not served skewed higher. The second involved data from Texas' ERCOT system. These results were also compared to the results from [11] which used older data from the ERCOT system. In most cases, Markov chain model is recommended in order to produce the best expected energy not served estimate based on the case studies examined. It should be noted however, that the most suitable model can differ depending on the specific application or location. This thesis provides a methodology by which the best model for estimating expected energy not served can be determined for any given situation or models.

A closed-form solution was found for expected energy not served when using a Cauchy distribution. A solution for expected energy not served was also found when using a Gaussian distribution. It is not fully closed-form because of the presence of the error function within the solution but look-up tables can be used for efficient calculation. Both solutions aid in reducing the computational complexity of calculating expected energy not served which is important in solving for OPF. 


\subsection{Simulation Data}

\section{APPENDIX}

\subsubsection{Amaranth Wind Farm Raw Data}

\subsubsection{Training Data}

The data below is the hourly training data from Amaranth wind farm used to create the Markov chain and ARMA models. It is used to generate the plots and tables section 3.2.

\begin{tabular}{|c|c|c|c|c|c|c|c|c|c|c|c|c|c|c|c|c|c|c|c|c|c|c|c|}
\hline \multicolumn{24}{|c|}{$\underline{\text { Hour }}$} \\
\hline 1 & 2 & 3 & 4 & 5 & 6 & 7 & 8 & 9 & 10 & 11 & 12 & 13 & 14 & 15 & 16 & 17 & 18 & 19 & 20 & 21 & 22 & 23 & 24 \\
\hline 16 & 23 & 12 & 10 & 8 & 8 & 9 & 12 & 12 & 8 & 6 & 1 & 0 & 0 & 2 & 4 & 13 & 10 & 5 & 2 & 11 & 30 & 33 & 61 \\
\hline 67 & 41 & 40 & 30 & 22 & 28 & 27 & 24 & 7 & 17 & 33 & 17 & 4 & 1 & 1 & 0 & 0 & 2 & 2 & 3 & 9 & 4 & 5 & 19 \\
\hline 34 & 54 & 78 & 118 & 124 & 135 & 155 & 155 & 165 & 169 & 175 & 181 & 183 & 182 & 181 & 182 & 182 & 179 & 181 & 168 & 146 & 128 & 93 & 79 \\
\hline 64 & 75 & 116 & 111 & 91 & 61 & 59 & 80 & 119 & 108 & 117 & 125 & 133 & 107 & 95 & 115 & 104 & 114 & 117 & 129 & 155 & 158 & 144 & 132 \\
\hline 131 & 130 & 120 & 111 & 116 & 123 & 109 & 119 & 128 & 135 & 145 & 131 & 106 & 115 & 115 & 122 & 104 & 85 & 74 & 64 & 58 & 82 & 83 & 83 \\
\hline 75 & 72 & 83 & 78 & 68 & 70 & 69 & 82 & 82 & 79 & 74 & 50 & 58 & 56 & 40 & 58 & 72 & 100 & 97 & 71 & 83 & 51 & 36 & 32 \\
\hline 40 & 50 & 56 & 59 & 72 & 77 & 66 & 57 & 85 & 124 & 118 & 116 & 119 & 125 & 133 & 132 & 136 & 131 & 114 & 74 & 76 & 92 & 79 & 90 \\
\hline 56 & 40 & 36 & 19 & 12 & 21 & 22 & 20 & 36 & 47 & 63 & 52 & 50 & 62 & 82 & 95 & 92 & 61 & 31 & 29 & 33 & 25 & 27 & 21 \\
\hline 19 & 14 & 13 & 8 & 1 & 8 & 26 & 19 & 1 & 2 & 3 & 9 & 12 & 16 & 21 & 33 & 37 & 31 & 23 & 25 & 24 & 40 & 57 & 77 \\
\hline 73 & 84 & 72 & 80 & 94 & 110 & 87 & 73 & 87 & 74 & 71 & 89 & 135 & 128 & 111 & 79 & 42 & 27 & 17 & 21 & 24 & 17 & 17 & 18 \\
\hline 30 & 37 & 39 & 45 & 39 & 37 & 37 & 35 & 43 & 38 & 33 & 38 & 38 & 29 & 35 & 62 & 68 & 63 & 58 & 47 & 47 & 38 & 32 & 26 \\
\hline 33 & 49 & 53 & 56 & 46 & 38 & 33 & 31 & 25 & 15 & 26 & 63 & 110 & 117 & 98 & 80 & 67 & 48 & 34 & 28 & 32 & 29 & 32 & 21 \\
\hline 12 & 6 & 4 & 4 & 7 & 8 & 10 & 13 & 9 & 6 & 8 & 8 & 7 & 2 & 10 & 21 & 27 & 35 & 27 & 23 & 21 & 16 & 14 & 17 \\
\hline 22 & 28 & 47 & 49 & 54 & 50 & 45 & 22 & 17 & 20 & 20 & 22 & 17 & 14 & 15 & 11 & 9 & 7 & 9 & 10 & 23 & 16 & 20 & 24 \\
\hline 18 & 20 & 31 & 23 & 19 & 15 & 11 & 15 & 5 & 0 & 1 & 3 & 10 & 17 & 22 & 36 & 35 & 31 & 36 & 63 & 71 & 94 & 101 & 87 \\
\hline 85 & 92 & 104 & 96 & 91 & 81 & 82 & 93 & 89 & 75 & 82 & 51 & 74 & 101 & 94 & 89 & 69 & 48 & 46 & 52 & 59 & 69 & 62 & 72 \\
\hline 72 & 100 & 121 & 146 & 164 & 144 & 170 & 169 & 183 & 182 & 180 & 182 & 182 & 182 & 175 & 144 & 124 & 146 & 113 & 105 & 99 & 119 & 123 & 120 \\
\hline 124 & 101 & 54 & 54 & 50 & 38 & 46 & 33 & 34 & 47 & 53 & 97 & 139 & 163 & 176 & 177 & 176 & 156 & 124 & 90 & 85 & 113 & 108 & 95 \\
\hline 90 & 71 & 54 & 65 & 84 & 46 & 24 & 24 & 48 & 121 & 121 & 98 & 108 & 138 & 150 & 153 & 150 & 141 & 81 & 56 & 33 & 49 & 67 & 69 \\
\hline 62 & 63 & 127 & 131 & 133 & 104 & 146 & 160 & 155 & 139 & 144 & 135 & 114 & 112 & 126 & 123 & 121 & 119 & 99 & 69 & 67 & 68 & 78 & 35 \\
\hline
\end{tabular}




\begin{tabular}{|c|c|c|c|c|c|c|c|c|c|c|c|c|c|c|c|c|c|c|c|c|c|c|c|}
\hline 44 & 47 & 48 & 56 & 9 & 48 & 46 & 67 & 46 & 96 & 79 & 79 & 120 & 114 & 96 & 89 & 84 & 76 & 67 & 56 & 40 & 24 & 18 & 13 \\
\hline 13 & 11 & 16 & 13 & 3 & 0 & 1 & 2 & 10 & 23 & 34 & 45 & 50 & 63 & 68 & 73 & 61 & 80 & 75 & 57 & 128 & 168 & 171 & 143 \\
\hline 6 & 38 & 42 & 64 & 56 & 94 & 90 & 91 & 90 & 39 & 87 & 91 & 92 & 91 & ( & 87 & 0 & 86 & 12 & 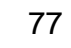 & 34 & - & 9 & \\
\hline 1 & 0 & 0 & 0 & 0 & 2 & 4 & 14 & 25 & 29 & 37 & 52 & 79 & 103 & 55 & 46 & 116 & 148 & 13 & 1 & 54 & 43 & 64 & 102 \\
\hline 97 & 101 & 86 & 54 & 54 & 14 & 7 & 1 & 0 & 0 & 1 & 2 & 4 & 2 & 3 & 3 & 2 & 3 & 28 & 32 & 39 & 17 & 38 & 29 \\
\hline 17 & 36 & 25 & 24 & 29 & 37 & 27 & 55 & 22 & 83 & 70 & 50 & 33 & 35 & 51 & 88 & 15 & 16 & 23 & 34 & 26 & 83 & 93 & 73 \\
\hline 3 & 54 & 88 & 12 & 96 & 29 & 98 & 110 & 108 & 13 & 89 & 98 & 100 & 146 & 45 & 157 & 50 & 153 & 143 & 162 & 152 & 146 & 97 & 38 \\
\hline 10 & 5 & 3 & 2 & 1 & 1 & 0 & 0 & 0 & 0 & 0 & 0 & 0 & 0 & 0 & 0 & 0 & 0 & 0 & 0 & 0 & 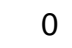 & 0 & O \\
\hline 0 & 0 & 0 & 0 & 0 & 0 & 1 & 54 & 51 & 29 & 36 & 06 & 143 & 127 & 87 & 79 & 04 & 95 & 91 & 79 & 86 & 104 & 103 & 111 \\
\hline 97 & 89 & 57 & 57 & 5 & 40 & 27 & 39 & 56 & 52 & 26 & 18 & 28 & 17 & 15 & 4 & 0 & 2 & 4 & 15 & 42 & 52 & 60 & 68 \\
\hline 76 & 96 & 19 & 45 & 41 & 130 & 17 & 92 & 62 & 45 & 31 & 43 & 38 & 56 & 86 & 82 & 31 & 38 & 50 & 81 & 109 & 135 & 161 & 162 \\
\hline 1 & 61 & 50 & 169 & 0 & 169 & 67 & 134 & 80 & 45 & 55 & 51 & 59 & 109 & 111 & 122 & 118 & 98 & 60 & 34 & 31 & 44 & 37 & 22 \\
\hline 37 & 31 & 20 & 17 & 20 & 16 & 19 & 16 & 32 & 29 & 12 & 5 & 5 & 9 & 14 & 28 & 53 & 44 & 49 & 38 & 47 & 33 & 147 & 135 \\
\hline 2 & 160 & 66 & 158 & 40 & 135 & 37 & 181 & 166 & 21 & 156 & 71 & 176 & 182 & 180 & 182 & 175 & 80 & 167 & 20 & 26 & 124 & 117 & 138 \\
\hline 8 & 87 & 74 & 63 & 84 & 134 & 32 & 184 & 184 & 84 & 180 & 67 & 177 & 184 & 182 & 183 & 184 & 184 & 175 & 75 & 175 & 174 & 174 & 162 \\
\hline 6 & 138 & 26 & 142 & 17 & 125 & 42 & 155 & 156 & 62 & 163 & 62 & 164 & 164 & 159 & 135 & 131 & 116 & 93 & 84 & 55 & 25 & 18 & 20 \\
\hline 20 & 16 & 7 & 2 & 1 & 0 & 0 & 0 & 0 & 0 & 0 & 0 & 1 & 1 & 0 & 0 & 1 & 6 & 41 & 69 & 62 & 59 & 62 & 76 \\
\hline 68 & 67 & 74 & 88 & 86 & 78 & 50 & 35 & 19 & 13 & 20 & 34 & 43 & 41 & 47 & 51 & 72 & 66 & 59 & 59 & 82 & 101 & 115 & 107 \\
\hline 82 & 104 & 29 & 130 & 24 & 109 & 13 & 78 & 62 & 65 & 99 & 122 & 113 & 100 & 96 & 110 & 123 & 101 & 89 & 81 & 86 & 114 & 115 & 68 \\
\hline 86 & 93 & 60 & 95 & 03 & 111 & 80 & 23 & 10 & 11 & 18 & 19 & 23 & 51 & 97 & 108 & 126 & 122 & 122 & 14 & 119 & 126 & 143 & 105 \\
\hline 5 & 47 & 52 & 38 & 0 & 14 & 19 & 22 & 26 & 30 & 56 & 27 & 156 & 180 & 79 & 13 & & 3 & & 38 & & 45 & 6 & 35 \\
\hline 62 & 71 & 84 & 67 & 45 & 32 & 25 & 5 & 2 & 6 & 7 & 3 & 11 & 14 & 24 & 30 & 38 & 39 & 40 & 54 & 56 & 47 & 50 & 63 \\
\hline 86 & 61 & 1 & 69 & 44 & 21 & 35 & 53 & 40 & 28 & 20 & 21 & 31 & 29 & 28 & 35 & 34 & 35 & 26 & 17 & 20 & 31 & 35 & 57 \\
\hline 4 & 126 & 21 & 09 & 8 & 51 & 58 & 35 & 31 & 34 & 31 & 57 & 109 & 146 & 153 & 153 & 143 & 133 & 126 & 108 & 103 & 104 & 88 & 75 \\
\hline 64 & 50 & 6 & 53 & 2 & 48 & 42 & 43 & 38 & 40 & 32 & 26 & 23 & 19 & 21 & 21 & 21 & 18 & 14 & 17 & 20 & 31 & 24 & 28 \\
\hline 20 & 15 & 5 & 22 & 3 & 26 & 21 & 22 & 20 & 28 & 43 & 66 & 72 & 86 & 139 & 167 & 152 & 125 & 80 & 57 & 72 & 70 & 92 & 124 \\
\hline 25 & 120 & 114 & 121 & 18 & 101 & 68 & 74 & 60 & 36 & 31 & 35 & 35 & 25 & 21 & 18 & 1 & 17 & 19 & 34 & $4 r$ & 65 & 71 & 90 \\
\hline 71 & 46 & 29 & 20 & 50 & 64 & 54 & 37 & 40 & 16 & 14 & 24 & 58 & 77 & 68 & 60 & 47 & 36 & 33 & 47 & 58 & 90 & 107 & 112 \\
\hline 9 & 72 & 71 & 57 & 54 & 43 & 56 & 51 & 52 & 62 & 57 & 64 & 63 & 75 & 82 & 87 & 89 & 52 & 36 & 48 & 66 & 78 & 80 & 89 \\
\hline 8 & 65 & 55 & 71 & 70 & 71 & 53 & 39 & 24 & 7 & 19 & 52 & 78 & 87 & 73 & 59 & 44 & 30 & 19 & 12 & 18 & 32 & 52 & 59 \\
\hline 56 & 53 & 0 & 28 & 32 & 42 & 39 & 16 & 10 & 10 & 2 & 4 & 3 & 8 & 10 & 10 & 6 & 24 & 28 & 17 & 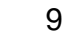 & 37 & 38 & 32 \\
\hline 24 & 27 & 34 & 25 & 17 & 24 & 22 & 4 & 0 & 2 & 3 & 10 & 16 & 16 & 12 & 7 & 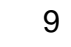 & 19 & 24 & 19 & 3 & $\perp$ & 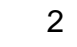 & 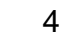 \\
\hline 12 & 16 & 14 & 9 & 9 & 2 & 2 & 1 & 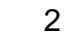 & 4 & 12 & 9 & 5 & 6 & & 9 & 7 & & 9 & & 7 & 11 & 12 & 29 \\
\hline 21 & 26 & 33 & 34 & 23 & 24 & 20 & 9 & 1 & 2 & 5 & 11 & 12 & 9 & 14 & 16 & 22 & 15 & 9 & 4 & 3 & 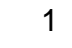 & 1 & 1 \\
\hline 0 & 0 & 3 & 0 & 8 & 4 & 7 & 3 & a & 1 & 3 & 0 & 10 & 22 & 0 & 11 & 37 & 22 & 16 & 15 & 15 & 26 & 32 & 45 \\
\hline
\end{tabular}


$\begin{array}{llllllllllllllllllllllllllll}37 & 49 & 58 & 69 & 66 & 50 & 31 & 43 & 63 & 105 & 151 & 105 & 54 & 28 & 11 & 2 & 4 & 3 & 3 & 5 & 5 & 5 & 14 & 22\end{array}$

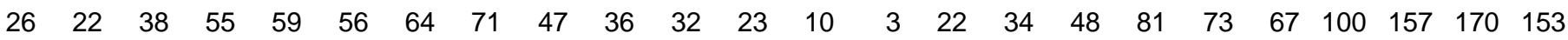

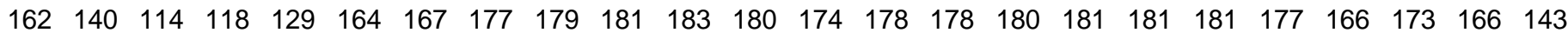
$\begin{array}{lllllllllllllllllllllllll}101 & 80 & 70 & 76 & 93 & 90 & 72 & 68 & 85 & 126 & 128 & 118 & 137 & 149 & 150 & 150 & 155 & 135 & 102 & 59 & 37 & 29 & 23 & 10\end{array}$

$\begin{array}{llllllllllllllllllllllll}2 & 3 & 15 & 31 & 36 & 34 & 38 & 35 & 56 & 47 & 58 & 71 & 72 & 76 & 64 & 53 & 53 & 51 & 59 & 65 & 72 & 82 & 97 & 109\end{array}$ $\begin{array}{llllllllllllllllllllllll}104 & 105 & 107 & 103 & 148 & 155 & 155 & 123 & 114 & 134 & 169 & 181 & 181 & 182 & 183 & 186 & 177 & 161 & 131 & 86 & 75 & 45 & 38 & 47\end{array}$ $\begin{array}{llllllllllllllllllllllll}58 & 49 & 72 & 79 & 61 & 38 & 28 & 16 & 32 & 79 & 115 & 122 & 126 & 117 & 109 & 81 & 40 & 33 & 30 & 20 & 21 & 33 & 37 & 25\end{array}$ $\begin{array}{lllllllllllllllllllllllllll}13 & 8 & 17 & 31 & 40 & 33 & 17 & 11 & 14 & 11 & 4 & 0 & 0 & 0 & 0 & 0 & 2 & 4 & 10 & 7 & 9 & 5 & 5 & 8\end{array}$ $\begin{array}{lllllllllllllllllllllllll}14 & 22 & 46 & 52 & 37 & 29 & 21 & 11 & 16 & 32 & 46 & 40 & 35 & 33 & 22 & 20 & 20 & 17 & 18 & 20 & 28 & 35 & 34 & 34\end{array}$ $\begin{array}{llllllllllllllllllllllllll}46 & 50 & 44 & 40 & 34 & 34 & 37 & 21 & 20 & 22 & 18 & 30 & 35 & 26 & 25 & 28 & 21 & 21 & 21 & 22 & 33 & 34 & 33 & 46\end{array}$ $\begin{array}{llllllllllllllllllllllll}55 & 61 & 65 & 63 & 54 & 35 & 16 & 4 & 2 & 2 & 2 & 7 & 18 & 32 & 48 & 62 & 82 & 83 & 51 & 48 & 117 & 72 & 55 & 34\end{array}$ $\begin{array}{llllllllllllllllllllllllllll}33 & 33 & 42 & 67 & 62 & 46 & 25 & 8 & 9 & 8 & 35 & 65 & 95 & 46 & 1 & 4 & 52 & 68 & 23 & 22 & 19 & 13 & 13 & 26\end{array}$ $\begin{array}{llllllllllllllllllllllllll}25 & 15 & 19 & 25 & 23 & 19 & 13 & 7 & 8 & 6 & 17 & 26 & 44 & 51 & 62 & 69 & 137 & 129 & 85 & 43 & 54 & 35 & 23 & 45\end{array}$ $\begin{array}{llllllllllllllllllllllll}42 & 31 & 26 & 37 & 54 & 43 & 55 & 65 & 101 & 81 & 33 & 58 & 74 & 102 & 125 & 141 & 135 & 143 & 145 & 151 & 147 & 138 & 128 & 111\end{array}$ $\begin{array}{llllllllllllllllllllllllll}111 & 136 & 108 & 95 & 121 & 93 & 106 & 116 & 131 & 133 & 117 & 97 & 96 & 89 & 87 & 83 & 79 & 75 & 63 & 48 & 33 & 31 & 35 & 44\end{array}$ $\begin{array}{llllllllllllllllllllllllll}49 & 42 & 32 & 32 & 42 & 38 & 23 & 25 & 62 & 62 & 71 & 69 & 77 & 79 & 67 & 60 & 70 & 72 & 66 & 45 & 44 & 41 & 40 & 42\end{array}$ $\begin{array}{llllllllllllllllllllllllll}53 & 53 & 41 & 42 & 50 & 35 & 17 & 19 & 57 & 60 & 59 & 61 & 72 & 75 & 72 & 63 & 49 & 31 & 19 & 14 & 19 & 24 & 30 & 39\end{array}$ $\begin{array}{llllllllllllllllllllllllllll}41 & 41 & 43 & 26 & 17 & 13 & 10 & 3 & 0 & 0 & 0 & 0 & 0 & 0 & 0 & 1 & 1 & 3 & 3 & 3 & 3 & 2 & 6 & 20\end{array}$ $\begin{array}{lllllllllllllllllllllllll}50 & 56 & 52 & 50 & 45 & 55 & 44 & 31 & 43 & 57 & 46 & 39 & 39 & 32 & 24 & 12 & 8 & 51 & 16 & 27 & 19 & 34 & 40 & 34\end{array}$ $\begin{array}{llllllllllllllllllllllll}21 & 64 & 94 & 75 & 67 & 67 & 79 & 89 & 136 & 129 & 103 & 106 & 82 & 65 & 84 & 119 & 109 & 74 & 61 & 44 & 32 & 51 & 63 & 64\end{array}$ $\begin{array}{llllllllllllllllllllllll}54 & 40 & 45 & 50 & 46 & 30 & 27 & 38 & 28 & 27 & 38 & 46 & 56 & 63 & 64 & 81 & 100 & 100 & 68 & 39 & 60 & 80 & 90 & 104\end{array}$ $\begin{array}{llllllllllllllllllllllll}96 & 95 & 103 & 105 & 78 & 58 & 40 & 29 & 27 & 44 & 90 & 98 & 95 & 108 & 149 & 160 & 111 & 75 & 80 & 93 & 59 & 65 & 82 & 40\end{array}$ $\begin{array}{lllllllllllllllllllllllll}64 & 114 & 75 & 36 & 33 & 31 & 56 & 57 & 35 & 39 & 62 & 96 & 91 & 64 & 49 & 63 & 78 & 46 & 26 & 34 & 25 & 27 & 36 & 32\end{array}$

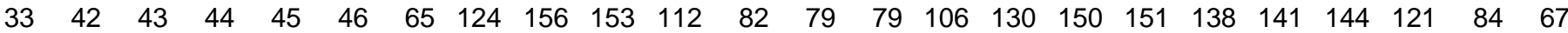
$\begin{array}{llllllllllllllllllllllll}61 & 45 & 36 & 35 & 42 & 62 & 57 & 53 & 58 & 95 & 98 & 108 & 109 & 108 & 113 & 130 & 130 & 122 & 104 & 81 & 43 & 34 & 49 & 67\end{array}$ $\begin{array}{llllllllllllllllllllllll}63 & 52 & 34 & 34 & 26 & 20 & 11 & 15 & 28 & 30 & 26 & 22 & 23 & 19 & 21 & 17 & 14 & 19 & 15 & 12 & 5 & 7 & 5 & 9\end{array}$ $\begin{array}{llllllllllllllllllllllllll}18 & 24 & 23 & 37 & 43 & 37 & 34 & 21 & 12 & 7 & 10 & 18 & 18 & 13 & 17 & 19 & 20 & 20 & 32 & 49 & 62 & 62 & 64 & 72\end{array}$ $\begin{array}{llllllllllllllllllllllll}55 & 65 & 67 & 73 & 80 & 94 & 77 & 78 & 113 & 135 & 146 & 155 & 150 & 157 & 146 & 120 & 104 & 117 & 124 & 111 & 68 & 49 & 71 & 53\end{array}$ $\begin{array}{lllllllllllllllllllllllll}30 & 30 & 21 & 8 & 8 & 6 & 11 & 11 & 13 & 13 & 12 & 8 & 11 & 10 & 15 & 10 & 10 & 11 & 11 & 15 & 20 & 12 & 6 & 3\end{array}$ $\begin{array}{llllllllllllllllllllllllllll}2 & 2 & 5 & 9 & 12 & 13 & 9 & 6 & 10 & 6 & 4 & 6 & 7 & 11 & 6 & 5 & 8 & 3 & 2 & 1 & 3 & 4 & 10 & 9\end{array}$ $\begin{array}{llllllllllllllllllllllllll}8 & 12 & 16 & 14 & 16 & 12 & 3 & 3 & 4 & 2 & 0 & 0 & 0 & 0 & 1 & 2 & 4 & 22 & 38 & 30 & 30 & 35 & 42 & 48\end{array}$ $\begin{array}{llllllllllllllllllllllll}48 & 73 & 88 & 83 & 101 & 96 & 56 & 34 & 16 & 32 & 23 & 40 & 36 & 30 & 33 & 43 & 59 & 80 & 60 & 71 & 49 & 35 & 19 & 11\end{array}$ $\begin{array}{rrrrrrrrrrrrrrrrrrrrrrrrrrrr}9 & 11 & 8 & 14 & 17 & 14 & 20 & 24 & 17 & 24 & 45 & 60 & 54 & 52 & 48 & 45 & 45 & 38 & 27 & 20 & 8 & 1 & 0 & 0\end{array}$ $\begin{array}{rrrrrrrrrrrrrrrrrrrrrrrrr}0 & 0 & 0 & 0 & 0 & 0 & 0 & 0 & 0 & 0 & 2 & 4 & 13 & 20 & 19 & 29 & 36 & 18 & 0 & 0 & 3 & 4 & 9 & 21 \\ 25 & 23 & 23 & 25 & 26 & 38 & 47 & 42 & 40 & 53 & 53 & 56 & 56 & 54 & 74 & 109 & 139 & 107 & 94 & 70 & 51 & 42 & 54 & 41\end{array}$ 


\begin{tabular}{|c|c|c|c|c|c|c|c|c|c|c|c|c|c|c|c|c|c|c|c|c|c|c|c|}
\hline 58 & 58 & 59 & 45 & 35 & 43 & 35 & 37 & 16 & 17 & 13 & 19 & 23 & 22 & 36 & 34 & 25 & 15 & 14 & 11 & 12 & 9 & 19 & 33 \\
\hline 38 & 36 & 28 & 15 & 17 & 10 & 6 & 3 & 3 & 6 & 15 & 17 & 9 & 10 & 11 & 30 & 39 & 49 & 45 & 9 & 1 & 3 & 13 & 22 \\
\hline 8 & 55 & 42 & 57 & 60 & 59 & 45 & 43 & 37 & 28 & 57 & 65 & 47 & 44 & 67 & 78 & 70 & 68 & 57 & 46 & 60 & 59 & 30 & 38 \\
\hline 29 & 29 & 16 & 11 & 17 & 15 & 17 & 6 & 8 & 24 & 22 & 28 & 17 & 23 & 17 & 15 & 10 & 7 & 4 & 6 & 6 & 16 & 14 & 4 \\
\hline 8 & 22 & 17 & 18 & 23 & 20 & 16 & 27 & 35 & 39 & 37 & 29 & 34 & 29 & 22 & 16 & 25 & 40 & 41 & 29 & 41 & 71 & 84 & 72 \\
\hline 45 & 36 & 34 & 34 & 27 & 14 & 6 & 0 & 2 & 4 & 8 & 8 & 5 & 3 & 1 & 0 & 0 & 2 & 5 & 16 & 9 & 2 & 1 & 3 \\
\hline 8 & 23 & 20 & 43 & 33 & 38 & 27 & 7 & 5 & 4 & 4 & 1 & 1 & 1 & 2 & 1 & 1 & 1 & 1 & 1 & 2 & 2 & 1 & 0 \\
\hline 13 & 38 & 32 & 17 & 11 & 9 & 9 & 2 & 3 & 6 & 6 & 9 & 16 & 18 & 22 & 26 & 24 & 22 & 38 & 46 & 54 & 50 & 45 & 43 \\
\hline 68 & 93 & 96 & 99 & 116 & 107 & 90 & 84 & 105 & 75 & 58 & 43 & 49 & 43 & 39 & 29 & 23 & 13 & 9 & 2 & 4 & 8 & 5 & 8 \\
\hline 3 & 9 & 8 & 9 & 14 & 26 & 21 & 4 & 6 & 16 & 40 & 53 & 69 & 72 & 89 & 98 & 112 & 82 & 48 & 31 & 29 & 50 & 64 & 77 \\
\hline 75 & 64 & 69 & 77 & 84 & 56 & 63 & 46 & 48 & 57 & 88 & 101 & 100 & 94 & 85 & 72 & 70 & 56 & 36 & 35 & 31 & 44 & 49 & 67 \\
\hline 64 & 46 & 40 & 30 & 32 & 9 & 21 & 32 & 28 & 47 & 85 & 81 & 40 & 58 & 63 & 49 & 18 & 25 & 18 & 19 & 23 & 14 & 17 & 28 \\
\hline 48 & 46 & 37 & 30 & 21 & 19 & 15 & 10 & 7 & 13 & 11 & 17 & 19 & 17 & 13 & 12 & 6 & 3 & 0 & 0 & 0 & 1 & 1 & 1 \\
\hline 1 & 2 & 2 & 3 & 5 & 7 & 10 & 5 & 4 & 3 & 2 & 3 & 3 & 1 & 1 & 3 & 5 & 10 & 8 & 3 & 2 & 2 & 4 & 5 \\
\hline 19 & 2 & 4 & 14 & 7 & 7 & 3 & 1 & 1 & 1 & 1 & 4 & 11 & 21 & 8 & 8 & 11 & 15 & 12 & 9 & 4 & 1 & + & 1 \\
\hline 1 & 1 & 1 & 2 & 2 & 2 & 2 & 3 & 11 & 6 & 2 & 8 & 12 & 41 & 59 & 31 & 41 & 40 & 26 & 20 & 15 & 21 & 14 & 5 \\
\hline 20 & 44 & 19 & 25 & 34 & 28 & 38 & 46 & 34 & 16 & 9 & 2 & 3 & 5 & 9 & 13 & 22 & 22 & 26 & 24 & 45 & 67 & 86 & 96 \\
\hline 79 & 66 & 79 & 76 & 77 & 67 & 63 & 60 & 71 & 70 & 79 & 66 & 57 & 68 & 69 & 66 & 66 & 63 & 45 & 48 & 30 & 35 & 50 & 56 \\
\hline 71 & 60 & 81 & 56 & 20 & 0 & 0 & 0 & 0 & 0 & 0 & 0 & 0 & 0 & 0 & 0 & 0 & 0 & 0 & 0 & 0 & 0 & 0 & 0 \\
\hline 0 & 0 & 0 & 0 & 0 & 0 & 0 & 0 & 0 & 0 & 0 & 0 & 0 & 0 & 0 & 0 & 0 & 0 & 1 & 3 & 5 & 21 & 24 & 22 \\
\hline 16 & 14 & 9 & 14 & 19 & 18 & 22 & 23 & 11 & 6 & 10 & 19 & 27 & 44 & 38 & 30 & 19 & 17 & 11 & 20 & 29 & 16 & 31 & 27 \\
\hline 28 & 24 & 10 & 7 & 2 & 2 & 1 & 1 & 1 & 1 & 1 & 1 & 2 & 2 & 1 & 0 & 0 & 0 & 2 & 3 & 10 & 12 & 32 & 40 \\
\hline 46 & 45 & 47 & 47 & 42 & 48 & 28 & 8 & 6 & 6 & 3 & 4 & 11 & 17 & 17 & 16 & 26 & 21 & 22 & 8 & 5 & 2 & 2 & 4 \\
\hline 13 & 9 & 3 & 7 & 3 & 3 & 1 & 1 & 1 & 1 & 3 & 3 & 1 & 1 & 2 & 12 & 1 & 2 & 4 & 9 & 5 & 4 & 13 & 17 \\
\hline 18 & 14 & 11 & 10 & 16 & 18 & 3 & 3 & 3 & 7 & 12 & 19 & 30 & 22 & 17 & 47 & 18 & 21 & 5 & 6 & 2 & 9 & 8 & 9 \\
\hline 16 & 7 & 6 & 9 & 7 & 6 & 1 & 0 & 2 & 2 & 5 & 5 & 9 & 18 & 26 & 10 & 6 & 6 & 3 & 9 & 15 & 20 & 33 & 45 \\
\hline 35 & 59 & 53 & 63 & 83 & 73 & 85 & 111 & 138 & 139 & 109 & 101 & 99 & 107 & 83 & 105 & 90 & 102 & 102 & 70 & 43 & 65 & 96 & 90 \\
\hline 56 & 31 & 24 & 20 & 19 & 28 & 23 & 16 & 3 & 7 & 21 & 24 & 22 & 22 & 20 & 15 & 21 & 28 & 31 & 19 & 17 & 29 & 44 & 58 \\
\hline 53 & 49 & 44 & 41 & 46 & 39 & 16 & 8 & 3 & 1 & 1 & 1 & 0 & 1 & 0 & 0 & 2 & 4 & 8 & 7 & 9 & 6 & 10 & 11 \\
\hline 8 & 4 & 2 & 1 & 0 & 0 & 3 & 6 & 14 & 7 & 2 & 1 & 1 & 1 & 1 & 1 & 1 & 3 & 4 & 11 & 21 & 24 & 20 & 10 \\
\hline 3 & 4 & 3 & 1 & 0 & 0 & 0 & 0 & 0 & 0 & 0 & 0 & 0 & 0 & 0 & 0 & 0 & 1 & 1 & 0 & 0 & 0 & 0 & 0 \\
\hline 0 & 1 & 2 & 3 & 8 & 8 & 6 & 0 & 0 & 0 & 0 & 0 & 0 & 0 & 0 & 0 & 0 & 0 & 0 & 0 & 0 & 0 & 0 & 0 \\
\hline 1 & 1 & 1 & 1 & 0 & 1 & 0 & 0 & 0 & 0 & 0 & 0 & 0 & 1 & 0 & 0 & 2 & 4 & 5 & 1 & 0 & 0 & 0 & 2 \\
\hline 7 & 39 & 51 & 61 & 52 & 32 & 9 & 2 & 3 & 13 & 21 & 29 & 42 & 74 & 107 & 109 & 82 & 50 & 36 & 18 & 29 & 35 & 28 & 26 \\
\hline 26 & 36 & 28 & 25 & 31 & 27 & 12 & 2 & 6 & 8 & 6 & 7 & 16 & 1 & 27 & 108 & 84 & 28 & 26 & 38 & 50 & 74 & 82 & 84 \\
\hline
\end{tabular}


$\begin{array}{lllllllllllllllllllllllll}93 & 106 & 109 & 114 & 115 & 101 & 90 & 111 & 141 & 160 & 166 & 170 & 174 & 174 & 143 & 59 & 134 & 87 & 69 & 53 & 9 & 6 & 3 & 13\end{array}$ $\begin{array}{llllllllllllllllllllllllll}25 & 24 & 22 & 25 & 30 & 38 & 33 & 27 & 30 & 52 & 55 & 65 & 75 & 97 & 98 & 96 & 71 & 57 & 32 & 14 & 14 & 13 & 16 & 25\end{array}$ $\begin{array}{lllllllllllllllllllllllll}26 & 16 & 25 & 48 & 37 & 27 & 26 & 16 & 6 & 0 & 0 & 0 & 0 & 0 & 0 & 0 & 1 & 3 & 7 & 8 & 2 & 4 & 6 & 15\end{array}$ $\begin{array}{rlllllllllllllllllllllll}20 & 10 & 20 & 27 & 38 & 41 & 17 & 7 & 13 & 11 & 9 & 14 & 12 & 11 & 6 & 0 & 0 & 3 & 4 & 17 & 23 & 18 & 16 & 21\end{array}$

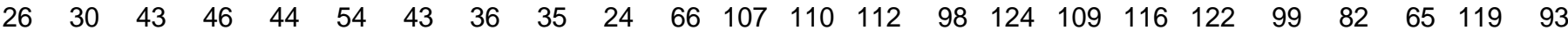
$\begin{array}{lllllllllllllllllllllllll}98 & 69 & 60 & 95 & 90 & 81 & 98 & 109 & 69 & 107 & 96 & 59 & 49 & 42 & 30 & 24 & 24 & 16 & 9 & 7 & 6 & 6 & 9 & 12\end{array}$ $\begin{array}{llllllllllllllllllllllll}17 & 19 & 13 & 14 & 12 & 8 & 7 & 1 & 0 & 0 & 0 & 0 & 0 & 0 & 0 & 0 & 0 & 3 & 7 & 22 & 38 & 46 & 41 & 36\end{array}$ $\begin{array}{llllllllllllllllllllllll}35 & 21 & 10 & 6 & 3 & 5 & 4 & 3 & 2 & 2 & 2 & 5 & 11 & 12 & 15 & 13 & 21 & 15 & 11 & 25 & 39 & 57 & 69 & 71\end{array}$ $\begin{array}{llllllllllllllllllllllll}73 & 73 & 38 & 27 & 26 & 36 & 33 & 19 & 18 & 23 & 15 & 34 & 36 & 71 & 20 & 60 & 27 & 20 & 32 & 31 & 39 & 33 & 29 & 28\end{array}$ $\begin{array}{lllllllllllllllllllllllllll}38 & 41 & 42 & 48 & 52 & 60 & 55 & 105 & 121 & 130 & 138 & 116 & 88 & 66 & 46 & 21 & 51 & 64 & 31 & 21 & 46 & 39 & 37 & 39\end{array}$ $\begin{array}{llllllllllllllllllllllll}33 & 40 & 32 & 37 & 26 & 18 & 18 & 22 & 27 & 21 & 23 & 18 & 12 & 16 & 32 & 25 & 23 & 34 & 44 & 21 & 23 & 23 & 27 & 21\end{array}$ $\begin{array}{lllllllllllllllllllllllllll}17 & 15 & 17 & 24 & 13 & 19 & 22 & 27 & 47 & 33 & 19 & 7 & 5 & 4 & 5 & 13 & 14 & 3 & 0 & 0 & 0 & 0 & 0 & 2\end{array}$ $\begin{array}{llllllllllllllllllllllllll}4 & 7 & 2 & 7 & 9 & 5 & 1 & 2 & 4 & 9 & 10 & 5 & 1 & 5 & 12 & 13 & 15 & 25 & 20 & 15 & 4 & 4 & 5 & 8\end{array}$ $\begin{array}{lllllllllllllllllllllllllll}3 & 5 & 7 & 0 & 1 & 1 & 3 & 7 & 31 & 36 & 37 & 52 & 46 & 64 & 47 & 41 & 38 & 31 & 24 & 20 & 18 & 10 & 10 & 11\end{array}$ $\begin{array}{lllllllllllllllllllllllllll}10 & 10 & 8 & 10 & 17 & 21 & 21 & 4 & 3 & 11 & 35 & 41 & 31 & 107 & 114 & 94 & 81 & 78 & 73 & 42 & 20 & 10 & 36 & 46\end{array}$ $\begin{array}{lllllllllllllllllllllllll}45 & 33 & 29 & 37 & 32 & 32 & 34 & 40 & 43 & 41 & 35 & 41 & 59 & 74 & 89 & 110 & 118 & 117 & 105 & 73 & 65 & 43 & 34 & 36\end{array}$ $\begin{array}{llllllllllllllllllllllllll}36 & 33 & 35 & 38 & 27 & 21 & 27 & 22 & 35 & 41 & 50 & 65 & 71 & 97 & 100 & 104 & 91 & 66 & 33 & 33 & 36 & 30 & 27 & 28\end{array}$ $\begin{array}{llllllllllllllllllllllll}33 & 33 & 28 & 34 & 36 & 29 & 25 & 13 & 7 & 4 & 2 & 2 & 3 & 1 & 3 & 3 & 3 & 9 & 20 & 20 & 21 & 16 & 12 & 15\end{array}$ $\begin{array}{lllllllllllllllllllllllll}20 & 11 & 8 & 10 & 12 & 14 & 19 & 17 & 12 & 19 & 34 & 36 & 38 & 42 & 53 & 54 & 40 & 32 & 39 & 41 & 49 & 56 & 49 & 44\end{array}$ $\begin{array}{llllllllllllllllllllllll}59 & 68 & 66 & 60 & 59 & 80 & 61 & 60 & 82 & 121 & 109 & 125 & 131 & 135 & 107 & 92 & 60 & 54 & 44 & 31 & 10 & 3 & 2 & 3\end{array}$ $\begin{array}{llllllllllllllllllllllll}12 & 4 & 1 & 2 & 3 & 7 & 1 & 0 & 0 & 0 & 2 & 2 & 1 & 2 & 3 & 4 & 4 & 11 & 5 & 8 & 15 & 11 & 17 & 15\end{array}$ $\begin{array}{llllllllllllllllllllllll}22 & 25 & 27 & 32 & 46 & 50 & 35 & 20 & 19 & 15 & 4 & 0 & 1 & 2 & 11 & 33 & 54 & 55 & 44 & 41 & 57 & 69 & 41 & 44\end{array}$ $\begin{array}{rllllllllllllllllllllllll}47 & 60 & 47 & 43 & 30 & 31 & 21 & 13 & 32 & 29 & 26 & 18 & 17 & 16 & 16 & 14 & 15 & 19 & 19 & 18 & 9 & 9 & 12 & 13\end{array}$ $\begin{array}{lllllllllllllllllllllllll}17 & 24 & 29 & 29 & 24 & 9 & 7 & 5 & 2 & 8 & 13 & 21 & 17 & 22 & 28 & 38 & 48 & 15 & 5 & 5 & 14 & 15 & 17 & 23\end{array}$ $\begin{array}{lllllllllllllllllllllllll}45 & 47 & 37 & 33 & 25 & 12 & 9 & 1 & 1 & 2 & 2 & 5 & 12 & 41 & 44 & 44 & 48 & 38 & 23 & 19 & 33 & 41 & 29 & 30\end{array}$ $\begin{array}{llllllllllllllllllllllll}16 & 2 & 5 & 22 & 23 & 40 & 43 & 59 & 79 & 114 & 104 & 98 & 125 & 135 & 141 & 120 & 104 & 110 & 112 & 76 & 74 & 75 & 81 & 84\end{array}$ $\begin{array}{llllllllllllllllllllllllll}71 & 58 & 70 & 62 & 58 & 57 & 59 & 76 & 105 & 123 & 114 & 95 & 78 & 82 & 58 & 37 & 40 & 108 & 63 & 48 & 52 & 69 & 73 & 62\end{array}$ $\begin{array}{llllllllllllllllllllllll}75 & 89 & 76 & 39 & 15 & 11 & 13 & 6 & 15 & 44 & 50 & 44 & 37 & 32 & 30 & 27 & 26 & 24 & 18 & 18 & 27 & 32 & 38 & 34\end{array}$ $\begin{array}{lllllllllllllllllllllllllll}44 & 50 & 50 & 47 & 50 & 31 & 24 & 9 & 0 & 1 & 1 & 2 & 5 & 7 & 3 & 2 & 2 & 2 & 5 & 7 & 12 & 11 & 6 & 3\end{array}$ $\begin{array}{llllllllllllllllllllllllll}1 & 0 & 0 & 0 & 0 & 0 & 0 & 0 & 0 & 1 & 1 & 1 & 0 & 0 & 0 & 0 & 1 & 3 & 15 & 21 & 40 & 32 & 9 & 6\end{array}$ $\begin{array}{llllllllllllllllllllllllllll}9 & 7 & 4 & 2 & 0 & 0 & 0 & 0 & 0 & 0 & 0 & 0 & 1 & 1 & 1 & 1 & 1 & 0 & 2 & 4 & 15 & 6 & 1 & 0\end{array}$ $\begin{array}{lllllllllllllllllllllllll}0 & 0 & 0 & 13 & 20 & 22 & 25 & 27 & 15 & 16 & 11 & 7 & 8 & 10 & 14 & 18 & 21 & 26 & 27 & 25 & 26 & 36 & 60 & 62\end{array}$ $\begin{array}{llllllllllllllllllllllllll}53 & 49 & 55 & 62 & 62 & 46 & 47 & 25 & 12 & 29 & 33 & 35 & 45 & 36 & 37 & 28 & 27 & 24 & 14 & 16 & 27 & 42 & 66 & 73\end{array}$ $\begin{array}{llllllllllllllllllllllll}65 & 62 & 62 & 62 & 75 & 65 & 43 & 17 & 9 & 26 & 65 & 61 & 52 & 58 & 61 & 57 & 59 & 53 & 43 & 58 & 70 & 85 & 111 & 110\end{array}$ $\begin{array}{llllllllllllllllllllllllll}78 & 66 & 62 & 60 & 76 & 77 & 81 & 89 & 26 & 35 & 43 & 42 & 53 & 42 & 36 & 30 & 36 & 23 & 12 & 17 & 24 & 19 & 10 & 7\end{array}$ 
$\begin{array}{rrrrrrrrrrrrrrrrrrrrrrrrr}10 & 15 & 34 & 33 & 36 & 34 & 38 & 19 & 18 & 12 & 8 & 1 & 2 & 2 & 3 & 2 & 3 & 2 & 4 & 7 & 10 & 18 & 26 & 25 \\ 15 & 10 & 9 & 5 & 2 & 1 & 0 & 0 & 0 & 0 & 1 & 1 & 1 & 2 & 1 & 1 & 1 & 2 & 3 & 6 & 7 & 17 & 31 & 47 \\ 42 & 31 & 31 & 33 & 37 & 46 & 40 & 27 & 17 & 29 & 40 & 53 & 51 & 45 & 13 & 11 & 14 & 61 & 118 & 130 & 91 & 69 & 115 & 145 \\ 149 & 139 & 135 & 144 & 163 & 172 & 167 & 167 & 167 & 167 & 167 & 167 & 167 & 50 & 44 & 76 & 61 & 32 & 17 & 20 & 22 & 45 & 32 & 33 \\ 7 & 2 & 2 & 2 & 5 & 2 & 0 & 0 & 0 & 0 & 0 & 2 & 2 & 0 & 2 & 11 & 4 & 1 & 1 & 5 & 1 & 0 & 0 & 2\end{array}$ 


\subsubsection{Validation Data}

The data below is the hourly validation data from Amaranth wind farm used to verify the accuracy of the three models. It is used to generate the plots and tables in section 3.2

\begin{tabular}{|c|c|c|c|c|c|c|c|c|c|c|c|c|c|c|c|c|c|c|c|c|c|c|c|}
\hline \multicolumn{24}{|c|}{ Hour } \\
\hline 1 & 2 & 3 & 4 & 5 & 6 & 7 & 8 & 9 & 10 & 11 & 12 & 13 & 14 & 15 & 16 & 17 & 18 & 19 & 20 & 21 & 22 & 23 & 24 \\
\hline 4 & 6 & 3 & 6 & 12 & 22 & 14 & 9 & 11 & 10 & 19 & 21 & 20 & 19 & 23 & 13 & 14 & 15 & 17 & 23 & 39 & 50 & 38 & 27 \\
\hline 14 & 6 & 4 & 6 & 0 & 0 & 0 & 0 & 0 & 0 & 0 & 0 & 0 & 0 & 0 & 0 & 0 & 0 & 0 & 2 & 5 & 6 & 0 & 0 \\
\hline 2 & 2 & 13 & 43 & 50 & 53 & 54 & 44 & 29 & 36 & 56 & 70 & 107 & 94 & 94 & 94 & 94 & 94 & 31 & 33 & 38 & 38 & 38 & 38 \\
\hline 38 & 38 & 38 & 38 & 38 & 38 & 38 & 38 & 4 & 1 & 0 & 0 & 0 & 0 & 0 & 0 & 0 & 9 & 10 & 11 & 11 & 10 & 12 & 18 \\
\hline 8 & 5 & 5 & 2 & 1 & 5 & 10 & 15 & 26 & 17 & 21 & 26 & 24 & 20 & 30 & 16 & 11 & 10 & 5 & 2 & 9 & 9 & 2 & 8 \\
\hline 6 & 5 & 2 & 2 & 2 & 5 & 6 & 12 & 29 & 50 & 67 & 55 & 62 & 91 & 96 & 113 & 97 & 81 & 74 & 83 & 70 & 103 & 99 & 66 \\
\hline 54 & 50 & 50 & 50 & 44 & 46 & 44 & 36 & 54 & 44 & 49 & 68 & 84 & 134 & 126 & 106 & 103 & 98 & 53 & 61 & 84 & 63 & 36 & 31 \\
\hline 48 & 60 & 71 & 49 & 59 & 83 & 88 & 85 & 80 & 86 & 85 & 78 & 81 & 65 & 57 & 34 & 19 & 34 & 25 & 30 & 37 & 33 & 37 & 47 \\
\hline 33 & 35 & 30 & 37 & 27 & 48 & 42 & 29 & 32 & 27 & 37 & 43 & 43 & 45 & 39 & 31 & 28 & 22 & 19 & 22 & 15 & 12 & 6 & 3 \\
\hline 1 & 0 & 0 & 0 & 1 & 2 & 8 & 19 & 16 & 11 & 10 & 8 & 12 & 14 & 17 & 24 & 24 & 37 & 40 & 38 & 51 & 80 & 101 & 98 \\
\hline 116 & 135 & 108 & 89 & 89 & 88 & 56 & 103 & 72 & 65 & 59 & 49 & 45 & 34 & 49 & 52 & 41 & 39 & 31 & 18 & 17 & 14 & 10 & 11 \\
\hline 22 & 19 & 87 & 108 & 105 & 63 & 45 & 21 & 46 & 46 & 43 & 22 & 11 & 12 & 9 & 2 & 2 & 5 & 7 & 1 & 3 & 6 & 9 & 11 \\
\hline 16 & 22 & 19 & 22 & 36 & 37 & 54 & 38 & 32 & 28 & 10 & 8 & 9 & 6 & 4 & 14 & 16 & 19 & 33 & 69 & 91 & 83 & 54 & 44 \\
\hline 66 & 113 & 136 & 129 & 57 & 35 & 71 & 88 & 89 & 80 & 103 & 105 & 97 & 101 & 111 & 100 & 94 & 99 & 112 & 123 & 131 & 132 & 142 & 99 \\
\hline 102 & 112 & 109 & 92 & 79 & 50 & 30 & 20 & 27 & 70 & 102 & 56 & 62 & 87 & 81 & 61 & 35 & 23 & 20 & 23 & 7 & 6 & 31 & 15 \\
\hline 18 & 38 & 31 & 35 & 28 & 27 & 18 & 23 & 40 & 45 & 95 & 89 & 101 & 115 & 103 & 128 & 136 & 95 & 74 & 61 & 51 & 37 & 43 & 68 \\
\hline 62 & 64 & 61 & 53 & 55 & 57 & 45 & 45 & 45 & 45 & 45 & 45 & 45 & 59 & 72 & 64 & 53 & 40 & 27 & 21 & 24 & 13 & 3 & 3 \\
\hline 5 & 5 & 5 & 10 & 17 & 16 & 11 & 8 & 4 & 13 & 14 & 9 & 8 & 6 & 12 & 19 & 24 & 28 & 29 & 34 & 34 & 39 & 66 & 111 \\
\hline 97 & 47 & 73 & 73 & 71 & 71 & 71 & 57 & 35 & 51 & 35 & 56 & 69 & 57 & 33 & 37 & 34 & 39 & 48 & 53 & 35 & 26 & 31 & 23 \\
\hline 15 & 9 & 12 & 48 & 49 & 55 & 33 & 28 & 44 & 46 & 38 & 28 & 24 & 31 & 29 & 21 & 28 & 25 & 16 & 12 & 10 & 16 & 19 & 16 \\
\hline 18 & 25 & 40 & 39 & 33 & 38 & 27 & 30 & 13 & 10 & 6 & 8 & 6 & 3 & 3 & 6 & 6 & 10 & 21 & 26 & 25 & 25 & 27 & 32 \\
\hline 38 & 47 & 47 & 48 & 56 & 57 & 56 & 41 & 13 & 9 & 11 & 6 & 10 & 11 & 16 & 17 & 19 & 15 & 18 & 37 & 57 & 53 & 50 & 60 \\
\hline 68 & 58 & 74 & 53 & 46 & 40 & 37 & 13 & 12 & 20 & 25 & 27 & 18 & 15 & 13 & 24 & 24 & 21 & 25 & 32 & 37 & 32 & 43 & 56 \\
\hline 53 & 39 & 39 & 49 & 52 & 52 & 59 & 80 & 90 & 94 & 89 & 128 & 112 & 111 & 95 & 111 & 95 & 69 & 47 & 73 & 69 & 48 & 35 & 20 \\
\hline 10 & 22 & 6 & 8 & 25 & 33 & 32 & 16 & 16 & 14 & 26 & 26 & 35 & 46 & 98 & 134 & 137 & 128 & 109 & 102 & 95 & 115 & 102 & 65 \\
\hline
\end{tabular}




$\begin{array}{rrrrrrrrrrrrrrrrrrrrrrrrrrr}40 & 25 & 23 & 32 & 34 & 22 & 31 & 39 & 61 & 57 & 57 & 58 & 48 & 43 & 41 & 38 & 39 & 25 & 11 & 9 & 9 & 8 & 9 & 8 \\ 2 & 0 & 0 & 0 & 0 & 0 & 0 & 1 & 6 & 14 & 9 & 6 & 4 & 4 & 4 & 2 & 1 & 3 & 11 & 11 & 20 & 21 & 18 & 14 \\ 13 & 3 & 0 & 2 & 3 & 3 & 0 & 0 & 0 & 0 & 0 & 0 & 0 & 0 & 0 & 0 & 1 & 2 & 6 & 8 & 4 & 2 & 3 & 12 \\ 10 & 16 & 7 & 9 & 15 & 10 & 9 & 5 & 0 & 0 & 0 & 1 & 2 & 2 & 0 & 0 & 2 & 4 & 5 & 10 & 12 & 9 & 15 & 21 \\ 28 & 25 & 18 & 11 & 6 & 3 & 4 & 1 & 0 & 1 & 2 & 2 & 1 & 1 & 0 & 0 & 0 & 3 & 4 & 4 & 1 & 0 & 2 & 14 \\ 17 & 15 & 13 & 8 & 5 & 7 & 13 & 8 & 1 & 0 & 0 & 0 & 1 & 1 & 0 & 0 & 2 & 3 & 16 & 17 & 18 & 37 & 25 & 26 \\ 34 & 45 & 55 & 53 & 53 & 51 & 37 & 18 & 11 & 11 & 10 & 10 & 12 & 15 & 17 & 27 & 36 & 32 & 54 & 62 & 61 & 71 & 66 & 86 \\ 119 & 106 & 99 & 89 & 84 & 84 & 87 & 65 & 39 & 36 & 27 & 23 & 33 & 39 & 30 & 24 & 23 & 12 & 10 & 10 & 21 & 27 & 25 & 35 \\ 27 & 18 & 15 & 9 & 7 & 7 & 8 & 9 & 8 & 7 & 7 & 1 & 5 & 14 & 33 & 35 & 23 & 18 & 19 & 2 & 0 & 0 & 0 & 3 \\ 6 & 22 & 35 & 46 & 48 & 47 & 36 & 24 & 13 & 9 & 19 & 39 & 56 & 73 & 105 & 116 & 113 & 100 & 105 & 156 & 156 & 160 & 165 & 159\end{array}$




\subsubsection{Wolfe Island Wind Farm Raw Data}

\subsubsection{Training Data}

The data below is the hourly training data from Wolfe Island wind farm used to create the Markov chain and ARMA models. It is used to generate the plots and tables section 3.3.

\begin{tabular}{|c|c|c|c|c|c|c|c|c|c|c|c|c|c|c|c|c|c|c|c|c|c|c|c|}
\hline \multicolumn{24}{|c|}{ Hour } \\
\hline 1 & - & 3 & 4 & 5 & 6 & 7 & 8 & 9 & 10 & 11 & 12 & 13 & 14 & 15 & 16 & 17 & 18 & 19 & 20 & 21 & 22 & 23 & \\
\hline 16 & 23 & 12 & 10 & 8 & 8 & 9 & 12 & 12 & 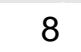 & & & & 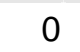 & 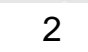 & 4 & 13 & 10 & & & 1 & 30 & 3 & 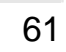 \\
\hline 0 & & & 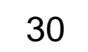 & 22 & 28 & 27 & & & & & & & & & & & & & & & & & \\
\hline 34 & 4 & 78 & 118 & 124 & 135 & 155 & & 165 & 169 & & & 183 & & & & & & & & & 28 & 3 & \\
\hline 6 & 51 & 116 & 111 & 91 & 61 & 59 & 80 & 119 & 108 & 117 & & 133 & 107 & 951 & 115 & 104 & 114 & 117 & 9 & 155 & 158 & & 132 \\
\hline & 301 & 120 & 111 & 116 & 123 & 109 & 119 & 128 & 135 & 145 & & & 115 & 1151 & 122 & 104 & مе & 79 & & & & & 33 \\
\hline 75 & 72 & 83 & 8 & 68 & 70 & 69 & 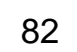 & 82 & 79 & 74 & 50 & 58 & 56 & 40 & 58 & 72 & 100 & 91 & $\perp$ & 3 & OI & 00 & 34 \\
\hline & & & & & & & & 5 & 124 & 118 & 116 & 119 & 125 & 331 & 132 & 136 & 131 & 114 & & & 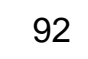 & & 90 \\
\hline 5 & 0 & 36 & 19 & 12 & 1 & 2 & 0 & 36 & 47 & 63 & & & 62 & 2 & 5 & 92 & & 1 & & & 5 & & \\
\hline & & & 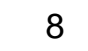 & & 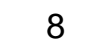 & 6 & & & 2 & 3 & & 12 & 16 & 1 & & 7 & & 3 & 5 & 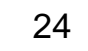 & 40 & 7 & 1 \\
\hline 73 & & & & . & & & & & 74 & 71 & 1 & 51 & 128 & 1 & & 4 & & 7 & 1 & & 17 & & \\
\hline & & & & & & & & & $3 \varepsilon$ & 3 & & 38 & 29 & 35 & & $6 \varepsilon$ & & & & & 30 & & \\
\hline 33 & 19 & 53 & 56 & 46 & 38 & & . & 25 & 15 & 26 & 31 & 1 & 117 & 8 & 30 & 67 & & 4 & 8 & 2 & 29 & 32 & $\angle \perp$ \\
\hline & 6 & 4 & 4 & 7 & 8 & & & 9 & 6 & 8 & 8 & 7 & 2 & & 21 & 27 & 35 & 27 & & & 6 & & 18 \\
\hline & & & & & & & & & 20 & 20 & & & 14 & & & & & 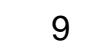 & & & 6 & & \\
\hline 1 & 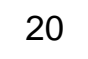 & & & & & & & 5 & 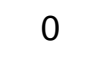 & 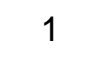 & & & 1 & & & & & 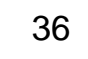 & & & 14 & 101 & \\
\hline 8 & 921 & 104 & 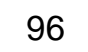 & & 01 & 82 & & & 75 & 82 & & 741 & 101 & 94 & 89 & 69 & 48 & 46 & 2 & & 99 & 62 & 12 \\
\hline 72 & 100 & 121 & 146 & 164 & 144 & 170 & 169 & 183 & 1821 & 1801 & 1821 & 1821 & 1821 & 1751 & 144 & 124 & 146 & 113 & 105 & 9 & 119 & 123 & 120 \\
\hline 24 & 01 & -1 & & 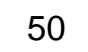 & & 46 & & 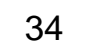 & 47 & 53 & & & 631 & 61 & 177 & 176 & 156 & 124 & 90 & & 13 & 108 & 0 \\
\hline 90 & 71 & 54 & 05 & 84 & 46 & 24 & 24 & $4 \varepsilon$ & & & & & & & & & & $\perp$ & & & 49 & r & \\
\hline & 631 & 127 & 131 & 133 & 104 & 146 & O & 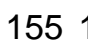 & 39 & & & & 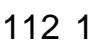 & 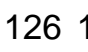 & 23 & 121 & & 99 & & & 8 & & \\
\hline & & 40 & 0 & 35 & 40 & 40 & $0 /$ & & 90 & 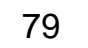 & & & 114 & 96 & 89 & 84 & 76 & 67 & ס & 40 & 24 & 18 & \\
\hline
\end{tabular}


$\begin{array}{llllllllllllllllllllllll}13 & 11 & 16 & 13 & 3 & 0 & 1 & 2 & 10 & 23 & 34 & 45 & 50 & 63 & 68 & 73 & 61 & 80 & 75 & 67 & 128 & 168 & 171 & 143\end{array}$

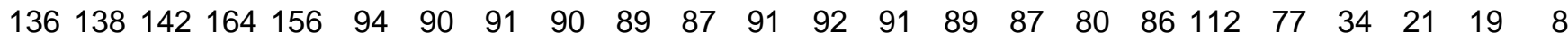
$\begin{array}{llllllllllllllllllllllll}1 & 0 & 0 & 0 & 0 & 2 & 4 & 14 & 25 & 29 & 37 & 52 & 79 & 103 & 55 & 46 & 116 & 148 & 113 & 41 & 54 & 43 & 64 & 102\end{array}$ $\begin{array}{llllllllllllllllllllllll}97 & 101 & 86 & 54 & 54 & 14 & 7 & 1 & 0 & 0 & 1 & 2 & 4 & 2 & 3 & 3 & 2 & 3 & 28 & 32 & 39 & 17 & 38 & 29\end{array}$ $\begin{array}{llllllllllllllllllllllll}17 & 36 & 25 & 24 & 29 & 37 & 27 & 55 & 22 & 83 & 70 & 50 & 33 & 35 & 51 & 88 & 15 & 16 & 23 & 34 & 26 & 83 & 93 & 73\end{array}$ $43 \quad 54 \quad 88112 \quad 96129 \quad 98110108113 \quad 89 \quad 98100146145157150153143162152146 \quad 97 \quad 38$ $\begin{array}{rlllllllllllllllllllllll}10 & 5 & 3 & 2 & 1 & 1 & 0 & 0 & 0 & 0 & 0 & 0 & 0 & 0 & 0 & 0 & 0 & 0 & 0 & 0 & 0 & 0 & 0 & 0\end{array}$

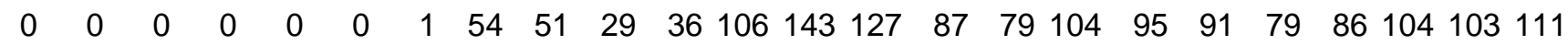
$\begin{array}{llllllllllllllllllllllll}97 & 89 & 57 & 57 & 55 & 40 & 27 & 39 & 56 & 52 & 26 & 18 & 28 & 17 & 15 & 4 & 0 & 2 & 4 & 15 & 42 & 52 & 60 & 68\end{array}$ $\begin{array}{llllllllllllllllllllllll}76 & 96 & 119 & 145 & 141 & 130 & 117 & 92 & 62 & 45 & 31 & 43 & 38 & 56 & 86 & 82 & 31 & 38 & 50 & 81 & 109 & 135 & 161 & 162\end{array}$

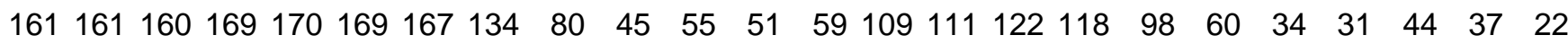
$\begin{array}{llllllllllllllllllllllll}37 & 31 & 20 & 17 & 20 & 16 & 19 & 16 & 32 & 29 & 12 & 5 & 5 & 9 & 14 & 28 & 53 & 44 & 49 & 38 & 47 & 33 & 147 & 135\end{array}$ 152160166158140135137181166121156171176182180182175180167120126124117138 $118 \quad 87 \quad 74 \quad 63 \quad 84134182184184184180167177184182183184184175175175174174162$ $146138126142117125142155156162163162164164159135131 \quad 116 \quad 93 \quad 84 \quad 55 \quad 25 \quad 18 \quad 20$ $\begin{array}{llllllllllllllllllllllll}20 & 16 & 7 & 2 & 1 & 0 & 0 & 0 & 0 & 0 & 0 & 0 & 1 & 1 & 0 & 0 & 1 & 6 & 41 & 69 & 62 & 59 & 62 & 76\end{array}$ $\begin{array}{llllllllllllllllllllllll}68 & 67 & 74 & 88 & 86 & 78 & 50 & 35 & 19 & 13 & 20 & 34 & 43 & 41 & 47 & 51 & 72 & 66 & 59 & 59 & 82 & 101 & 115 & 107\end{array}$ $82104129130124109113 \quad 78 \quad 62 \quad 65 \quad 99122113100 \quad 96110123101 \quad 89 \quad 81 \quad 86114115 \quad 68$

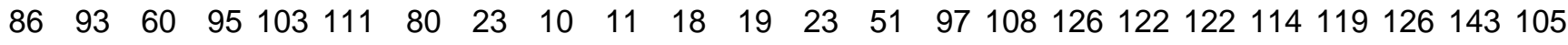
$\begin{array}{llllllllllllllllllllllll}51 & 47 & 52 & 38 & 20 & 14 & 19 & 22 & 26 & 30 & 56 & 127 & 156 & 180 & 179 & 113 & 51 & 63 & 32 & 38 & 56 & 45 & 26 & 35\end{array}$ $\begin{array}{llllllllllllllllllllllll}62 & 71 & 84 & 67 & 45 & 32 & 25 & 5 & 2 & 6 & 7 & 3 & 11 & 14 & 24 & 30 & 38 & 39 & 40 & 54 & 56 & 47 & 50 & 63\end{array}$ $\begin{array}{llllllllllllllllllllllll}86 & 61 & 61 & 69 & 44 & 21 & 35 & 53 & 40 & 28 & 20 & 21 & 31 & 29 & 28 & 35 & 34 & 35 & 26 & 17 & 20 & 31 & 35 & 57\end{array}$ $104126121 \quad 109 \quad 68 \quad 51 \quad 58 \quad 35 \quad 31 \quad 34 \quad 31 \quad 57109146153153143133126108103104 \quad 88 \quad 75$ $\begin{array}{llllllllllllllllllllllll}64 & 50 & 46 & 53 & 52 & 48 & 42 & 43 & 38 & 40 & 32 & 26 & 23 & 19 & 21 & 21 & 21 & 18 & 14 & 17 & 20 & 31 & 24 & 28\end{array}$ $\begin{array}{llllllllllllllllllllllll}20 & 15 & 15 & 22 & 33 & 26 & 21 & 22 & 20 & 28 & 43 & 66 & 72 & 86 & 139 & 167 & 152 & 125 & 80 & 57 & 72 & 70 & 92 & 124\end{array}$

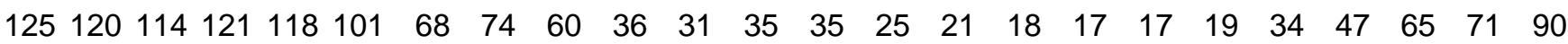
$\begin{array}{llllllllllllllllllllllll}71 & 46 & 29 & 20 & 50 & 64 & 54 & 37 & 40 & 16 & 14 & 24 & 58 & 77 & 68 & 60 & 47 & 36 & 33 & 47 & 58 & 90 & 107 & 112\end{array}$ $\begin{array}{llllllllllllllllllllllll}90 & 72 & 71 & 57 & 54 & 43 & 56 & 51 & 52 & 62 & 57 & 64 & 63 & 75 & 82 & 87 & 89 & 52 & 36 & 48 & 66 & 78 & 80 & 89\end{array}$ $\begin{array}{llllllllllllllllllllllll}85 & 65 & 55 & 71 & 70 & 71 & 53 & 39 & 24 & 7 & 19 & 52 & 78 & 87 & 73 & 59 & 44 & 30 & 19 & 12 & 18 & 32 & 52 & 59\end{array}$ $\begin{array}{llllllllllllllllllllllll}56 & 53 & 43 & 28 & 32 & 42 & 39 & 16 & 10 & 10 & 2 & 4 & 5 & 8 & 10 & 10 & 6 & 24 & 28 & 17 & 9 & 37 & 38 & 32\end{array}$ $\begin{array}{rrrrrrrrrrrrrrrrrrrrrrrr}24 & 27 & 34 & 25 & 17 & 24 & 22 & 4 & 0 & 2 & 3 & 10 & 16 & 16 & 12 & 7 & 9 & 19 & 24 & 19 & 3 & 1 & 2 & 4\end{array}$ $\begin{array}{llllllllllllllllllllllll}12 & 16 & 14 & 9 & 9 & 2 & 2 & 1 & 2 & 4 & 12 & 9 & 5 & 6 & 4 & 9 & 7 & 9 & 9 & 5 & 7 & 11 & 12 & 29\end{array}$ 
$\begin{array}{lllllllllllllllllllllllll}21 & 26 & 33 & 34 & 23 & 24 & 20 & 9 & 1 & 2 & 5 & 11 & 12 & 9 & 14 & 16 & 22 & 15 & 9 & 4 & 3 & 1 & 1 & 1\end{array}$ $\begin{array}{lllllllllllllllllllllllllll}0 & 0 & 3 & 6 & 8 & 4 & 7 & 3 & 1 & 1 & 3 & 9 & 10 & 22 & 8 & 11 & 37 & 22 & 16 & 15 & 15 & 26 & 32 & 45\end{array}$ $\begin{array}{llllllllllllllllllllllll}37 & 49 & 58 & 69 & 66 & 50 & 31 & 43 & 63 & 105 & 151 & 105 & 54 & 28 & 11 & 2 & 4 & 3 & 3 & 5 & 5 & 5 & 14 & 22\end{array}$ $\begin{array}{llllllllllllllllllllllll}26 & 22 & 38 & 55 & 59 & 56 & 64 & 71 & 47 & 36 & 32 & 23 & 10 & 3 & 22 & 34 & 48 & 81 & 73 & 67 & 100 & 157 & 170 & 153\end{array}$ 162140114118129164167177179181183180174178178180181181181177166173166143 $\begin{array}{llllllllllllllllllllllll}101 & 80 & 70 & 76 & 93 & 90 & 72 & 68 & 85 & 126 & 128 & 118 & 137 & 149 & 150 & 150 & 155 & 135 & 102 & 59 & 37 & 29 & 23 & 10\end{array}$

$\begin{array}{llllllllllllllllllllllll}2 & 3 & 15 & 31 & 36 & 34 & 38 & 35 & 56 & 47 & 58 & 71 & 72 & 76 & 64 & 53 & 53 & 51 & 59 & 65 & 72 & 82 & 97 & 109\end{array}$

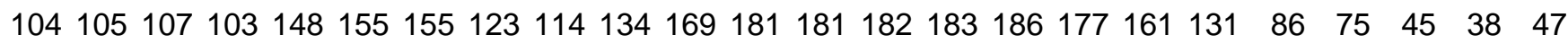
$\begin{array}{llllllllllllllllllllllll}58 & 49 & 72 & 79 & 61 & 38 & 28 & 16 & 32 & 79 & 115 & 122 & 126 & 117 & 109 & 81 & 40 & 33 & 30 & 20 & 21 & 33 & 37 & 25\end{array}$ $\begin{array}{llllllllllllllllllllllll}13 & 8 & 17 & 31 & 40 & 33 & 17 & 11 & 14 & 11 & 4 & 0 & 0 & 0 & 0 & 0 & 2 & 4 & 10 & 7 & 9 & 5 & 5 & 8\end{array}$ $\begin{array}{llllllllllllllllllllllll}14 & 22 & 46 & 52 & 37 & 29 & 21 & 11 & 16 & 32 & 46 & 40 & 35 & 33 & 22 & 20 & 20 & 17 & 18 & 20 & 28 & 35 & 34 & 34\end{array}$ $\begin{array}{llllllllllllllllllllllll}46 & 50 & 44 & 40 & 34 & 34 & 37 & 21 & 20 & 22 & 18 & 30 & 35 & 26 & 25 & 28 & 21 & 21 & 21 & 22 & 33 & 34 & 33 & 46\end{array}$

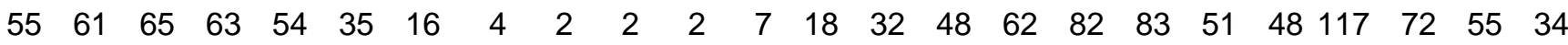
$\begin{array}{llllllllllllllllllllllll}33 & 33 & 42 & 67 & 62 & 46 & 25 & 8 & 9 & 8 & 35 & 65 & 95 & 46 & 1 & 4 & 52 & 68 & 23 & 22 & 19 & 13 & 13 & 26\end{array}$

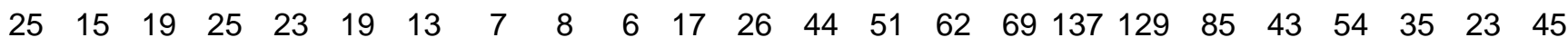
$\begin{array}{lllllllllllllllllllllllll}42 & 31 & 26 & 37 & 54 & 43 & 55 & 65 & 101 & 81 & 33 & 58 & 74 & 102 & 125 & 141 & 135 & 143 & 145 & 151 & 147 & 138 & 128 & 111\end{array}$ $\begin{array}{llllllllllllllllllllllll}111 & 136 & 108 & 95 & 121 & 93 & 106 & 116 & 131 & 133 & 117 & 97 & 96 & 89 & 87 & 83 & 79 & 75 & 63 & 48 & 33 & 31 & 35 & 44\end{array}$ $\begin{array}{llllllllllllllllllllllllll}49 & 42 & 32 & 32 & 42 & 38 & 23 & 25 & 62 & 62 & 71 & 69 & 77 & 79 & 67 & 60 & 70 & 72 & 66 & 45 & 44 & 41 & 40 & 42\end{array}$ $\begin{array}{lllllllllllllllllllllllll}53 & 53 & 41 & 42 & 50 & 35 & 17 & 19 & 57 & 60 & 59 & 61 & 72 & 75 & 72 & 63 & 49 & 31 & 19 & 14 & 19 & 24 & 30 & 39\end{array}$ $\begin{array}{lllllllllllllllllllllllll}41 & 41 & 43 & 26 & 17 & 13 & 10 & 3 & 0 & 0 & 0 & 0 & 0 & 0 & 0 & 1 & 1 & 3 & 3 & 3 & 3 & 2 & 6 & 20\end{array}$ $\begin{array}{llllllllllllllllllllllll}50 & 56 & 52 & 50 & 45 & 55 & 44 & 31 & 43 & 57 & 46 & 39 & 39 & 32 & 24 & 12 & 8 & 51 & 16 & 27 & 19 & 34 & 40 & 34\end{array}$ $\begin{array}{llllllllllllllllllllllll}21 & 64 & 94 & 75 & 67 & 67 & 79 & 89 & 136 & 129 & 103 & 106 & 82 & 65 & 84 & 119 & 109 & 74 & 61 & 44 & 32 & 51 & 63 & 64\end{array}$

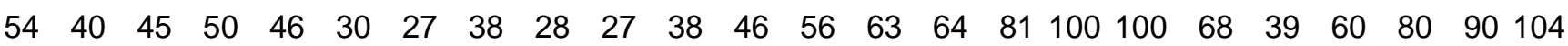
$\begin{array}{llllllllllllllllllllllll}96 & 95 & 103 & 105 & 78 & 58 & 40 & 29 & 27 & 44 & 90 & 98 & 95 & 108 & 149 & 160 & 111 & 75 & 80 & 93 & 59 & 65 & 82 & 40\end{array}$ $\begin{array}{llllllllllllllllllllllll}64 & 114 & 75 & 36 & 33 & 31 & 56 & 57 & 35 & 39 & 62 & 96 & 91 & 64 & 49 & 63 & 78 & 46 & 26 & 34 & 25 & 27 & 36 & 32\end{array}$

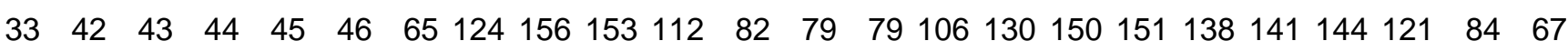
$\begin{array}{llllllllllllllllllllllll}61 & 45 & 36 & 35 & 42 & 62 & 57 & 53 & 58 & 95 & 98 & 108 & 109 & 108 & 113 & 130 & 130 & 122 & 104 & 81 & 43 & 34 & 49 & 67\end{array}$ $\begin{array}{llllllllllllllllllllllll}63 & 52 & 34 & 34 & 26 & 20 & 11 & 15 & 28 & 30 & 26 & 22 & 23 & 19 & 21 & 17 & 14 & 19 & 15 & 12 & 5 & 7 & 5 & 9\end{array}$ $\begin{array}{llllllllllllllllllllllllll}18 & 24 & 23 & 37 & 43 & 37 & 34 & 21 & 12 & 7 & 10 & 18 & 18 & 13 & 17 & 19 & 20 & 20 & 32 & 49 & 62 & 62 & 64 & 72\end{array}$ $\begin{array}{llllllllllllllllllllllllll}55 & 65 & 67 & 73 & 80 & 94 & 77 & 78 & 113 & 135 & 146 & 155 & 150 & 157 & 146 & 120 & 104 & 117 & 124 & 111 & 68 & 49 & 71 & 53\end{array}$ $\begin{array}{llllllllllllllllllllllll}30 & 30 & 21 & 8 & 8 & 6 & 11 & 11 & 13 & 13 & 12 & 8 & 11 & 10 & 15 & 10 & 10 & 11 & 11 & 15 & 20 & 12 & 6 & 3\end{array}$ $\begin{array}{lllllllllllllllllllllllll}2 & 2 & 5 & 9 & 12 & 13 & 9 & 6 & 10 & 6 & 4 & 6 & 7 & 11 & 6 & 5 & 8 & 3 & 2 & 1 & 3 & 4 & 10 & 9\end{array}$ 
$\begin{array}{llllllllllllllllllllllll}8 & 12 & 16 & 14 & 16 & 12 & 3 & 3 & 4 & 2 & 0 & 0 & 0 & 0 & 1 & 2 & 4 & 22 & 38 & 30 & 30 & 35 & 42 & 48\end{array}$ $\begin{array}{llllllllllllllllllllllll}48 & 73 & 88 & 83 & 101 & 96 & 56 & 34 & 16 & 32 & 23 & 40 & 36 & 30 & 33 & 43 & 59 & 80 & 60 & 71 & 49 & 35 & 19 & 11\end{array}$ $\begin{array}{lllllllllllllllllllllllll}9 & 11 & 8 & 14 & 17 & 14 & 20 & 24 & 17 & 24 & 45 & 60 & 54 & 52 & 48 & 45 & 45 & 38 & 27 & 20 & 8 & 1 & 0 & 0\end{array}$ $\begin{array}{llllllllllllllllllllllll}0 & 0 & 0 & 0 & 0 & 0 & 0 & 0 & 0 & 0 & 2 & 4 & 13 & 20 & 19 & 29 & 36 & 18 & 0 & 0 & 3 & 4 & 9 & 21\end{array}$ $\begin{array}{llllllllllllllllllllllll}46 & 36 & 35 & 49 & 51 & 80 & 63 & 46 & 55 & 55 & 67 & 37 & 38 & 41 & 42 & 28 & 14 & 8 & 8 & 3 & 0 & 0 & 1 & 2\end{array}$ $\begin{array}{llllllllllllllllllllllll}3 & 11 & 37 & 43 & 86 & 76 & 56 & 92 & 74 & 41 & 31 & 47 & 58 & 56 & 34 & 29 & 24 & 24 & 24 & 27 & 35 & 27 & 44 & 49\end{array}$

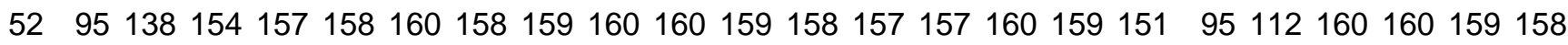
143139159160156158159159159159159160160158159160160156145149155155149116 $\begin{array}{llllllllllllllllllllllll}89 & 63 & 40 & 40 & 31 & 18 & 29 & 19 & 33 & 55 & 55 & 38 & 32 & 14 & 36 & 40 & 75 & 97 & 105 & 94 & 100 & 93 & 94 & 63\end{array}$ $\begin{array}{llllllllllllllllllllllll}45 & 40 & 36 & 40 & 49 & 54 & 66 & 65 & 63 & 61 & 56 & 65 & 79 & 103 & 117 & 87 & 79 & 104 & 112 & 104 & 93 & 96 & 107 & 111\end{array}$ $\begin{array}{llllllllllllllllllllllll}82 & 84 & 88 & 96 & 91 & 74 & 93 & 86 & 63 & 56 & 30 & 17 & 21 & 37 & 43 & 29 & 23 & 10 & 1 & 1 & 3 & 10 & 7 & 3\end{array}$ $\begin{array}{llllllllllllllllllllllll}0 & 0 & 0 & 0 & 1 & 2 & 5 & 9 & 11 & 8 & 2 & 1 & 2 & 4 & 9 & 6 & 1 & 1 & 1 & 11 & 34 & 22 & 24 & 33\end{array}$

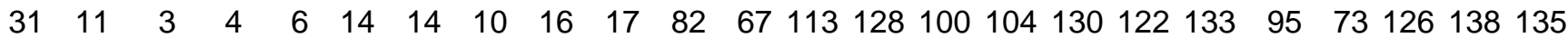
$128139138145154134114103 \quad 99107127135123118116114121130136133101 \quad 87 \quad 75 \quad 71$ $\begin{array}{llllllllllllllllllllllll}89 & 84 & 83 & 95 & 96 & 82 & 67 & 69 & 74 & 78 & 71 & 65 & 52 & 44 & 33 & 33 & 21 & 5 & 16 & 55 & 39 & 23 & 34 & 55\end{array}$ $\begin{array}{llllllllllllllllllllllll}61 & 61 & 66 & 86 & 96 & 82 & 75 & 65 & 77 & 65 & 55 & 64 & 69 & 65 & 72 & 91 & 64 & 43 & 43 & 70 & 68 & 59 & 58 & 44\end{array}$ $\begin{array}{llllllllllllllllllllllll}33 & 39 & 59 & 52 & 53 & 48 & 37 & 31 & 30 & 23 & 20 & 24 & 30 & 20 & 15 & 5 & 1 & 2 & 3 & 4 & 5 & 7 & 4 & 3\end{array}$ $\begin{array}{llllllllllllllllllllllll}16 & 38 & 44 & 41 & 34 & 33 & 36 & 32 & 18 & 16 & 15 & 12 & 7 & 8 & 7 & 6 & 7 & 7 & 12 & 7 & 1 & 0 & 0 & 1\end{array}$ $\begin{array}{llllllllllllllllllllllll}1 & 2 & 6 & 4 & 0 & 0 & 1 & 2 & 5 & 4 & 7 & 18 & 36 & 54 & 46 & 54 & 37 & 22 & 29 & 54 & 77 & 81 & 98 & 85\end{array}$ $\begin{array}{llllllllllllllllllllllll}74 & 85 & 115 & 129 & 124 & 132 & 122 & 80 & 60 & 42 & 42 & 47 & 75 & 102 & 107 & 106 & 92 & 60 & 47 & 64 & 130 & 53 & 54 & 86\end{array}$ $12114914713412312612513311711911610810811111511012613112412613310877 \quad 70$ $\begin{array}{llllllllllllllllllllllll}57 & 52 & 49 & 65 & 70 & 72 & 66 & 49 & 43 & 44 & 78 & 84 & 96 & 97 & 88 & 85 & 90 & 75 & 78 & 84 & 96 & 114 & 134 & 116\end{array}$ $\begin{array}{lllllllllllllllllllllllll}65 & 42 & 23 & 36 & 45 & 44 & 28 & 63 & 98 & 105 & 109 & 117 & 85 & 61 & 42 & 18 & 3 & 1 & 4 & 11 & 27 & 35 & 24 & 36\end{array}$ $\begin{array}{llllllllllllllllllllllll}38 & 26 & 30 & 61 & 74 & 65 & 34 & 23 & 15 & 12 & 22 & 36 & 29 & 37 & 57 & 61 & 48 & 34 & 36 & 57 & 69 & 64 & 59 & 49\end{array}$ $\begin{array}{llllllllllllllllllllllll}38 & 46 & 52 & 50 & 68 & 92 & 59 & 56 & 66 & 88 & 93 & 85 & 80 & 95 & 109 & 83 & 64 & 61 & 46 & 28 & 21 & 6 & 1 & 1\end{array}$ $\begin{array}{llllllllllllllllllllllll}3 & 5 & 9 & 9 & 19 & 24 & 30 & 41 & 58 & 59 & 77 & 72 & 67 & 35 & 52 & 72 & 104 & 112 & 126 & 126 & 99 & 154 & 158 & 160\end{array}$ $161161161161161161161160160160160157151120107 \quad 93 \quad 79 \quad 67 \quad 61 \quad 57 \quad 52 \quad 35 \quad 17 \quad 12$

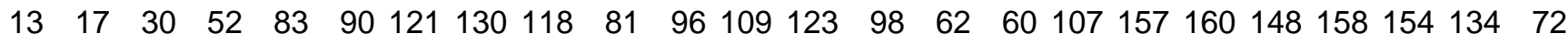
$\begin{array}{llllllllllllllllllllllll}82 & 80 & 45 & 22 & 1 & 2 & 8 & 11 & 10 & 23 & 12 & 10 & 11 & 9 & 11 & 5 & 6 & 21 & 5 & 12 & 16 & 41 & 28 & 28\end{array}$ $\begin{array}{llllllllllllllllllllllll}27 & 17 & 17 & 4 & 5 & 6 & 14 & 55 & 43 & 30 & 27 & 80 & 53 & 52 & 55 & 80 & 139 & 107 & 55 & 53 & 29 & 13 & 14 & 35\end{array}$

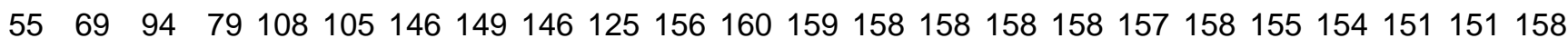

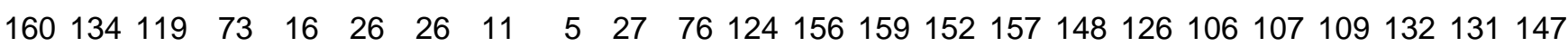




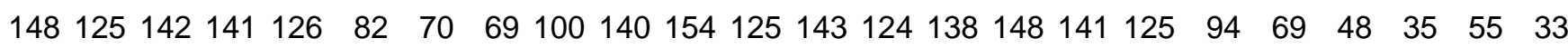
$\begin{array}{llllllllllllllllllllllll}20 & 28 & 25 & 22 & 16 & 18 & 16 & 6 & 1 & 0 & 4 & 2 & 0 & 1 & 5 & 13 & 35 & 43 & 52 & 70 & 90 & 135 & 156 & 158\end{array}$

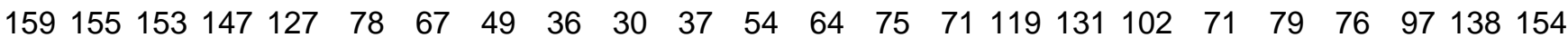
$\begin{array}{llllllllllllllllllllllll}158 & 159 & 156 & 137 & 85 & 63 & 52 & 18 & 26 & 9 & 3 & 3 & 3 & 3 & 3 & 3 & 6 & 4 & 1 & 1 & 11 & 50 & 43 & 42\end{array}$ $\begin{array}{llllllllllllllllllllllll}45 & 72 & 89 & 95 & 99 & 85 & 64 & 49 & 56 & 55 & 37 & 26 & 38 & 34 & 76 & 73 & 96 & 121 & 155 & 156 & 133 & 87 & 37 & 38\end{array}$ $111155155146149151159161158156159159159158156136 \quad 83 \quad 38 \quad 56118128 \quad 99 \quad 23 \quad 4$ 68136115151156155156158153154158158159159159159158158159158156156156155

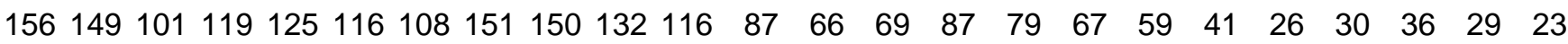
$\begin{array}{llllllllllllllllllllllll}12 & 9 & 12 & 11 & 6 & 7 & 9 & 8 & 11 & 13 & 16 & 18 & 16 & 29 & 43 & 52 & 33 & 38 & 59 & 43 & 40 & 56 & 70 & 97\end{array}$ $\begin{array}{llllllllllllllllllllllll}100 & 82 & 58 & 56 & 52 & 52 & 52 & 26 & 17 & 9 & 7 & 8 & 9 & 14 & 19 & 17 & 16 & 37 & 78 & 117 & 107 & 113 & 122 & 112\end{array}$

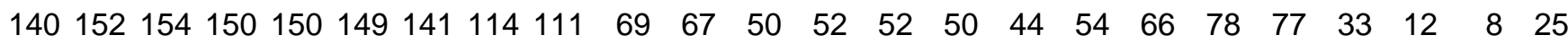
$124154154154115140135135127118 \quad 61100108114 \quad 95100124 \quad 99 \quad 54 \quad 54 \quad 50 \quad 96113114$ $\begin{array}{lllllllllllllllllllllllll}89 & 88 & 76 & 88 & 92 & 74 & 74 & 51 & 98 & 150 & 156 & 155 & 118 & 100 & 145 & 85 & 75 & 36 & 70 & 68 & 38 & 36 & 43 & 40\end{array}$ $\begin{array}{llllllllllllllllllllllll}32 & 31 & 30 & 17 & 7 & 0 & 0 & 0 & 0 & 4 & 11 & 13 & 13 & 25 & 34 & 24 & 22 & 11 & 9 & 45 & 47 & 70 & 76 & 67\end{array}$

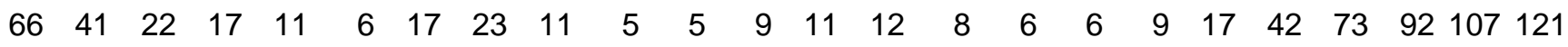
$\begin{array}{llllllllllllllllllllllll}115 & 63 & 50 & 25 & 15 & 20 & 55 & 74 & 82 & 95 & 112 & 98 & 107 & 96 & 94 & 86 & 66 & 48 & 36 & 33 & 55 & 56 & 52 & 67\end{array}$ $\begin{array}{llllllllllllllllllllllll}85 & 64 & 33 & 15 & 7 & 1 & 3 & 5 & 2 & 1 & 6 & 5 & 0 & 3 & 7 & 3 & 16 & 12 & 14 & 29 & 38 & 70 & 68 & 50\end{array}$ $\begin{array}{llllllllllllllllllllllll}49 & 54 & 45 & 51 & 36 & 47 & 52 & 29 & 27 & 24 & 23 & 11 & 6 & 89 & 101 & 76 & 52 & 42 & 32 & 36 & 37 & 49 & 56 & 82\end{array}$ $\begin{array}{llllllllllllllllllllllll}112 & 142 & 130 & 98 & 92 & 91 & 67 & 46 & 41 & 34 & 25 & 14 & 10 & 14 & 16 & 13 & 22 & 13 & 25 & 54 & 63 & 102 & 102 & 104\end{array}$

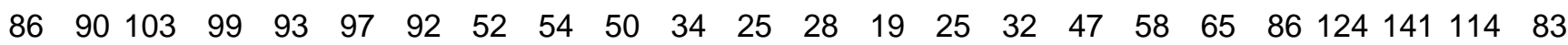

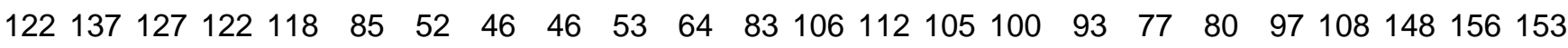
$1461451341171131111116108110 \quad 77 \quad 68 \quad 85 \quad 76 \quad 75 \quad 65 \quad 53 \quad 60 \quad 52 \quad 49 \quad 69111144135115$ $\begin{array}{llllllllllllllllllllllll}110 & 76 & 82 & 90 & 81 & 87 & 86 & 61 & 41 & 37 & 46 & 64 & 76 & 77 & 76 & 59 & 55 & 43 & 33 & 36 & 58 & 57 & 42 & 35\end{array}$ $\begin{array}{llllllllllllllllllllllll}54 & 57 & 54 & 63 & 57 & 56 & 52 & 20 & 25 & 18 & 11 & 15 & 23 & 29 & 26 & 33 & 38 & 28 & 20 & 18 & 34 & 45 & 69 & 88\end{array}$ $\begin{array}{rlllllllllllllllllllllll}78 & 36 & 30 & 37 & 20 & 15 & 15 & 7 & 9 & 23 & 27 & 28 & 51 & 60 & 67 & 66 & 43 & 29 & 10 & 3 & 7 & 3 & 1 & 4\end{array}$ $\begin{array}{llllllllllllllllllllllll}6 & 2 & 10 & 17 & 9 & 8 & 11 & 2 & 0 & 0 & 1 & 0 & 0 & 2 & 2 & 5 & 86 & 9 & 2 & 13 & 13 & 8 & 18 & 28\end{array}$ $\begin{array}{llllllllllllllllllllllll}29 & 22 & 18 & 10 & 0 & 0 & 0 & 0 & 0 & 0 & 0 & 0 & 1 & 2 & 11 & 22 & 10 & 11 & 16 & 32 & 52 & 64 & 73 & 68\end{array}$ $\begin{array}{lllllllllllllllllllllllll}62 & 37 & 2 & 4 & 16 & 36 & 102 & 122 & 129 & 97 & 111 & 134 & 136 & 115 & 110 & 73 & 87 & 79 & 100 & 106 & 114 & 104 & 106 & 76\end{array}$

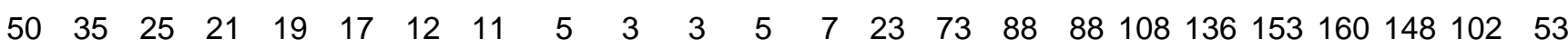

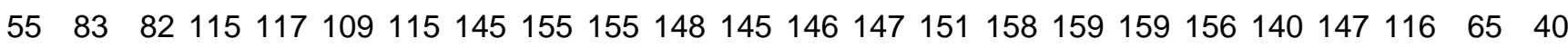
$\begin{array}{llllllllllllllllllllllll}55 & 74 & 87 & 79 & 75 & 50 & 43 & 69 & 36 & 25 & 38 & 18 & 14 & 10 & 5 & 1 & 1 & 1 & 3 & 11 & 28 & 52 & 55 & 58\end{array}$

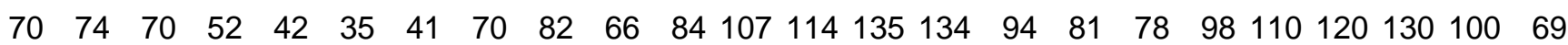


$\begin{array}{llllllllllllllllllllllll}56 & 28 & 64 & 81 & 147 & 152 & 159 & 157 & 135 & 147 & 139 & 133 & 146 & 145 & 123 & 97 & 72 & 40 & 27 & 21 & 36 & 29 & 60 & 79\end{array}$ $\begin{array}{llllllllllllllllllllllll}84 & 32 & 19 & 12 & 7 & 8 & 20 & 12 & 8 & 9 & 23 & 37 & 38 & 28 & 6 & 6 & 15 & 10 & 19 & 43 & 64 & 57 & 38 & 32\end{array}$ $\begin{array}{llllllllllllllllllllllll}43 & 24 & 27 & 22 & 19 & 17 & 15 & 26 & 26 & 17 & 22 & 35 & 45 & 34 & 30 & 40 & 39 & 61 & 52 & 32 & 30 & 56 & 46 & 47\end{array}$ $\begin{array}{llllllllllllllllllllllll}59 & 105 & 90 & 55 & 26 & 12 & 23 & 18 & 25 & 27 & 23 & 31 & 35 & 41 & 50 & 52 & 63 & 62 & 59 & 62 & 76 & 103 & 103 & 93\end{array}$ $\begin{array}{lllllllllllllllllllllllll}77 & 51 & 36 & 38 & 40 & 62 & 49 & 30 & 26 & 15 & 6 & 9 & 22 & 27 & 16 & 12 & 19 & 42 & 37 & 58 & 72 & 103 & 77 & 83\end{array}$ $\begin{array}{llllllllllllllllllllllll}63 & 54 & 45 & 42 & 41 & 31 & 27 & 16 & 17 & 20 & 28 & 27 & 64 & 92 & 118 & 145 & 151 & 140 & 76 & 67 & 71 & 82 & 96 & 95\end{array}$ $\begin{array}{llllllllllllllllllllllll}89 & 82 & 82 & 59 & 67 & 61 & 55 & 57 & 96 & 149 & 119 & 103 & 111 & 86 & 106 & 135 & 142 & 112 & 34 & 9 & 24 & 65 & 11 & 14\end{array}$ $\begin{array}{llllllllllllllllllllllll}56 & 65 & 62 & 88 & 92 & 52 & 81 & 95 & 103 & 79 & 118 & 127 & 121 & 106 & 151 & 158 & 156 & 152 & 89 & 89 & 50 & 44 & 39 & 58\end{array}$

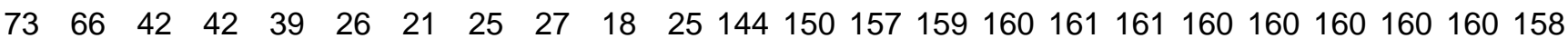

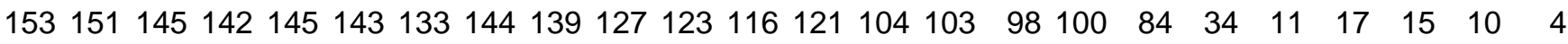

$\begin{array}{llllllllllllllllllllllll}4 & 5 & 1 & 0 & 0 & 0 & 0 & 6 & 9 & 9 & 14 & 28 & 32 & 34 & 45 & 43 & 34 & 65 & 65 & 44 & 52 & 36 & 26 & 20\end{array}$ $\begin{array}{llllllllllllllllllllllll}15 & 11 & 11 & 8 & 5 & 2 & 1 & 1 & 6 & 10 & 13 & 23 & 28 & 17 & 15 & 10 & 6 & 14 & 24 & 25 & 16 & 6 & 4 & 2\end{array}$ $\begin{array}{lllllllllllllllllllllllllll}0 & 1 & 3 & 7 & 9 & 10 & 12 & 6 & 9 & 19 & 24 & 43 & 47 & 27 & 21 & 10 & 19 & 31 & 62 & 82 & 96 & 134 & 137 & 101\end{array}$

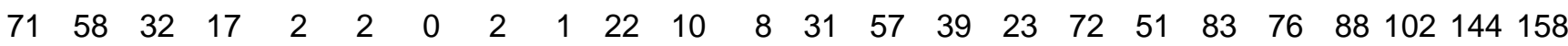
$159158158138 \quad 77 \quad 43 \quad 84113112 \quad 112 \quad 94 \begin{array}{llllllllllllll}132 & 151 & 139 & 87 & 70 & 61 & 43 & 16 & 3 & 0 & 10 & 21 & 28\end{array}$ $\begin{array}{llllllllllllllllllllllll}43 & 53 & 69 & 80 & 73 & 59 & 49 & 43 & 47 & 49 & 53 & 66 & 64 & 72 & 80 & 61 & 64 & 42 & 20 & 74 & 15 & 40 & 64 & 118\end{array}$

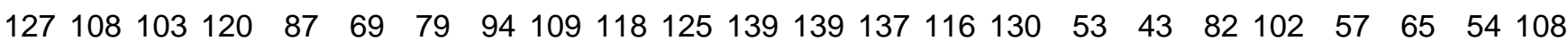
$\begin{array}{llllllllllllllllllllllll}159 & 160 & 154 & 102 & 69 & 49 & 35 & 77 & 116 & 128 & 121 & 133 & 141 & 114 & 129 & 122 & 106 & 56 & 36 & 11 & 13 & 9 & 8 & 29\end{array}$ $\begin{array}{llllllllllllllllllllllll}33 & 23 & 87 & 117 & 108 & 122 & 142 & 154 & 151 & 145 & 140 & 139 & 136 & 124 & 93 & 90 & 71 & 73 & 95 & 100 & 70 & 76 & 71 & 30\end{array}$ $\begin{array}{llllllllllllllllllllllll}30 & 48 & 41 & 27 & 14 & 10 & 6 & 5 & 6 & 6 & 3 & 3 & 5 & 8 & 6 & 3 & 1 & 1 & 1 & 7 & 13 & 15 & 28 & 33\end{array}$ $\begin{array}{llllllllllllllllllllllll}31 & 26 & 19 & 11 & 40 & 54 & 34 & 15 & 4 & 5 & 3 & 4 & 3 & 4 & 9 & 8 & 13 & 22 & 32 & 41 & 36 & 24 & 23 & 24\end{array}$ $\begin{array}{llllllllllllllllllllllll}17 & 12 & 20 & 21 & 23 & 23 & 24 & 8 & 4 & 5 & 17 & 23 & 29 & 31 & 40 & 43 & 48 & 48 & 28 & 9 & 11 & 45 & 51 & 50\end{array}$ $\begin{array}{llllllllllllllllllllllll}44 & 45 & 39 & 32 & 47 & 46 & 39 & 45 & 33 & 27 & 15 & 8 & 31 & 44 & 47 & 42 & 42 & 49 & 59 & 37 & 15 & 17 & 13 & 8\end{array}$ $\begin{array}{llllllllllllllllllllllll}7 & 2 & 10 & 16 & 14 & 20 & 21 & 19 & 15 & 12 & 15 & 12 & 14 & 15 & 22 & 20 & 18 & 14 & 13 & 12 & 10 & 12 & 8 & 6\end{array}$ $\begin{array}{llllllllllllllllllllllll}4 & 3 & 2 & 4 & 4 & 2 & 1 & 0 & 1 & 2 & 1 & 1 & 2 & 0 & 1 & 8 & 8 & 3 & 4 & 2 & 4 & 12 & 12 & 17\end{array}$

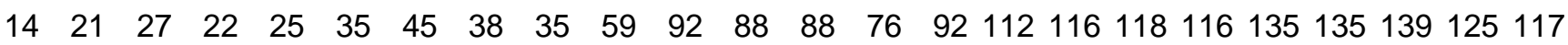
$\begin{array}{llllllllllllllllllllllll}61 & 57 & 49 & 33 & 34 & 47 & 48 & 41 & 36 & 26 & 15 & 4 & 5 & 2 & 3 & 4 & 6 & 1 & 1 & 2 & 5 & 6 & 1 & 2\end{array}$ $\begin{array}{rrrrrrrrrrrrrrrrrrrrrrrr}9 & 12 & 14 & 34 & 41 & 64 & 33 & 29 & 45 & 45 & 45 & 29 & 19 & 11 & 13 & 14 & 17 & 10 & 1 & 1 & 2 & 6 & 3 & 5\end{array}$ $\begin{array}{llllllllllllllllllllllll}10 & 48 & 26 & 3 & 0 & 1 & 1 & 0 & 0 & 1 & 2 & 4 & 38 & 9 & 0 & 1 & 5 & 1 & 5 & 15 & 23 & 54 & 54 & 66\end{array}$ $\begin{array}{llllllllllllllllllllllll}12 & 13 & 14 & 30 & 30 & 35 & 42 & 45 & 36 & 48 & 53 & 48 & 34 & 22 & 18 & 14 & 9 & 7 & 10 & 0 & 0 & 0 & 0 & 0\end{array}$ $\begin{array}{llllllllllllllllllllllll}0 & 1 & 9 & 20 & 51 & 24 & 18 & 25 & 21 & 11 & 6 & 19 & 24 & 19 & 11 & 6 & 5 & 4 & 8 & 8 & 10 & 16 & 27 & 33\end{array}$ $\begin{array}{llllllllllllllllllllllll}55 & 71 & 74 & 75 & 76 & 76 & 76 & 76 & 76 & 76 & 77 & 77 & 77 & 76 & 74 & 76 & 76 & 75 & 70 & 55 & 72 & 74 & 76 & 75\end{array}$ 
$\begin{array}{lllllllllllllllllllllllll}75 & 64 & 68 & 77 & 77 & 75 & 76 & 77 & 77 & 77 & 77 & 76 & 77 & 77 & 76 & 76 & 75 & 75 & 75 & 76 & 77 & 77 & 77 & 76\end{array}$

$\begin{array}{llllllllllllllllllllllll}69 & 58 & 51 & 46 & 29 & 37 & 30 & 30 & 24 & 24 & 24 & 24 & 24 & 24 & 24 & 24 & 24 & 24 & 24 & 24 & 24 & 24 & 24 & 24\end{array}$

$\begin{array}{llllllllllllllllllllllll}24 & 24 & 24 & 24 & 24 & 24 & 24 & 24 & 24 & 24 & 24 & 24 & 24 & 24 & 67 & 67 & 48 & 48 & 61 & 60 & 56 & 55 & 60 & 65\end{array}$ $\begin{array}{lllllllllllllllllllllllll}60 & 49 & 43 & 47 & 59 & 63 & 61 & 50 & 47 & 49 & 34 & 29 & 23 & 18 & 13 & 16 & 15 & 5 & 0 & 3 & 1 & 0 & 0 & 0\end{array}$

$\begin{array}{llllllllllllllllllllllllll}0 & 0 & 1 & 1 & 2 & 3 & 2 & 0 & 0 & 0 & 0 & 0 & 0 & 1 & 0 & 0 & 0 & 0 & 1 & 1 & 3 & 4 & 5 & 12\end{array}$ $\begin{array}{llllllllllllllllllllllll}19 & 5 & 1 & 3 & 3 & 5 & 4 & 6 & 2 & 5 & 33 & 39 & 50 & 51 & 40 & 30 & 39 & 50 & 42 & 43 & 56 & 64 & 70 & 76\end{array}$ $\begin{array}{llllllllllllllllllllllll}76 & 77 & 77 & 77 & 75 & 71 & 72 & 69 & 59 & 35 & 21 & 17 & 11 & 6 & 8 & 1 & 4 & 28 & 60 & 52 & 36 & 27 & 28 & 34\end{array}$ $\begin{array}{llllllllllllllllllllllll}42 & 35 & 40 & 40 & 42 & 37 & 33 & 33 & 37 & 36 & 44 & 49 & 39 & 21 & 11 & 29 & 10 & 5 & 9 & 2 & 1 & 2 & 13 & 17\end{array}$ $\begin{array}{lllllllllllllllllllllllll}23 & 31 & 36 & 40 & 52 & 50 & 47 & 41 & 45 & 50 & 52 & 44 & 46 & 54 & 50 & 42 & 19 & 19 & 22 & 27 & 27 & 8 & 23 & 11\end{array}$ $\begin{array}{lllllllllllllllllllllllll}3 & 0 & 4 & 26 & 41 & 39 & 28 & 19 & 16 & 11 & 11 & 9 & 6 & 5 & 1 & 1 & 1 & 0 & 8 & 8 & 7 & 5 & 9 & 8\end{array}$ $\begin{array}{lllllllllllllllllllllllll}11 & 10 & 13 & 20 & 18 & 16 & 12 & 13 & 9 & 7 & 8 & 12 & 16 & 19 & 14 & 7 & 10 & 11 & 6 & 0 & 0 & 0 & 0 & 1\end{array}$ $\begin{array}{llllllllllllllllllllllll}1 & 2 & 5 & 7 & 3 & 0 & 0 & 1 & 2 & 3 & 6 & 13 & 16 & 20 & 31 & 22 & 18 & 13 & 14 & 19 & 34 & 42 & 49 & 42\end{array}$ $\begin{array}{llllllllllllllllllllllll}47 & 46 & 51 & 64 & 66 & 71 & 73 & 65 & 45 & 24 & 20 & 21 & 28 & 38 & 53 & 63 & 57 & 43 & 50 & 44 & 50 & 32 & 19 & 49\end{array}$ $\begin{array}{lllllllllllllllllllllllllll}22 & 55 & 71 & 60 & 58 & 63 & 65 & 57 & 73 & 72 & 70 & 65 & 64 & 67 & 64 & 61 & 61 & 68 & 66 & 51 & 55 & 52 & 44 & 46\end{array}$ $\begin{array}{lllllllllllllllllllllllllllll}39 & 33 & 26 & 29 & 37 & 23 & 18 & 23 & 34 & 32 & 43 & 57 & 59 & 61 & 59 & 55 & 47 & 46 & 31 & 39 & 28 & 40 & 47 & 53\end{array}$ $\begin{array}{llllllllllllllllllllllll}23 & 16 & 13 & 28 & 34 & 20 & 18 & 24 & 43 & 59 & 65 & 65 & 52 & 42 & 29 & 15 & 5 & 2 & 0 & 1 & 12 & 20 & 14 & 23\end{array}$ $\begin{array}{lllllllllllllllllllllllll}28 & 22 & 20 & 37 & 49 & 46 & 25 & 14 & 11 & 8 & 20 & 23 & 15 & 19 & 24 & 17 & 13 & 9 & 23 & 30 & 30 & 28 & 32 & 37\end{array}$ $\begin{array}{llllllllllllllllllllllllllllll}28 & 29 & 36 & 33 & 33 & 35 & 27 & 27 & 25 & 32 & 33 & 36 & 35 & 36 & 50 & 45 & 37 & 38 & 27 & 15 & 11 & 8 & 4 & 0\end{array}$

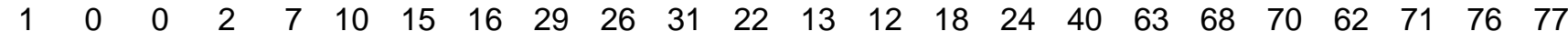




\subsubsection{Validation Data}

The data below is the hourly validation data from Wolfe Island wind farm used to verify the accuracy of the three models. It is used to generate the plots and tables in section 3.3

\begin{tabular}{|c|c|c|c|c|c|c|c|c|c|c|c|c|c|c|c|c|c|c|c|c|c|c|c|}
\hline \multicolumn{24}{|c|}{ Hour } \\
\hline 1 & 2 & 3 & 4 & 5 & 6 & 7 & 8 & 9 & 10 & 11 & 12 & 13 & 14 & 15 & 16 & 17 & 18 & 19 & 20 & 21 & 22 & 23 & 24 \\
\hline 4 & 6 & 3 & 6 & 12 & 22 & 14 & 9 & 11 & 10 & 19 & 21 & 20 & 19 & 23 & 13 & 14 & 15 & 17 & 23 & 39 & 50 & 38 & 27 \\
\hline 14 & 6 & 4 & 6 & 0 & 0 & 0 & 0 & 0 & 0 & 0 & 0 & 0 & 0 & 0 & 0 & 0 & 0 & 0 & 2 & 5 & 6 & 0 & 0 \\
\hline 2 & 2 & 13 & 43 & 50 & 53 & 54 & 44 & 29 & 36 & 56 & 70 & 107 & 94 & 94 & 94 & 94 & 94 & 31 & 33 & 38 & 38 & 38 & 38 \\
\hline 38 & 38 & 38 & 38 & 38 & 38 & 38 & 38 & 4 & 1 & 0 & 0 & 0 & 0 & 0 & 0 & 0 & 9 & 10 & 11 & 11 & 10 & 12 & 18 \\
\hline 8 & 5 & 5 & 2 & 1 & 5 & 10 & 15 & 26 & 17 & 21 & 26 & 24 & 20 & 30 & 16 & 11 & 10 & 5 & 2 & 9 & 9 & 2 & 8 \\
\hline 6 & 5 & 2 & 2 & 2 & 5 & 6 & 12 & 29 & 50 & 67 & 55 & 62 & 91 & 96 & 113 & 97 & 81 & 74 & 83 & 70 & 103 & 99 & 66 \\
\hline 54 & 50 & 50 & 50 & 44 & 46 & 44 & 36 & 54 & 44 & 49 & 68 & 84 & 134 & 126 & 106 & 103 & 98 & 53 & 61 & 84 & 63 & 36 & 31 \\
\hline 48 & 60 & 71 & 49 & 59 & 83 & 88 & 85 & 80 & 86 & 85 & 78 & 81 & 65 & 57 & 34 & 19 & 34 & 25 & 30 & 37 & 33 & 37 & 47 \\
\hline 33 & 35 & 30 & 37 & 27 & 48 & 42 & 29 & 32 & 27 & 37 & 43 & 43 & 45 & 39 & 31 & 28 & 22 & 19 & 22 & 15 & 12 & 6 & 3 \\
\hline 1 & 0 & 0 & 0 & 1 & 2 & 8 & 19 & 16 & 11 & 10 & 8 & 12 & 14 & 17 & 24 & 24 & 37 & 40 & 38 & 51 & 80 & 101 & 98 \\
\hline 116 & 135 & 108 & 89 & 89 & 88 & 56 & 103 & 72 & 65 & 59 & 49 & 45 & 34 & 49 & 52 & 41 & 39 & 31 & 18 & 17 & 14 & 10 & 11 \\
\hline 22 & 19 & 87 & 108 & 105 & 63 & 45 & 21 & 46 & 46 & 43 & 22 & 11 & 12 & 9 & 2 & 2 & 5 & 7 & 1 & 3 & 6 & 9 & 11 \\
\hline 16 & 22 & 19 & 22 & 36 & 37 & 54 & 38 & 32 & 28 & 10 & 8 & 9 & 6 & 4 & 14 & 16 & 19 & 33 & 69 & 91 & 83 & 54 & 44 \\
\hline 66 & 113 & 136 & 129 & 57 & 35 & 71 & 88 & 89 & 80 & 103 & 105 & 97 & 101 & 111 & 100 & 94 & 99 & 112 & 123 & 131 & 132 & 142 & 99 \\
\hline 102 & 112 & 109 & 92 & 79 & 50 & 30 & 20 & 27 & 70 & 102 & 56 & 62 & 87 & 81 & 61 & 35 & 23 & 20 & 23 & 7 & 6 & 31 & 15 \\
\hline 18 & 38 & 31 & 35 & 28 & 27 & 18 & 23 & 40 & 45 & 95 & 89 & 101 & 115 & 103 & 128 & 136 & 95 & 74 & 61 & 51 & 37 & 43 & 68 \\
\hline 62 & 64 & 61 & 53 & 55 & 57 & 45 & 45 & 45 & 45 & 45 & 45 & 45 & 59 & 72 & 64 & 53 & 40 & 27 & 21 & 24 & 13 & 3 & 3 \\
\hline 5 & 5 & 5 & 10 & 17 & 16 & 11 & 8 & 4 & 13 & 14 & 9 & 8 & 6 & 12 & 19 & 24 & 28 & 29 & 34 & 34 & 39 & 66 & 111 \\
\hline 97 & 47 & 73 & 73 & 71 & 71 & 71 & 57 & 35 & 51 & 35 & 56 & 69 & 57 & 33 & 37 & 34 & 39 & 48 & 53 & 35 & 26 & 31 & 23 \\
\hline 15 & 9 & 12 & 48 & 49 & 55 & 33 & 28 & 44 & 46 & 38 & 28 & 24 & 31 & 29 & 21 & 28 & 25 & 16 & 12 & 10 & 16 & 19 & 16 \\
\hline 18 & 25 & 40 & 39 & 33 & 38 & 27 & 30 & 13 & 10 & 6 & 8 & 6 & 3 & 3 & 6 & 6 & 10 & 21 & 26 & 25 & 25 & 27 & 32 \\
\hline 38 & 47 & 47 & 48 & 56 & 57 & 56 & 41 & 13 & 9 & 11 & 6 & 10 & 11 & 16 & 17 & 19 & 15 & 18 & 37 & 57 & 53 & 50 & 60 \\
\hline 68 & 58 & 74 & 53 & 46 & 40 & 37 & 13 & 12 & 20 & 25 & 27 & 18 & 15 & 13 & 24 & 24 & 21 & 25 & 32 & 37 & 32 & 43 & 56 \\
\hline 53 & 39 & 39 & 49 & 52 & 52 & 59 & 80 & 90 & 94 & 89 & 128 & 112 & 111 & 95 & 111 & 95 & 69 & 47 & 73 & 69 & 48 & 35 & 20 \\
\hline
\end{tabular}




$\begin{array}{rrrrrrrrrrrrrrrrrrrrrrrr}10 & 22 & 6 & 8 & 25 & 33 & 32 & 16 & 16 & 14 & 26 & 26 & 35 & 46 & 98 & 134 & 137 & 128 & 109 & 102 & 95 & 115 & 102 & 65 \\ 40 & 25 & 23 & 32 & 34 & 22 & 31 & 39 & 61 & 57 & 57 & 58 & 48 & 43 & 41 & 38 & 39 & 25 & 11 & 9 & 9 & 8 & 9 & 8 \\ 2 & 0 & 0 & 0 & 0 & 0 & 0 & 1 & 6 & 14 & 9 & 6 & 4 & 4 & 4 & 2 & 1 & 3 & 11 & 11 & 20 & 21 & 18 & 14 \\ 13 & 3 & 0 & 2 & 3 & 3 & 0 & 0 & 0 & 0 & 0 & 0 & 0 & 0 & 0 & 0 & 1 & 2 & 6 & 8 & 4 & 2 & 3 & 12 \\ 10 & 16 & 7 & 9 & 15 & 10 & 9 & 5 & 0 & 0 & 0 & 1 & 2 & 2 & 0 & 0 & 2 & 4 & 5 & 10 & 12 & 9 & 15 & 21 \\ 28 & 25 & 18 & 11 & 6 & 3 & 4 & 1 & 0 & 1 & 2 & 2 & 1 & 1 & 0 & 0 & 0 & 3 & 4 & 4 & 1 & 0 & 2 & 14 \\ 17 & 15 & 13 & 8 & 5 & 7 & 13 & 8 & 1 & 0 & 0 & 0 & 1 & 1 & 0 & 0 & 2 & 3 & 16 & 17 & 18 & 37 & 25 & 26 \\ 34 & 45 & 55 & 53 & 53 & 51 & 37 & 18 & 11 & 11 & 10 & 10 & 12 & 15 & 17 & 27 & 36 & 32 & 54 & 62 & 61 & 71 & 66 & 86 \\ 119 & 106 & 99 & 89 & 84 & 84 & 87 & 65 & 39 & 36 & 27 & 23 & 33 & 39 & 30 & 24 & 23 & 12 & 10 & 10 & 21 & 27 & 25 & 35 \\ 27 & 18 & 15 & 9 & 7 & 7 & 8 & 9 & 8 & 7 & 7 & 1 & 5 & 14 & 33 & 35 & 23 & 18 & 19 & 2 & 0 & 0 & 0 & 3 \\ 6 & 22 & 35 & 46 & 48 & 47 & 36 & 24 & 13 & 9 & 19 & 39 & 56 & 73 & 105 & 116 & 113 & 100 & 105 & 156 & 156 & 160 & 165 & 159\end{array}$

\subsubsection{ERCOT System-wide Raw Data}

\subsubsection{Training Data}

The data below is the hourly training data from the ERCOT system-wide used to create the Markov chain and ARMA models.

It is used to generate the plots and tables section 3.4.

\begin{tabular}{|c|c|c|c|c|c|c|c|c|c|c|c|}
\hline \multicolumn{12}{|c|}{ Hour } \\
\hline 1 & 2 & 3 & 4 & 5 & 6 & 7 & 8 & 9 & 10 & 11 & 12 \\
\hline 9554.8 & 9362.31 & 8327 & 7099.26 & 6338.87 & 6003.01 & 5602.71 & 5447.11 & 6474.38 & 6166.71 & 5731.21 & 5305.24 \\
\hline 8727.32 & 8613.33 & 8397.51 & 8040.86 & 7461.66 & 7040.15 & 6351.71 & 6588.31 & 7970.88 & 8545.73 & 8566.86 & 8166.12 \\
\hline 1820.8 & 1976.87 & 2128.83 & 2752.86 & 3436.34 & 3979.16 & 4114.92 & 3830.86 & 2558.99 & 2248.96 & 3501.81 & 4220.43 \\
\hline 679.34 & 781.32 & 1027.15 & 1550.42 & 2030 & 2155.8 & 2110.45 & 1974.87 & 1342.18 & 1181.71 & 1778.51 & 1932.04 \\
\hline 6629.28 & 6385.92 & 5860.43 & 5103.72 & 4372.51 & 3969.99 & 3975.78 & 3674.02 & 2983.4 & 3418.6 & 4185.37 & 4046 \\
\hline 9288.2 & 9275.83 & 9089.71 & 8604.97 & 8054.47 & 6099.93 & 4061.01 & 3097.43 & 2555.08 & 2484.02 & 2177.27 & 2169.81 \\
\hline 4829.18 & 4937.51 & 4716.93 & 3969.75 & 4111.1 & 4153.23 & 4254.91 & 4455.27 & 4650.85 & 5703.12 & 6271.63 & 6016.5 \\
\hline 9119.13 & 9064.24 & 8994.01 & 8980.39 & 8968.66 & 8772.8 & 8607.35 & 8645.82 & 8745.09 & 8958.45 & 8812.39 & 8507.42 \\
\hline
\end{tabular}


$\begin{array}{llllllllllll}9244.11 & 9105.43 & 8956.52 & 8498.43 & 8306.01 & 8207.26 & 8030.05 & 7624.55 & 7392.86 & 7213.8 & 6502.64 & 6386.37\end{array}$ $\begin{array}{lllllllllllll}9002.65 & 8776.63 & 8679.92 & 8421.33 & 7844.11 & 7095.24 & 6296.18 & 6318.25 & 7571.28 & 8112.22 & 7463.71 & 6459.29\end{array}$

\begin{tabular}{rrrrrrrrrrrr} 
& \multicolumn{10}{c}{ Hour } \\
$\mathbf{1 3}$ & $\mathbf{1 4}$ & $\mathbf{1 5}$ & $\mathbf{1 6}$ & $\mathbf{1 7}$ & $\mathbf{1 8}$ & $\mathbf{1 9}$ & $\mathbf{2 0}$ & $\mathbf{2 1}$ & $\mathbf{2 2}$ & $\mathbf{2 3}$ & $\mathbf{2 4}$ \\
5037.53 & 4873.38 & 5139.43 & 4736.39 & 4476.55 & 5263.51 & 5873.18 & 6341.34 & 6914.51 & 7340.4 & 7754.27 & 8311.37 \\
7359.39 & 6355.34 & 5695.04 & 5884.06 & 6697.3 & 7183.5 & 7627.75 & 6149.3 & 3612.71 & 2223.3 & 1813.93 & 1684.56 \\
3808.84 & 3424.41 & 3466.09 & 3681.33 & 3829.01 & 3566.18 & 3162.03 & 2165.47 & 1083.86 & 741.22 & 723.69 & 769.66 \\
1879.01 & 1470.85 & 1467.26 & 1587.05 & 1790.54 & 2076.53 & 2250.42 & 2405.44 & 2586.07 & 4060.31 & 5656.85 & 6599.23 \\
4338.01 & 4510.5 & 4517.47 & 4556.46 & 4588.09 & 4671.75 & 5224.44 & 5398.41 & 6219.67 & 7551 & 8480.46 & 9150.56 \\
2008.73 & 2025.26 & 2011.15 & 2091.31 & 2225.86 & 2442.32 & 2710.15 & 3194.65 & 3733.71 & 4949.77 & 5551.06 & 5382.94 \\
5767.31 & 6053.74 & 6593.25 & 7380.02 & 8147.7 & 8783.21 & 9183.78 & 9226 & 8984.95 & 9024.83 & 9147.5 & 9181.73 \\
8132.72 & 7728.79 & 7452.51 & 7230.17 & 7112.94 & 7392.17 & 7561.07 & 7749.76 & 8018.77 & 8645.5 & 9171.22 & 9284.34 \\
6008.76 & 5452.68 & 5084.97 & 5448.39 & 6110.5 & 6842.59 & 7765.66 & 8351.67 & 8443.56 & 8532.28 & 8794.49 & 9017.56 \\
5632.05 & 5289.15 & 5560.6 & 6210.07 & 6630.9 & 7332.79 & 7696.59 & 7458.72 & 6256.91 & 5999.42 & 6387.87 & 6865.02
\end{tabular}




\subsubsection{Validation Data}

The data below is the hourly validation data from ERCOT system-wide used to verify the accuracy of the three models. It is used to generate the plots and tables in section 3.4

\begin{tabular}{|c|c|c|c|c|c|c|c|c|c|c|c|}
\hline \multicolumn{12}{|c|}{ Hour } \\
\hline 1 & 2 & 3 & 4 & 5 & 6 & 7 & 8 & 9 & 10 & 11 & 12 \\
\hline 6953.88 & 6964.82 & 7560.1 & 7505.61 & 6859.51 & 6468.97 & 5894.84 & 5142.95 & 5158.75 & 5382.66 & 4778.69 & 4119.7 \\
\hline 4629.46 & 4816.23 & 5215.44 & 5214.61 & 4434.27 & 3892.21 & 3425.81 & 2940.78 & 3084.9 & 3718.1 & 4074.4 & 4180.54 \\
\hline \multicolumn{12}{|c|}{ Hour } \\
\hline 13 & 14 & 15 & 16 & 17 & 18 & 19 & 20 & 21 & 22 & 23 & 24 \\
\hline 3718.63 & 3719.3 & 3374.31 & 3338.69 & 3664.04 & 4704.71 & 5844.89 & 5544.6 & 3903.39 & 3566.71 & 4104.15 & 4187.59 \\
\hline 4038.94 & 3762.79 & 3454.73 & 3840.56 & 4904.17 & 5146.76 & 5249.03 & 5245.77 & 5607.97 & 5158.31 & 5275.15 & 4921.21 \\
\hline
\end{tabular}




\subsection{Forecasted Data}

\subsubsection{Amaranth Wind Farm Forecasted Data}

The data below gives the forecasts

produced by each model over the period of

the validation data for Amaranth Wind Farm.

It is used to generate the plots and tables in section 3.2.

Actual Persistence Markov ARMA

$\begin{array}{rrrr}4 & 0 & 4.83 & 3.80 \\ 6 & 4 & 4.50 & 7.04 \\ 3 & 6 & 4.50 & 8.49 \\ 6 & 3 & 4.50 & 4.45 \\ 12 & 6 & 10.00 & 9.20 \\ 22 & 12 & 27.30 & 15.03 \\ 14 & 22 & 20.50 & 25.36 \\ 9 & 14 & 6.17 & 12.75 \\ 11 & 9 & 7.50 & 10.36 \\ 10 & 11 & 10.00 & 13.44 \\ 19 & 10 & 20.90 & 11.34 \\ 21 & 19 & 22.50 & 22.75 \\ 20 & 21 & 21.50 & 21.86 \\ 19 & 20 & 20.90 & 20.90 \\ 23 & 19 & 20.50 & 19.97 \\ 13 & 23 & 11.50 & 25.04 \\ 14 & 13 & 20.50 & 11.55 \\ 15 & 14 & 15.50 & 16.63 \\ 17 & 15 & 21.00 & 16.35 \\ 23 & 17 & 20.50 & 18.82 \\ 39 & 23 & 38.50 & 25.32 \\ 50 & 39 & 46.50 & 42.67 \\ 38 & 50 & 47.50 & 50.89 \\ 27 & 38 & 22.50 & 34.10 \\ 14 & 27 & 20.50 & 25.72 \\ 6 & 14 & 4.50 & 12.50\end{array}$

$\begin{array}{rrrr}4 & 6 & 4.83 & 6.69 \\ 6 & 4 & 4.50 & 5.94 \\ 0 & 6 & 0.00 & 8.55 \\ 0 & 0 & 0.00 & 0.57 \\ 0 & 0 & 0.00 & 2.85 \\ 0 & 0 & 0.00 & 2.17 \\ 0 & 0 & 0.00 & 2.35 \\ 0 & 0 & 0.00 & 2.28 \\ 0 & 0 & 0.00 & 2.29 \\ 0 & 0 & 0.00 & 2.27 \\ 0 & 0 & 0.00 & 2.26 \\ 0 & 0 & 0.00 & 2.24 \\ 0 & 0 & 0.00 & 2.23 \\ 0 & 0 & 0.00 & 2.22 \\ 0 & 0 & 0.00 & 2.20 \\ 0 & 0 & 0.00 & 2.19 \\ 0 & 0 & 0.00 & 2.18 \\ 2 & 0 & 0.00 & 2.17 \\ 5 & 2 & 7.00 & 4.56 \\ 6 & 5 & 4.50 & 7.46 \\ 0 & 6 & 0.00 & 7.81 \\ 0 & 0 & 0.00 & 0.48 \\ 2 & 0 & 0.00 & 2.58 \\ 2 & 2 & 0.00 & 4.36 \\ 13 & 2 & 11.50 & 3.83 \\ 43 & 13 & 39.50 & 17.20 \\ 50 & 43 & 46.50 & 49.40 \\ 53 & 50 & 54.50 & 48.53 \\ 54 & 53 & 44.50 & 52.40 \\ 44 & 54 & 46.50 & 52.50 \\ 29 & 44 & 38.50 & 40.46 \\ 36 & 29 & 36.50 & 25.90 \\ 56 & 36 & 41.50 & 38.51 \\ 70 & 56 & 68.73 & 58.92 \\ 107 & 70 & 99.83 & 69.88 \\ 94 & 107 & 87.94 & 111.22 \\ 94 & 94 & 87.94 & 83.70 \\ 94 & 94 & 87.94 & 91.67 \\ 94 & 94 & 87.94 & 89.40 \\ 94 & 94 & 87.94 & 90.08 \\ 31 & 94 & 25.50 & 89.91 \\ 33 & 31 & 36.17 & 14.25 \\ 38 & 33 & 47.50 & 38.47 \\ 38 & 38 & 47.50 & 37.50\end{array}$




\begin{tabular}{|c|c|c|c|c|c|c|c|}
\hline 38 & 38 & 47.50 & 37.78 & 5 & 10 & 7.00 & 11.05 \\
\hline 38 & 38 & 47.50 & 37.70 & 2 & 5 & 0.00 & 5.37 \\
\hline 38 & 38 & 47.50 & 37.72 & 9 & 2 & 6.17 & 3.39 \\
\hline 38 & 38 & 47.50 & 37.72 & 9 & 9 & 6.17 & 12.37 \\
\hline 38 & 38 & 47.50 & 37.72 & 2 & 9 & 0.00 & 9.77 \\
\hline 38 & 38 & 47.50 & 37.72 & 8 & 2 & 8.50 & 2.09 \\
\hline 38 & 38 & 47.50 & 37.72 & 6 & 8 & 4.50 & 11.50 \\
\hline 38 & 38 & 47.50 & 37.72 & 5 & 6 & 7.00 & 6.38 \\
\hline 38 & 38 & 47.50 & 37.73 & 2 & 5 & 0.00 & 6.64 \\
\hline 38 & 38 & 47.50 & 37.73 & 2 & 2 & 0.00 & 2.95 \\
\hline 4 & 38 & 4.83 & 37.73 & 2 & 2 & 0.00 & 4.00 \\
\hline 1 & 4 & 0.00 & 3.14 & 5 & 2 & 7.00 & 3.68 \\
\hline 0 & 1 & 0.00 & 5.02 & 6 & 5 & 4.50 & 7.37 \\
\hline 0 & 0 & 0.00 & 1.45 & 12 & 6 & 10.00 & 7.49 \\
\hline 0 & 0 & 0.00 & 2.46 & 29 & 12 & 38.50 & 14.66 \\
\hline 0 & 0 & 0.00 & 2.15 & 50 & 29 & 46.50 & 33.02 \\
\hline 0 & 0 & 0.00 & 2.22 & 67 & 50 & 64.50 & 52.97 \\
\hline 0 & 0 & 0.00 & 2.19 & 55 & 67 & 66.50 & 67.67 \\
\hline 0 & 0 & 0.00 & 2.18 & 62 & 55 & 63.50 & 49.02 \\
\hline 9 & 0 & 6.17 & 2.17 & 91 & 62 & 78.28 & 62.83 \\
\hline 10 & 9 & 10.00 & 12.97 & 96 & 91 & 87.83 & 93.72 \\
\hline 11 & 10 & 7.50 & 11.05 & 113 & 96 & 94.50 & 90.85 \\
\hline 11 & 11 & 7.50 & 12.79 & 97 & 113 & 96.40 & 112.15 \\
\hline 10 & 11 & 10.00 & 12.28 & 81 & 97 & 49.50 & 86.81 \\
\hline 12 & 10 & 10.00 & 11.21 & 74 & 81 & 81.37 & 74.92 \\
\hline 18 & 12 & 21.50 & 13.91 & 83 & 74 & 52.50 & 69.96 \\
\hline 8 & 18 & 8.50 & 20.34 & 70 & 83 & 68.73 & 82.23 \\
\hline 5 & 8 & 7.00 & 6.46 & 103 & 70 & 112.50 & 63.09 \\
\hline 5 & 5 & 7.00 & 6.84 & 99 & 103 & 104.00 & 108.30 \\
\hline 2 & 5 & 0.00 & 6.72 & 66 & 99 & 58.50 & 90.48 \\
\hline 1 & 2 & 0.00 & 3.13 & 54 & 66 & 44.50 & 55.98 \\
\hline 5 & 1 & 7.00 & 2.95 & 50 & 54 & 46.50 & 51.52 \\
\hline 10 & 5 & 10.00 & 7.80 & 50 & 50 & 46.50 & 48.01 \\
\hline 15 & 10 & 15.50 & 12.40 & 50 & 50 & 46.50 & 49.03 \\
\hline 26 & 15 & 26.30 & 17.07 & 44 & 50 & 46.50 & 48.74 \\
\hline 17 & 26 & 21.00 & 28.94 & 46 & 44 & 41.00 & 41.62 \\
\hline 21 & 17 & 22.50 & 14.69 & 44 & 46 & 46.50 & 46.08 \\
\hline 26 & 21 & 26.30 & 23.60 & 36 & 44 & 36.50 & 42.40 \\
\hline 24 & 26 & 23.50 & 27.04 & 54 & 36 & 44.50 & 33.85 \\
\hline 20 & 24 & 21.50 & 23.64 & 44 & 54 & 46.50 & 57.95 \\
\hline 30 & 20 & 34.83 & 19.81 & 49 & 44 & 68.50 & 38.99 \\
\hline 16 & 30 & 15.50 & 32.93 & 68 & 49 & 67.00 & 50.47 \\
\hline 11 & 16 & 7.50 & 12.32 & 84 & 68 & 71.00 & 70.01 \\
\hline 10 & 11 & 10.00 & 12.24 & 134 & 84 & 131.00 & 83.63 \\
\hline
\end{tabular}




\begin{tabular}{|c|c|c|c|c|c|c|c|}
\hline 126 & 134 & 126.83 & 139.83 & 37 & 27 & 30.50 & 25.96 \\
\hline 106 & 126 & 117.33 & 114.06 & 43 & 37 & 39.50 & 40.27 \\
\hline 103 & 106 & 112.50 & 97.49 & 43 & 43 & 39.50 & 43.36 \\
\hline 98 & 103 & 109.50 & 98.70 & 45 & 43 & 37.50 & 42.47 \\
\hline 53 & 98 & 54.50 & 92.37 & 39 & 45 & 38.50 & 45.13 \\
\hline 61 & 53 & 53.50 & 40.13 & 31 & 39 & 25.50 & 37.15 \\
\hline 84 & 61 & 71.00 & 64.82 & 28 & 31 & 30.00 & 29.84 \\
\hline 63 & 84 & 54.75 & 85.36 & 22 & 28 & 27.30 & 28.33 \\
\hline 36 & 63 & 36.50 & 54.21 & 19 & 22 & 20.90 & 21.55 \\
\hline 31 & 36 & 25.50 & 30.75 & 22 & 19 & 27.30 & 19.89 \\
\hline 48 & 31 & 47.50 & 31.50 & 15 & 22 & 15.50 & 23.97 \\
\hline 60 & 48 & 60.50 & 51.72 & 12 & 15 & 10.00 & 14.37 \\
\hline 71 & 60 & 67.50 & 60.32 & 6 & 12 & 4.50 & 13.52 \\
\hline 49 & 71 & 68.50 & 71.07 & 3 & 6 & 4.50 & 6.54 \\
\hline 59 & 49 & 81.50 & 41.54 & 1 & 3 & 0.00 & 4.93 \\
\hline 83 & 59 & 52.50 & 62.08 & 0 & 1 & 0.00 & 2.97 \\
\hline 88 & 83 & 78.36 & 85.02 & 0 & 0 & 0.00 & 2.31 \\
\hline 85 & 88 & 112.50 & 84.44 & 0 & 0 & 0.00 & 2.48 \\
\hline 80 & 85 & 95.50 & 81.03 & 1 & 0 & 0.00 & 2.42 \\
\hline 86 & 80 & 93.14 & 76.02 & 2 & 1 & 0.00 & 3.62 \\
\hline 85 & 86 & 112.50 & 84.70 & 8 & 2 & 8.50 & 4.46 \\
\hline 78 & 85 & 73.50 & 81.02 & 19 & 8 & 20.90 & 11.41 \\
\hline 81 & 78 & 49.50 & 73.69 & 16 & 19 & 15.50 & 22.62 \\
\hline 65 & 81 & 67.50 & 79.42 & 11 & 16 & 7.50 & 15.77 \\
\hline 57 & 65 & 60.00 & 58.55 & 10 & 11 & 10.00 & 11.72 \\
\hline 34 & 57 & 36.50 & 54.97 & 8 & 10 & 8.50 & 11.67 \\
\hline 19 & 34 & 20.90 & 28.36 & 12 & 8 & 10.00 & 9.27 \\
\hline 34 & 19 & 36.50 & 18.00 & 14 & 12 & 20.50 & 14.76 \\
\hline 25 & 34 & 22.50 & 39.01 & 17 & 14 & 21.00 & 15.57 \\
\hline 30 & 25 & 34.83 & 22.13 & 24 & 17 & 23.50 & 18.93 \\
\hline 37 & 30 & 30.50 & 33.00 & 24 & 24 & 23.50 & 26.37 \\
\hline 33 & 37 & 36.17 & 38.27 & 37 & 24 & 30.50 & 24.22 \\
\hline 37 & 33 & 30.50 & 31.94 & 40 & 37 & 33.50 & 40.46 \\
\hline 47 & 37 & 51.50 & 38.57 & 38 & 40 & 47.50 & 39.38 \\
\hline 33 & 47 & 36.17 & 48.68 & 51 & 38 & 62.50 & 37.29 \\
\hline 35 & 33 & 39.83 & 28.94 & 80 & 51 & 95.50 & 53.52 \\
\hline 30 & 35 & 34.83 & 37.03 & 101 & 80 & 89.12 & 83.71 \\
\hline 37 & 30 & 30.50 & 28.69 & 98 & 101 & 109.50 & 100.28 \\
\hline 27 & 37 & 22.50 & 39.50 & 116 & 98 & 117.28 & 91.92 \\
\hline 48 & 27 & 47.50 & 24.36 & 135 & 116 & 137.64 & 116.00 \\
\hline 42 & 48 & 52.00 & 53.97 & 108 & 135 & 105.50 & 131.94 \\
\hline 29 & 42 & 38.50 & 38.22 & 89 & 108 & 88.50 & 94.93 \\
\hline 32 & 29 & 20.50 & 27.13 & 89 & 89 & 88.50 & 82.79 \\
\hline 27 & 32 & 22.50 & 33.93 & 88 & 89 & 78.36 & 86.32 \\
\hline
\end{tabular}




\begin{tabular}{|c|c|c|c|c|c|c|c|}
\hline 56 & 88 & 41.50 & 84.12 & 19 & 22 & 20.90 & 24.22 \\
\hline 103 & 56 & 112.50 & 46.31 & 22 & 19 & 27.30 & 18.99 \\
\hline 72 & 103 & 74.25 & 113.72 & 36 & 22 & 36.50 & 24.09 \\
\hline 65 & 72 & 67.50 & 57.05 & 37 & 36 & 30.50 & 39.44 \\
\hline 59 & 65 & 81.50 & 64.99 & 54 & 37 & 44.50 & 36.22 \\
\hline 49 & 59 & 68.50 & 55.50 & 38 & 54 & 47.50 & 57.59 \\
\hline 45 & 49 & 37.50 & 46.23 & 32 & 38 & 20.50 & 32.20 \\
\hline 34 & 45 & 36.50 & 44.10 & 28 & 32 & 30.00 & 32.31 \\
\hline 49 & 34 & 68.50 & 31.49 & 10 & 28 & 10.00 & 27.46 \\
\hline 52 & 49 & 55.75 & 53.15 & 8 & 10 & 8.50 & 7.22 \\
\hline 41 & 52 & 41.50 & 50.52 & 9 & 8 & 6.17 & 10.64 \\
\hline 39 & 41 & 38.50 & 38.06 & 6 & 9 & 4.50 & 10.84 \\
\hline 31 & 39 & 25.50 & 39.25 & 4 & 6 & 4.83 & 7.16 \\
\hline 18 & 31 & 21.50 & 29.29 & 14 & 4 & 20.50 & 5.80 \\
\hline 17 & 18 & 21.00 & 16.53 & 16 & 14 & 15.50 & 18.20 \\
\hline 14 & 17 & 20.50 & 18.99 & 19 & 16 & 20.90 & 17.02 \\
\hline 10 & 14 & 10.00 & 14.66 & 33 & 19 & 36.17 & 20.95 \\
\hline 11 & 10 & 7.50 & 11.09 & 69 & 33 & 63.50 & 36.64 \\
\hline 22 & 11 & 27.30 & 13.31 & 91 & 69 & 78.28 & 75.39 \\
\hline 19 & 22 & 20.90 & 25.88 & 83 & 91 & 52.50 & 90.68 \\
\hline 87 & 19 & 75.00 & 18.64 & 54 & 83 & 44.50 & 76.68 \\
\hline 108 & 87 & 105.50 & 102.46 & 44 & 54 & 46.50 & 45.88 \\
\hline 105 & 108 & 94.41 & 103.56 & 66 & 44 & 58.50 & 42.75 \\
\hline 63 & 105 & 54.75 & 99.67 & 113 & 66 & 94.50 & 70.10 \\
\hline 45 & 63 & 37.50 & 50.33 & 136 & 113 & 128.00 & 118.73 \\
\hline 21 & 45 & 22.50 & 42.93 & 129 & 136 & 124.00 & 132.39 \\
\hline 46 & 21 & 41.00 & 16.22 & 57 & 129 & 60.00 & 120.09 \\
\hline 46 & 46 & 41.00 & 53.97 & 35 & 57 & 39.83 & 37.12 \\
\hline 43 & 46 & 39.50 & 43.08 & 71 & 35 & 67.50 & 34.61 \\
\hline 22 & 43 & 27.30 & 42.62 & 88 & 71 & 78.36 & 78.61 \\
\hline 11 & 22 & 7.50 & 17.51 & 89 & 88 & 88.50 & 86.37 \\
\hline 12 & 11 & 10.00 & 11.52 & 80 & 89 & 95.50 & 85.36 \\
\hline 9 & 12 & 6.17 & 14.43 & 103 & 80 & 112.50 & 74.86 \\
\hline 2 & 9 & 0.00 & 9.97 & 105 & 103 & 94.41 & 105.55 \\
\hline 2 & 2 & 0.00 & 2.83 & 97 & 105 & 96.40 & 99.14 \\
\hline 5 & 2 & 7.00 & 4.87 & 101 & 97 & 89.12 & 91.40 \\
\hline 7 & 5 & 7.00 & 7.87 & 111 & 101 & 106.42 & 98.47 \\
\hline 1 & 7 & 0.00 & 9.39 & 100 & 111 & 110.72 & 108.48 \\
\hline 3 & 1 & 4.50 & 1.72 & 94 & 100 & 87.94 & 92.40 \\
\hline 6 & 3 & 4.50 & 6.32 & 99 & 94 & 104.00 & 89.85 \\
\hline 9 & 6 & 6.17 & 8.58 & 112 & 99 & 83.50 & 96.63 \\
\hline 11 & 9 & 7.50 & 11.52 & 123 & 112 & 115.50 & 110.33 \\
\hline 16 & 11 & 15.50 & 13.06 & 131 & 123 & 134.50 & 119.63 \\
\hline 22 & 16 & 27.30 & 18.62 & 132 & 131 & 135.00 & 126.61 \\
\hline
\end{tabular}




$\begin{array}{rrrr}142 & 132 & 146.83 & 125.84 \\ 99 & 142 & 104.00 & 138.13 \\ 102 & 99 & 92.10 & 82.94 \\ 112 & 102 & 83.50 & 102.48 \\ 109 & 112 & 107.68 & 108.90 \\ 92 & 109 & 84.50 & 103.47 \\ 79 & 92 & 74.00 & 84.63 \\ 50 & 79 & 46.50 & 74.46 \\ 30 & 50 & 34.83 & 42.54 \\ 20 & 30 & 21.50 & 27.70 \\ 27 & 20 & 22.50 & 19.95 \\ 70 & 27 & 68.73 & 30.59 \\ 102 & 70 & 92.10 & 79.21 \\ 56 & 102 & 41.50 & 103.67 \\ 62 & 56 & 63.50 & 41.34 \\ 87 & 62 & 75.00 & 66.53 \\ 81 & 87 & 49.50 & 89.33 \\ 61 & 81 & 53.50 & 75.56 \\ 35 & 61 & 39.83 & 55.50 \\ 23 & 35 & 20.50 & 30.04 \\ 20 & 23 & 21.50 & 22.95 \\ 23 & 20 & 20.50 & 21.37 \\ 7 & 23 & 7.00 & 25.42 \\ 6 & 7 & 4.50 & 5.01 \\ 31 & 6 & 25.50 & 9.67 \\ 15 & 31 & 15.50 & 38.36 \\ 18 & 15 & 21.50 & 10.85 \\ 38 & 18 & 47.50 & 22.37 \\ 31 & 38 & 25.50 & 43.08 \\ 35 & 31 & 39.83 & 28.69 \\ 28 & 35 & 30.00 & 37.64 \\ 27 & 28 & 22.50 & 26.64 \\ 18 & 27 & 21.50 & 28.60 \\ 23 & 18 & 20.50 & 17.20 \\ 40 & 23 & 33.50 & 26.49 \\ 45 & 40 & 37.50 & 44.23 \\ 95 & 45 & 98.00 & 45.12 \\ 89 & 95 & 88.50 & 104.98 \\ 101 & 89 & 89.12 & 80.53 \\ 115 & 115 & 123.50 & 102.02 \\ 103 & 103 & 112.50 & 95.22 \\ 128 & 128.00 & 130.33 \\ 136 & 98.00 & 129.86 \\ 95 & & & \\ & 136 & \end{array}$

$\begin{array}{rrrr}74 & 95 & 81.37 & 80.75 \\ 61 & 74 & 53.50 & 69.69 \\ 51 & 61 & 62.50 & 57.27 \\ 37 & 51 & 30.50 & 48.83 \\ 43 & 37 & 39.50 & 34.44 \\ 68 & 43 & 67.00 & 45.79 \\ 62 & 68 & 63.50 & 72.57 \\ 64 & 62 & 55.50 & 57.65 \\ 61 & 64 & 53.50 & 64.36 \\ 53 & 61 & 54.50 & 58.82 \\ 55 & 53 & 66.50 & 50.81 \\ 57 & 55 & 60.00 & 55.53 \\ 45 & 57 & 37.50 & 56.57 \\ 45 & 45 & 37.50 & 41.85 \\ 45 & 45 & 37.50 & 46.09 \\ 45 & 45 & 37.50 & 44.87 \\ 45 & 45 & 37.50 & 45.22 \\ 45 & 45 & 37.50 & 45.12 \\ 45 & 45 & 37.50 & 45.14 \\ 59 & 45 & 81.50 & 45.13 \\ 72 & 59 & 74.25 & 61.96 \\ 64 & 72 & 55.50 & 72.74 \\ 53 & 64 & 54.50 & 60.03 \\ 40 & 53 & 33.50 & 50.48 \\ 27 & 40 & 22.50 & 37.61 \\ 21 & 27 & 22.50 & 25.69 \\ 24 & 21 & 23.50 & 21.90 \\ 13 & 24 & 11.50 & 26.58 \\ 3 & 13 & 4.50 & 12.00 \\ 3 & 3 & 4.50 & 4.17 \\ 5 & 3 & 7.00 & 6.40 \\ 5 & 5 & 7.00 & 8.14 \\ 5 & 5 & 7.00 & 7.62 \\ 10 & 5 & 10.00 & 7.75 \\ 17 & 10 & 21.00 & 13.70 \\ 16 & 17 & 15.50 & 20.38 \\ 11 & 16 & 7.50 & 17.24 \\ 8 & 11 & 8.50 & 12.12 \\ 4 & 4 & 4.83 & 9.97 \\ 13 & 20.50 & 17.78 \\ 14 & 6.17 & 15.50 \\ 9 & & & \\ 8 & 14.50 & 10.46\end{array}$




\begin{tabular}{|c|c|c|c|c|c|c|c|}
\hline 12 & 6 & 10.00 & 7.94 & 38 & 46 & 47.50 & 45.14 \\
\hline 19 & 12 & 20.90 & 15.86 & 28 & 38 & 30.00 & 36.37 \\
\hline 24 & 19 & 23.50 & 21.97 & 24 & 28 & 23.50 & 26.88 \\
\hline 28 & 24 & 30.00 & 26.21 & 31 & 24 & 25.50 & 24.80 \\
\hline 29 & 28 & 38.50 & 29.79 & 29 & 31 & 38.50 & 33.80 \\
\hline 34 & 29 & 36.50 & 29.95 & 21 & 29 & 22.50 & 28.79 \\
\hline 34 & 34 & 36.50 & 35.91 & 28 & 21 & 30.00 & 20.61 \\
\hline 39 & 34 & 38.50 & 34.18 & 25 & 28 & 22.50 & 31.38 \\
\hline 66 & 39 & 58.50 & 40.69 & 16 & 25 & 15.50 & 24.66 \\
\hline 111 & 66 & 106.42 & 71.27 & 12 & 16 & 10.00 & 15.77 \\
\hline 97 & 111 & 96.40 & 116.56 & 10 & 12 & 10.00 & 13.51 \\
\hline 47 & 97 & 51.50 & 86.70 & 16 & 10 & 15.50 & 11.74 \\
\hline 73 & 47 & 66.50 & 35.23 & 19 & 16 & 20.90 & 19.45 \\
\hline 73 & 73 & 66.50 & 81.33 & 16 & 19 & 15.50 & 20.82 \\
\hline 71 & 73 & 67.50 & 68.05 & 18 & 16 & 21.50 & 16.80 \\
\hline 71 & 71 & 67.50 & 69.48 & 25 & 18 & 22.50 & 20.35 \\
\hline 71 & 71 & 67.50 & 69.08 & 40 & 25 & 33.50 & 27.73 \\
\hline 57 & 71 & 60.00 & 69.21 & 39 & 40 & 38.50 & 43.63 \\
\hline 35 & 57 & 39.83 & 52.36 & 33 & 39 & 36.17 & 37.84 \\
\hline 51 & 35 & 62.50 & 30.77 & 38 & 33 & 47.50 & 32.30 \\
\hline 35 & 51 & 39.83 & 56.23 & 27 & 38 & 22.50 & 39.90 \\
\hline 56 & 35 & 41.50 & 29.65 & 30 & 27 & 34.83 & 24.48 \\
\hline 69 & 56 & 63.50 & 62.56 & 13 & 30 & 11.50 & 32.53 \\
\hline 57 & 69 & 60.00 & 68.70 & 10 & 13 & 10.00 & 9.76 \\
\hline 33 & 57 & 36.17 & 52.52 & 6 & 10 & 4.50 & 12.71 \\
\hline 37 & 33 & 30.50 & 28.34 & 8 & 6 & 8.50 & 7.03 \\
\hline 34 & 37 & 36.50 & 40.11 & 6 & 8 & 4.50 & 11.06 \\
\hline 39 & 34 & 38.50 & 33.10 & 3 & 6 & 4.50 & 7.48 \\
\hline 48 & 39 & 47.50 & 41.13 & 3 & 3 & 4.50 & 4.88 \\
\hline 53 & 48 & 54.50 & 49.63 & 6 & 3 & 4.50 & 5.61 \\
\hline 35 & 53 & 39.83 & 53.19 & 6 & 6 & 4.50 & 8.99 \\
\hline 26 & 35 & 26.30 & 30.53 & 10 & 6 & 10.00 & 8.00 \\
\hline 31 & 26 & 25.50 & 26.24 & 21 & 10 & 22.50 & 13.08 \\
\hline 23 & 31 & 20.50 & 33.48 & 26 & 21 & 26.30 & 24.82 \\
\hline 15 & 23 & 15.50 & 21.77 & 25 & 26 & 22.50 & 27.44 \\
\hline 9 & 15 & 6.17 & 15.52 & 25 & 25 & 22.50 & 25.47 \\
\hline 12 & 9 & 10.00 & 10.09 & 27 & 25 & 22.50 & 26.03 \\
\hline 48 & 12 & 47.50 & 15.24 & 32 & 27 & 20.50 & 28.27 \\
\hline 49 & 48 & 68.50 & 57.02 & 38 & 32 & 47.50 & 33.63 \\
\hline 55 & 49 & 66.50 & 46.18 & 47 & 38 & 51.50 & 39.29 \\
\hline 33 & 55 & 36.17 & 56.52 & 47 & 47 & 51.50 & 48.48 \\
\hline 28 & 33 & 30.00 & 27.09 & 48 & 47 & 47.50 & 45.83 \\
\hline 44 & 28 & 46.50 & 29.56 & 56 & 48 & 41.50 & 47.80 \\
\hline 46 & 44 & 41.00 & 48.08 & 57 & 56 & 60.00 & 56.85 \\
\hline
\end{tabular}




\begin{tabular}{|c|c|c|c|c|c|c|c|}
\hline 56 & 57 & 41.50 & 55.45 & 39 & 39 & 38.50 & 35.53 \\
\hline 41 & 56 & 41.50 & 54.66 & 49 & 39 & 68.50 & 39.82 \\
\hline 13 & 41 & 11.50 & 36.87 & 52 & 49 & 55.75 & 50.60 \\
\hline 9 & 13 & 6.17 & 8.34 & 52 & 52 & 55.75 & 51.11 \\
\hline 11 & 9 & 7.50 & 11.74 & 59 & 52 & 81.50 & 50.97 \\
\hline 6 & 11 & 4.50 & 13.15 & 80 & 59 & 95.50 & 59.43 \\
\hline 10 & 6 & 10.00 & 6.72 & 90 & 80 & 67.83 & 82.24 \\
\hline 11 & 10 & 7.50 & 13.37 & 94 & 90 & 87.94 & 87.71 \\
\hline 16 & 11 & 15.50 & 12.64 & 89 & 94 & 88.50 & 90.96 \\
\hline 17 & 16 & 21.00 & 18.84 & 128 & 89 & 112.50 & 84.04 \\
\hline 19 & 17 & 20.90 & 18.25 & 112 & 128 & 83.50 & 132.95 \\
\hline 15 & 19 & 15.50 & 20.81 & 111 & 112 & 106.42 & 99.65 \\
\hline 18 & 15 & 21.50 & 15.25 & 95 & 111 & 98.00 & 108.08 \\
\hline 37 & 18 & 30.50 & 20.45 & 111 & 95 & 106.42 & 86.45 \\
\hline 57 & 37 & 60.00 & 41.78 & 95 & 111 & 98.00 & 111.95 \\
\hline 53 & 57 & 54.50 & 59.67 & 69 & 95 & 63.50 & 85.40 \\
\hline 50 & 53 & 46.50 & 49.72 & 47 & 69 & 51.50 & 61.82 \\
\hline 60 & 50 & 60.50 & 48.99 & 73 & 47 & 66.50 & 42.19 \\
\hline 68 & 60 & 67.00 & 61.22 & 69 & 73 & 63.50 & 79.11 \\
\hline 58 & 68 & 71.50 & 67.32 & 48 & 69 & 47.50 & 63.67 \\
\hline 74 & 58 & 81.37 & 53.56 & 35 & 48 & 39.83 & 42.89 \\
\hline 53 & 74 & 54.50 & 76.77 & 20 & 35 & 21.50 & 33.25 \\
\hline 46 & 53 & 41.00 & 44.85 & 10 & 20 & 10.00 & 17.99 \\
\hline 40 & 46 & 33.50 & 45.64 & 22 & 10 & 27.30 & 10.36 \\
\hline 37 & 40 & 30.50 & 38.20 & 6 & 22 & 4.50 & 26.97 \\
\hline 13 & 37 & 11.50 & 36.74 & 8 & 6 & 8.50 & 2.94 \\
\hline 12 & 13 & 10.00 & 8.31 & 25 & 8 & 22.50 & 12.26 \\
\hline 20 & 12 & 21.50 & 15.30 & 33 & 25 & 36.17 & 29.99 \\
\hline 25 & 20 & 22.50 & 22.89 & 32 & 33 & 20.50 & 34.48 \\
\hline 27 & 25 & 22.50 & 26.70 & 16 & 32 & 15.50 & 31.98 \\
\hline 18 & 27 & 21.50 & 28.00 & 16 & 16 & 15.50 & 13.46 \\
\hline 15 & 18 & 15.50 & 16.80 & 14 & 16 & 20.50 & 18.79 \\
\hline 13 & 15 & 11.50 & 16.41 & 26 & 14 & 26.30 & 14.84 \\
\hline 24 & 13 & 23.50 & 14.11 & 26 & 26 & 26.30 & 30.39 \\
\hline 24 & 24 & 23.50 & 27.98 & 35 & 26 & 39.83 & 25.90 \\
\hline 21 & 24 & 22.50 & 23.97 & 46 & 35 & 41.00 & 38.01 \\
\hline 25 & 21 & 22.50 & 21.52 & 98 & 46 & 109.50 & 47.74 \\
\hline 32 & 25 & 20.50 & 27.02 & 134 & 98 & 131.00 & 107.45 \\
\hline 37 & 32 & 30.50 & 33.84 & 137 & 134 & 154.50 & 133.53 \\
\hline 32 & 37 & 20.50 & 37.89 & 128 & 137 & 112.50 & 129.66 \\
\hline 43 & 32 & 39.50 & 30.71 & 109 & 128 & 107.68 & 120.00 \\
\hline 56 & 43 & 41.50 & 46.00 & 102 & 109 & 92.10 & 99.99 \\
\hline 53 & 56 & 54.50 & 57.22 & 95 & 102 & 98.00 & 97.38 \\
\hline 39 & 53 & 38.50 & 50.39 & 115 & 95 & 123.50 & 89.74 \\
\hline
\end{tabular}




\begin{tabular}{|c|c|c|c|c|c|c|c|}
\hline 102 & 115 & 92.10 & 116.01 & 11 & 3 & 7.50 & 5.90 \\
\hline 65 & 102 & 67.50 & 92.84 & 11 & 11 & 7.50 & 14.76 \\
\hline 40 & 65 & 33.50 & 55.07 & 20 & 11 & 21.50 & 12.19 \\
\hline 25 & 40 & 22.50 & 35.92 & 21 & 20 & 22.50 & 23.74 \\
\hline 23 & 25 & 20.50 & 23.41 & 18 & 21 & 21.50 & 21.60 \\
\hline 32 & 23 & 20.50 & 24.60 & 14 & 18 & 20.50 & 18.60 \\
\hline 34 & 32 & 36.50 & 35.07 & 13 & 14 & 11.50 & 14.65 \\
\hline 22 & 34 & 27.30 & 34.45 & 3 & 13 & 4.50 & 14.58 \\
\hline 31 & 22 & 25.50 & 20.20 & 0 & 3 & 0.00 & 2.56 \\
\hline 39 & 31 & 38.50 & 35.11 & 2 & 0 & 0.00 & 2.40 \\
\hline 61 & 39 & 53.50 & 40.42 & 3 & 2 & 4.50 & 4.84 \\
\hline 57 & 61 & 60.00 & 65.34 & 3 & 3 & 4.50 & 5.32 \\
\hline 57 & 57 & 60.00 & 53.35 & 0 & 3 & 0.00 & 5.16 \\
\hline 58 & 57 & 71.50 & 56.81 & 0 & 0 & 0.00 & 1.59 \\
\hline 48 & 58 & 47.50 & 57.02 & 0 & 0 & 0.00 & 2.60 \\
\hline 43 & 48 & 39.50 & 44.95 & 0 & 0 & 0.00 & 2.29 \\
\hline 41 & 43 & 41.50 & 42.42 & 0 & 0 & 0.00 & 2.36 \\
\hline 38 & 41 & 47.50 & 40.74 & 0 & 0 & 0.00 & 2.32 \\
\hline 39 & 38 & 38.50 & 37.62 & 0 & 0 & 0.00 & 2.32 \\
\hline 25 & 39 & 22.50 & 39.72 & 0 & 0 & 0.00 & 2.30 \\
\hline 11 & 25 & 7.50 & 22.28 & 0 & 0 & 0.00 & 2.29 \\
\hline 9 & 11 & 6.17 & 10.47 & 0 & 0 & 0.00 & 2.27 \\
\hline 9 & 9 & 6.17 & 11.46 & 1 & 0 & 0.00 & 2.26 \\
\hline 8 & 9 & 8.50 & 11.15 & 2 & 1 & 0.00 & 3.45 \\
\hline 9 & 8 & 6.17 & 10.02 & 6 & 2 & 4.50 & 4.29 \\
\hline 8 & 9 & 8.50 & 11.53 & 8 & 6 & 8.50 & 8.84 \\
\hline 2 & 8 & 0.00 & 9.88 & 4 & 8 & 4.83 & 9.92 \\
\hline 0 & 2 & 0.00 & 3.13 & 2 & 4 & 0.00 & 4.79 \\
\hline 0 & 0 & 0.00 & 2.65 & 3 & 2 & 4.50 & 3.85 \\
\hline 0 & 0 & 0.00 & 2.77 & 12 & 3 & 10.00 & 5.31 \\
\hline 0 & 0 & 0.00 & 2.71 & 10 & 12 & 10.00 & 15.69 \\
\hline 0 & 0 & 0.00 & 2.71 & 16 & 10 & 15.50 & 10.28 \\
\hline 0 & 0 & 0.00 & 2.69 & 7 & 16 & 7.00 & 19.04 \\
\hline 1 & 0 & 0.00 & 2.67 & 9 & 7 & 6.17 & 5.69 \\
\hline 6 & 1 & 4.50 & 3.86 & 15 & 9 & 15.50 & 11.93 \\
\hline 14 & 6 & 20.50 & 9.51 & 10 & 15 & 10.00 & 17.33 \\
\hline 9 & 14 & 6.17 & 17.48 & 9 & 10 & 6.17 & 9.76 \\
\hline 6 & 9 & 4.50 & 9.16 & 5 & 9 & 7.00 & 10.73 \\
\hline 4 & 6 & 4.83 & 7.94 & 0 & 5 & 0.00 & 5.63 \\
\hline 4 & 4 & 4.83 & 5.87 & 0 & 0 & 0.00 & 1.07 \\
\hline 4 & 4 & 4.83 & 6.45 & 0 & 0 & 0.00 & 2.37 \\
\hline 2 & 4 & 0.00 & 6.26 & 1 & 0 & 0.00 & 1.98 \\
\hline 1 & 2 & 0.00 & 3.89 & 2 & 1 & 0.00 & 3.28 \\
\hline 3 & 1 & 4.50 & 3.36 & 2 & 2 & 0.00 & 4.09 \\
\hline
\end{tabular}




\begin{tabular}{|c|c|c|c|c|c|c|c|}
\hline 0 & 2 & 0.00 & 3.84 & 0 & 0 & 0.00 & 1.80 \\
\hline 0 & 0 & 0.00 & 1.50 & 0 & 0 & 0.00 & 1.62 \\
\hline 2 & 0 & 0.00 & 2.16 & 1 & 0 & 0.00 & 1.66 \\
\hline 4 & 2 & 4.83 & 4.36 & 1 & 1 & 0.00 & 2.84 \\
\hline 5 & 4 & 7.00 & 6.11 & 0 & 1 & 0.00 & 2.49 \\
\hline 10 & 5 & 10.00 & 6.80 & 0 & 0 & 0.00 & 1.37 \\
\hline 12 & 10 & 10.00 & 12.60 & 2 & 0 & 0.00 & 1.68 \\
\hline 9 & 12 & 6.17 & 13.32 & 3 & 2 & 4.50 & 3.99 \\
\hline 15 & 9 & 15.50 & 9.50 & 16 & 3 & 15.50 & 4.51 \\
\hline 21 & 15 & 22.50 & 17.80 & 17 & 16 & 21.00 & 19.98 \\
\hline 28 & 21 & 30.00 & 22.61 & 18 & 17 & 21.50 & 16.72 \\
\hline 25 & 28 & 22.50 & 29.63 & 37 & 18 & 30.50 & 18.86 \\
\hline 18 & 25 & 21.50 & 24.00 & 25 & 37 & 22.50 & 41.08 \\
\hline 11 & 18 & 7.50 & 17.21 & 26 & 25 & 26.30 & 20.25 \\
\hline 6 & 11 & 4.50 & 10.75 & 34 & 26 & 36.50 & 27.46 \\
\hline 3 & 6 & 4.50 & 6.59 & 45 & 34 & 37.50 & 35.00 \\
\hline 4 & 3 & 4.83 & 4.17 & 55 & 45 & 66.50 & 46.05 \\
\hline 1 & 4 & 0.00 & 6.06 & 53 & 55 & 54.50 & 54.89 \\
\hline 0 & 1 & 0.00 & 1.89 & 53 & 53 & 54.50 & 49.95 \\
\hline 1 & 0 & 0.00 & 1.88 & 51 & 53 & 62.50 & 51.39 \\
\hline 2 & 1 & 0.00 & 3.07 & 37 & 51 & 30.50 & 48.59 \\
\hline 2 & 2 & 0.00 & 3.92 & 18 & 37 & 21.50 & 32.58 \\
\hline 1 & 2 & 0.00 & 3.66 & 11 & 18 & 7.50 & 14.36 \\
\hline 1 & 1 & 0.00 & 2.52 & 11 & 11 & 7.50 & 11.19 \\
\hline 0 & 1 & 0.00 & 2.83 & 10 & 11 & 10.00 & 12.10 \\
\hline 0 & 0 & 0.00 & 1.53 & 10 & 10 & 10.00 & 10.63 \\
\hline 0 & 0 & 0.00 & 1.89 & 12 & 10 & 10.00 & 11.05 \\
\hline 3 & 0 & 4.50 & 1.77 & 15 & 12 & 15.50 & 13.32 \\
\hline 4 & 3 & 4.83 & 5.40 & 17 & 15 & 21.00 & 16.27 \\
\hline 4 & 4 & 4.83 & 5.54 & 27 & 17 & 22.50 & 17.82 \\
\hline 1 & 4 & 0.00 & 5.49 & 36 & 27 & 36.50 & 29.39 \\
\hline 0 & 1 & 0.00 & 1.89 & 32 & 36 & 20.50 & 36.87 \\
\hline 2 & 0 & 0.00 & 1.71 & 54 & 32 & 44.50 & 29.91 \\
\hline 14 & 2 & 20.50 & 4.15 & 62 & 54 & 63.50 & 58.37 \\
\hline 17 & 14 & 21.00 & 17.86 & 61 & 62 & 53.50 & 59.79 \\
\hline 15 & 17 & 15.50 & 17.51 & 71 & 61 & 67.50 & 58.20 \\
\hline 13 & 15 & 11.50 & 15.20 & 66 & 71 & 58.50 & 70.70 \\
\hline 8 & 13 & 8.50 & 13.46 & 86 & 66 & 93.14 & 61.10 \\
\hline 5 & 8 & 7.00 & 7.94 & 119 & 86 & 118.83 & 87.93 \\
\hline 7 & 5 & 7.00 & 5.92 & 106 & 119 & 117.33 & 119.90 \\
\hline 13 & 7 & 11.50 & 8.90 & 99 & 106 & 104.00 & 95.10 \\
\hline 8 & 13 & 8.50 & 15.24 & 89 & 99 & 88.50 & 93.87 \\
\hline 1 & 8 & 0.00 & 7.39 & 84 & 89 & 71.00 & 82.24 \\
\hline 0 & 1 & 0.00 & 1.23 & 84 & 84 & 71.00 & 79.61 \\
\hline
\end{tabular}




\begin{tabular}{|c|c|c|c|c|c|c|c|}
\hline 87 & 84 & 75.00 & 80.39 & 35 & 22 & 39.83 & 26.38 \\
\hline 65 & 87 & 67.50 & 83.80 & 46 & 35 & 41.00 & 36.68 \\
\hline 39 & 65 & 38.50 & 56.40 & 48 & 46 & 47.50 & 46.94 \\
\hline 36 & 39 & 36.50 & 33.06 & 47 & 48 & 51.50 & 46.39 \\
\hline 27 & 36 & 22.50 & 36.19 & 36 & 47 & 36.50 & 45.36 \\
\hline 23 & 27 & 20.50 & 24.47 & 24 & 36 & 23.50 & 32.44 \\
\hline 33 & 23 & 36.17 & 23.04 & 13 & 24 & 11.50 & 21.74 \\
\hline 39 & 33 & 38.50 & 35.47 & 9 & 13 & 6.17 & 11.60 \\
\hline 30 & 39 & 34.83 & 39.10 & 19 & 9 & 20.90 & 9.71 \\
\hline 24 & 30 & 23.50 & 27.24 & 39 & 19 & 38.50 & 22.27 \\
\hline 23 & 24 & 20.50 & 23.45 & 56 & 39 & 41.50 & 42.69 \\
\hline 12 & 23 & 10.00 & 23.34 & 73 & 56 & 66.50 & 57.24 \\
\hline 10 & 12 & 10.00 & 10.14 & 105 & 73 & 94.41 & 73.50 \\
\hline 10 & 10 & 10.00 & 11.53 & 116 & 105 & 117.28 & 107.30 \\
\hline 21 & 10 & 22.50 & 11.12 & 113 & 116 & 94.50 & 110.81 \\
\hline 27 & 21 & 22.50 & 24.46 & 100 & 113 & 110.72 & 106.24 \\
\hline 25 & 27 & 22.50 & 27.82 & 105 & 100 & 94.41 & 91.97 \\
\hline 35 & 25 & 39.83 & 24.44 & 156 & 105 & 148.83 & 102.13 \\
\hline 27 & 35 & 22.50 & 37.44 & 156 & 156 & 148.83 & 160.55 \\
\hline 18 & 27 & 21.50 & 24.08 & 160 & 156 & 167.50 & 143.76 \\
\hline 15 & 18 & 15.50 & 17.11 & 165 & 160 & 170.50 & 153.47 \\
\hline 9 & 15 & 6.17 & 15.51 & 159 & 165 & 136.50 & 156.75 \\
\hline 7 & 9 & 7.00 & 8.75 & & & & \\
\hline 7 & 7 & 7.00 & 8.28 & & & & \\
\hline 8 & 7 & 8.50 & 8.41 & & & & \\
\hline 9 & 8 & 6.17 & 9.56 & & & & \\
\hline 8 & 9 & 8.50 & 10.42 & & & & \\
\hline 7 & 8 & 7.00 & 8.96 & & & & \\
\hline 7 & 7 & 7.00 & 8.17 & & & & \\
\hline 1 & 7 & 0.00 & 8.39 & & & & \\
\hline 5 & 1 & 7.00 & 1.10 & & & & \\
\hline 14 & 5 & 20.50 & 8.00 & & & & \\
\hline 33 & 14 & 36.17 & 16.82 & & & & \\
\hline 35 & 33 & 39.83 & 37.11 & & & & \\
\hline 23 & 35 & 20.50 & 33.67 & & & & \\
\hline 18 & 23 & 21.50 & 20.24 & & & & \\
\hline 19 & 18 & 20.90 & 18.10 & & & & \\
\hline 2 & 19 & 0.00 & 19.91 & & & & \\
\hline 0 & 2 & 0.00 & 1.05 & & & & \\
\hline 0 & 0 & 0.00 & 2.57 & & & & \\
\hline 0 & 0 & 0.00 & 1.52 & & & & \\
\hline 3 & 0 & 4.50 & 1.81 & & & & \\
\hline 6 & 3 & 4.50 & 5.32 & & & & \\
\hline 22 & 6 & 27.30 & 7.90 & & & & \\
\hline
\end{tabular}




\subsubsection{Wolfe Island Wind Farm Forecasted Data}

The data below gives the forecasts

produced by each model over the period of the validation data for Wolfe Island wind farm. It is used to generate the plots and tables in section 3.3.

$\begin{array}{rrrr}\text { Actual } & \text { Persistence } & \text { Markov } & \text { ARMA } \\ 4 & 0 & 6.50 & 3.79 \\ 6 & 4 & 5.50 & 7.13 \\ 3 & 6 & 4.50 & 8.54 \\ 6 & 3 & 5.50 & 4.46 \\ 12 & 6 & 9.50 & 9.31 \\ 22 & 12 & 21.50 & 15.11 \\ 14 & 22 & 20.50 & 25.48 \\ 9 & 14 & 9.50 & 12.66 \\ 11 & 9 & 7.50 & 10.44 \\ 10 & 11 & 12.50 & 13.52 \\ 19 & 10 & 25.50 & 11.37 \\ 21 & 19 & 21.50 & 22.92 \\ 20 & 21 & 22.50 & 21.86 \\ 19 & 20 & 25.50 & 20.96 \\ 23 & 19 & 22.50 & 20.01 \\ 13 & 23 & 12.50 & 25.14 \\ 14 & 13 & 20.50 & 11.47 \\ 15 & 14 & 13.50 & 16.78 \\ 17 & 15 & 21.50 & 16.38 \\ 23 & 17 & 22.50 & 18.92 \\ 39 & 23 & 37.10 & 25.42 \\ 50 & 39 & 45.50 & 42.86 \\ 38 & 50 & 31.50 & 50.96 \\ 27 & 38 & 29.50 & 33.98 \\ 14 & 27 & 20.50 & 25.75 \\ 6 & 14 & 5.50 & 12.45 \\ 4 & 6 & 6.50 & 6.74 \\ 6 & 4 & 5.50 & 6.02 \\ 0 & 6 & 0.00 & 8.65 \\ 0 & 0 & 0.00 & 0.57 \\ 0 & 0 & 0.00 & 2.99\end{array}$

$\begin{array}{rrr}0 & 0.00 & 2.24 \\ 0 & 0.00 & 2.45 \\ 0 & 0.00 & 2.38 \\ 0 & 0.00 & 2.38 \\ 0 & 0.00 & 2.37 \\ 0 & 0.00 & 2.36 \\ 0 & 0.00 & 2.35 \\ 0 & 0.00 & 2.33 \\ 0 & 0.00 & 2.32 \\ 0 & 0.00 & 2.31 \\ 0 & 0.00 & 2.30 \\ 0 & 0.00 & 2.29 \\ 0 & 5.50 & 2.28 \\ 2 & 10.50 & 4.69 \\ 5 & 5.50 & 7.59 \\ 6 & 0.00 & 7.92 \\ 0 & 0.00 & 0.54 \\ 0 & 5.50 & 2.74 \\ 2 & 5.50 & 4.49 \\ 2 & 12.50 & 3.95 \\ 13 & 44.00 & 17.44 \\ 43 & 45.50 & 49.75 \\ 50 & 50.50 & 48.52 \\ 53 & 51.50 & 52.54 \\ 54 & 50.50 & 52.55 \\ 44 & 38.50 & 40.43 \\ 29 & 34.83 & 25.89 \\ 36 & 53.50 & 38.75 \\ 56 & 77.50 & 59.13 \\ 70 & 107.77 & 69.99 \\ 107 & 91.57 & 111.60 \\ 94 & 91.57 & 83.35 \\ 94 & 91.57 & 91.87 \\ 94 & 91.57 & 89.33 \\ 94 & 91.57 & 90.12 \\ 94 & 36.50 & 89.91 \\ 31 & 32.50 & 13.61 \\ 33 & 31.50 & 38.98 \\ 38 & 31.50 & 37.41 \\ 38 & 31.50 & 37.88 \\ 38 & 31.50 & 37.74 \\ 38 & 31.50 & 37.79 \\ 38 & 31.50 & 37.77 \\ 38 & 31.50 & 37.78 \\ 38 & 31.50 & 37.78 \\ 38 & 31.50 & 37.78 \\ 38 & 31.50 & 37.78 \\ 38 & 31.50 & 37.78\end{array}$




\begin{tabular}{|c|c|c|c|c|c|c|c|}
\hline 38 & 38 & 31.50 & 37.78 & 12 & 6 & 9.50 & 7.62 \\
\hline 4 & 38 & 6.50 & 37.78 & 29 & 12 & 38.50 & 14.86 \\
\hline 1 & 4 & 0.00 & -3.44 & 50 & 29 & 45.50 & 33.29 \\
\hline 0 & 1 & 0.00 & 5.30 & 67 & 50 & 62.50 & 53.21 \\
\hline 0 & 0 & 0.00 & 1.45 & 55 & 67 & 72.50 & 67.84 \\
\hline 0 & 0 & 0.00 & 2.59 & 62 & 55 & 78.00 & 48.90 \\
\hline 0 & 0 & 0.00 & 2.23 & 91 & 62 & 65.50 & 63.09 \\
\hline 0 & 0 & 0.00 & 2.33 & 96 & 91 & 92.50 & 94.00 \\
\hline 0 & 0 & 0.00 & 2.28 & 113 & 96 & 103.21 & 90.79 \\
\hline 0 & 0 & 0.00 & 2.28 & 97 & 113 & 109.50 & 112.40 \\
\hline 9 & 0 & 9.50 & 2.27 & 81 & 97 & 83.03 & 86.53 \\
\hline 10 & 9 & 12.50 & 13.17 & 74 & 81 & 77.50 & 74.94 \\
\hline 11 & 10 & 7.50 & 11.10 & 83 & 74 & 52.50 & 69.96 \\
\hline 11 & 11 & 7.50 & 12.92 & 70 & 83 & 77.50 & 82.39 \\
\hline 10 & 11 & 12.50 & 12.36 & 103 & 70 & 112.50 & 62.91 \\
\hline 12 & 10 & 9.50 & 11.31 & 99 & 103 & 93.28 & 108.79 \\
\hline 18 & 12 & 15.83 & 14.04 & 66 & 99 & 79.50 & 90.17 \\
\hline 8 & 18 & 9.50 & 20.49 & 54 & 66 & 51.50 & 55.79 \\
\hline 5 & 8 & 10.50 & 6.42 & 50 & 54 & 45.50 & 51.59 \\
\hline 5 & 5 & 10.50 & 7.00 & 50 & 50 & 45.50 & 48.01 \\
\hline 2 & 5 & 5.50 & 6.81 & 50 & 50 & 45.50 & 49.09 \\
\hline 1 & 2 & 0.00 & 3.22 & 44 & 50 & 50.50 & 48.77 \\
\hline 5 & 1 & 10.50 & 3.08 & 46 & 44 & 47.00 & 41.60 \\
\hline 10 & 5 & 12.50 & 7.96 & 44 & 46 & 50.50 & 46.19 \\
\hline 15 & 10 & 13.50 & 12.54 & 36 & 44 & 34.83 & 42.39 \\
\hline 26 & 15 & 18.50 & 17.21 & 54 & 36 & 51.50 & 33.84 \\
\hline 17 & 26 & 21.50 & 29.14 & 44 & 54 & 50.50 & 58.23 \\
\hline 21 & 17 & 21.50 & 14.64 & 49 & 44 & 50.00 & 38.78 \\
\hline 26 & 21 & 18.50 & 23.84 & 68 & 49 & 79.00 & 50.70 \\
\hline 24 & 26 & 25.50 & 27.13 & 84 & 68 & 83.00 & 70.16 \\
\hline 20 & 24 & 22.50 & 23.72 & 134 & 84 & 123.08 & 83.72 \\
\hline 30 & 20 & 31.50 & 19.89 & 126 & 134 & 123.15 & 140.28 \\
\hline 16 & 30 & 20.83 & 33.16 & 106 & 126 & 111.42 & 113.61 \\
\hline 11 & 16 & 7.50 & 12.19 & 103 & 106 & 112.50 & 97.42 \\
\hline 10 & 11 & 12.50 & 12.43 & 98 & 103 & 107.50 & 98.68 \\
\hline 5 & 10 & 10.50 & 11.14 & 53 & 98 & 50.50 & 92.27 \\
\hline 2 & 5 & 5.50 & 5.45 & 61 & 53 & 62.50 & 39.66 \\
\hline 9 & 2 & 9.50 & 3.51 & 84 & 61 & 83.00 & 65.19 \\
\hline 9 & 9 & 9.50 & 12.57 & 63 & 84 & 53.50 & 85.41 \\
\hline 2 & 9 & 5.50 & 9.84 & 36 & 63 & 34.83 & 53.88 \\
\hline 8 & 2 & 9.50 & 2.17 & 31 & 36 & 36.50 & 30.64 \\
\hline 6 & 8 & 5.50 & 11.74 & 48 & 31 & 42.50 & 31.57 \\
\hline 5 & 6 & 10.50 & 6.42 & 60 & 48 & 51.00 & 51.90 \\
\hline 2 & 5 & 5.50 & 6.80 & 71 & 60 & 73.25 & 60.34 \\
\hline 2 & 2 & 5.50 & 3.04 & 49 & 71 & 50.00 & 71.14 \\
\hline 2 & 2 & 5.50 & 4.16 & 59 & 49 & 62.00 & 41.23 \\
\hline 5 & 2 & 10.50 & 3.81 & 83 & 59 & 52.50 & 62.36 \\
\hline 6 & 5 & 5.50 & 7.54 & 88 & 83 & 86.83 & 85.11 \\
\hline
\end{tabular}




\begin{tabular}{|c|c|c|c|c|c|c|c|}
\hline 85 & 88 & 70.50 & 84.35 & 19 & 8 & 25.50 & 11.53 \\
\hline 80 & 85 & 67.83 & 80.96 & 16 & 19 & 20.83 & 22.74 \\
\hline 86 & 80 & 62.50 & 75.94 & 11 & 16 & 7.50 & 15.73 \\
\hline 85 & 86 & 70.50 & 84.74 & 10 & 11 & 12.50 & 11.77 \\
\hline 78 & 85 & 85.50 & 80.90 & 8 & 10 & 9.50 & 11.73 \\
\hline 81 & 78 & 83.03 & 73.59 & 12 & 8 & 9.50 & 9.31 \\
\hline 65 & 81 & 66.67 & 79.44 & 14 & 12 & 20.50 & 14.88 \\
\hline 57 & 65 & 54.50 & 58.30 & 17 & 14 & 21.50 & 15.62 \\
\hline 34 & 57 & 26.50 & 54.97 & 24 & 17 & 25.50 & 19.02 \\
\hline 19 & 34 & 25.50 & 28.09 & 24 & 24 & 25.50 & 26.48 \\
\hline 34 & 19 & 26.50 & 17.98 & 37 & 24 & 39.50 & 24.23 \\
\hline 25 & 34 & 28.50 & 39.20 & 40 & 37 & 37.50 & 40.66 \\
\hline 30 & 25 & 31.50 & 21.91 & 38 & 40 & 31.50 & 39.36 \\
\hline 37 & 30 & 39.50 & 33.16 & 51 & 38 & 37.50 & 37.33 \\
\hline 33 & 37 & 32.50 & 38.26 & 80 & 51 & 67.83 & 53.70 \\
\hline 37 & 33 & 39.50 & 31.88 & 101 & 80 & 88.50 & 83.95 \\
\hline 47 & 37 & 59.50 & 38.64 & 98 & 101 & 107.50 & 100.33 \\
\hline 33 & 47 & 32.50 & 48.73 & 116 & 98 & 109.50 & 91.79 \\
\hline 35 & 33 & 36.00 & 28.73 & 135 & 116 & 133.00 & 116.21 \\
\hline 30 & 35 & 31.50 & 37.17 & 108 & 135 & 105.17 & 131.94 \\
\hline 37 & 30 & 39.50 & 28.56 & 89 & 108 & 88.50 & 94.51 \\
\hline 27 & 37 & 29.50 & 39.63 & 89 & 89 & 88.50 & 82.76 \\
\hline 48 & 27 & 42.50 & 24.18 & 88 & 89 & 86.83 & 86.31 \\
\hline 42 & 48 & 41.50 & 54.28 & 56 & 88 & 53.50 & 84.05 \\
\hline 29 & 42 & 38.50 & 37.96 & 103 & 56 & 112.50 & 45.95 \\
\hline 32 & 29 & 35.30 & 27.11 & 72 & 103 & 67.50 & 114.40 \\
\hline 27 & 32 & 29.50 & 34.00 & 65 & 72 & 66.67 & 56.26 \\
\hline 37 & 27 & 39.50 & 25.87 & 59 & 65 & 62.00 & 65.27 \\
\hline 43 & 37 & 44.00 & 40.43 & 49 & 59 & 50.00 & 55.30 \\
\hline 43 & 43 & 44.00 & 43.33 & 45 & 49 & 45.83 & 46.18 \\
\hline 45 & 43 & 45.83 & 42.46 & 34 & 45 & 26.50 & 44.07 \\
\hline 39 & 45 & 37.10 & 45.15 & 49 & 34 & 50.00 & 31.37 \\
\hline 31 & 39 & 36.50 & 37.07 & 52 & 49 & 51.50 & 53.38 \\
\hline 28 & 31 & 33.50 & 29.80 & 41 & 52 & 38.10 & 50.40 \\
\hline 22 & 28 & 21.50 & 28.34 & 39 & 41 & 37.10 & 37.96 \\
\hline 19 & 22 & 25.50 & 21.50 & 31 & 39 & 36.50 & 39.28 \\
\hline 22 & 19 & 21.50 & 19.91 & 18 & 31 & 15.83 & 29.18 \\
\hline 15 & 22 & 13.50 & 24.02 & 17 & 18 & 21.50 & 16.45 \\
\hline 12 & 15 & 9.50 & 14.29 & 14 & 17 & 20.50 & 19.06 \\
\hline 6 & 12 & 5.50 & 13.57 & 10 & 14 & 12.50 & 14.63 \\
\hline 3 & 6 & 4.50 & 6.50 & 11 & 10 & 7.50 & 11.10 \\
\hline 1 & 3 & 0.00 & 4.97 & 22 & 11 & 21.50 & 13.36 \\
\hline 0 & 1 & 0.00 & 2.99 & 19 & 22 & 25.50 & 26.01 \\
\hline 0 & 0 & 0.00 & 2.36 & 87 & 19 & 80.50 & 18.56 \\
\hline 0 & 0 & 0.00 & 2.54 & 108 & 87 & 105.17 & 103.24 \\
\hline 1 & 0 & 0.00 & 2.47 & 105 & 108 & 99.59 & 103.26 \\
\hline 2 & 1 & 5.50 & 3.69 & 63 & 105 & 53.50 & 99.64 \\
\hline 8 & 2 & 9.50 & 4.52 & 45 & 63 & 45.83 & 49.83 \\
\hline
\end{tabular}




\begin{tabular}{|c|c|c|c|c|c|c|c|}
\hline 21 & 45 & 21.50 & 43.00 & 88 & 71 & 86.83 & 78.94 \\
\hline 46 & 21 & 47.00 & 15.96 & 89 & 88 & 88.50 & 86.30 \\
\hline 46 & 46 & 47.00 & 54.39 & 80 & 89 & 67.83 & 85.32 \\
\hline 43 & 46 & 44.00 & 42.84 & 103 & 80 & 112.50 & 74.72 \\
\hline 22 & 43 & 21.50 & 42.68 & 105 & 103 & 99.59 & 105.81 \\
\hline 11 & 22 & 7.50 & 17.26 & 97 & 105 & 109.50 & 98.91 \\
\hline 12 & 11 & 9.50 & 11.56 & 101 & 97 & 88.50 & 91.32 \\
\hline 9 & 12 & 9.50 & 14.48 & 111 & 101 & 117.50 & 98.47 \\
\hline 2 & 9 & 5.50 & 9.95 & 100 & 111 & 94.50 & 108.47 \\
\hline 2 & 2 & 5.50 & 2.81 & 94 & 100 & 91.57 & 92.16 \\
\hline 5 & 2 & 10.50 & 4.94 & 99 & 94 & 93.28 & 89.81 \\
\hline 7 & 5 & 10.50 & 7.92 & 112 & 99 & 127.50 & 96.60 \\
\hline 1 & 7 & 0.00 & 9.44 & 123 & 112 & 113.50 & 110.34 \\
\hline 3 & 1 & 4.50 & 1.70 & 131 & 123 & 134.50 & 119.58 \\
\hline 6 & 3 & 5.50 & 6.43 & 132 & 131 & 132.50 & 126.53 \\
\hline 9 & 6 & 9.50 & 8.63 & 142 & 132 & 141.25 & 125.69 \\
\hline 11 & 9 & 7.50 & 11.60 & 99 & 142 & 93.28 & 138.11 \\
\hline 16 & 11 & 20.83 & 13.12 & 102 & 99 & 94.00 & 82.28 \\
\hline 22 & 16 & 21.50 & 18.71 & 112 & 102 & 127.50 & 102.72 \\
\hline 19 & 22 & 25.50 & 24.29 & 109 & 112 & 110.69 & 108.72 \\
\hline 22 & 19 & 21.50 & 18.97 & 92 & 109 & 73.83 & 103.31 \\
\hline 36 & 22 & 34.83 & 24.20 & 79 & 92 & 68.50 & 84.35 \\
\hline 37 & 36 & 39.50 & 39.60 & 50 & 79 & 45.50 & 74.31 \\
\hline 54 & 37 & 51.50 & 36.18 & 30 & 50 & 31.50 & 42.18 \\
\hline 38 & 54 & 31.50 & 57.82 & 20 & 30 & 22.50 & 27.60 \\
\hline 32 & 38 & 35.30 & 31.92 & 27 & 20 & 29.50 & 19.85 \\
\hline 28 & 32 & 33.50 & 32.43 & 70 & 27 & 77.50 & 30.66 \\
\hline 10 & 28 & 12.50 & 27.43 & 102 & 70 & 94.00 & 79.54 \\
\hline 8 & 10 & 9.50 & 7.10 & 56 & 102 & 53.50 & 103.65 \\
\hline 9 & 8 & 9.50 & 10.78 & 62 & 56 & 78.00 & 40.65 \\
\hline 6 & 9 & 5.50 & 10.87 & 87 & 62 & 80.50 & 66.87 \\
\hline 4 & 6 & 6.50 & 7.20 & 81 & 87 & 83.03 & 89.30 \\
\hline 14 & 4 & 20.50 & 5.86 & 61 & 81 & 62.50 & 75.30 \\
\hline 16 & 14 & 20.83 & 18.38 & 35 & 61 & 36.00 & 55.28 \\
\hline 19 & 16 & 25.50 & 17.03 & 23 & 35 & 22.50 & 29.78 \\
\hline 33 & 19 & 32.50 & 21.06 & 20 & 23 & 22.50 & 22.89 \\
\hline 69 & 33 & 63.50 & 36.82 & 23 & 20 & 22.50 & 21.32 \\
\hline 91 & 69 & 65.50 & 75.73 & 7 & 23 & 10.50 & 25.42 \\
\hline 83 & 91 & 52.50 & 90.71 & 6 & 7 & 5.50 & 4.77 \\
\hline 54 & 83 & 51.50 & 76.53 & 31 & 6 & 36.50 & 9.75 \\
\hline 44 & 54 & 50.50 & 45.65 & 15 & 31 & 13.50 & 38.55 \\
\hline 66 & 44 & 79.50 & 42.82 & 18 & 15 & 15.83 & 10.49 \\
\hline 113 & 66 & 103.21 & 70.35 & 38 & 18 & 31.50 & 22.55 \\
\hline 136 & 113 & 116.50 & 119.07 & 31 & 38 & 36.50 & 43.16 \\
\hline 129 & 136 & 121.28 & 132.34 & 35 & 31 & 36.00 & 28.47 \\
\hline 57 & 129 & 54.50 & 119.90 & 28 & 35 & 33.50 & 37.73 \\
\hline 35 & 57 & 36.00 & 36.38 & 27 & 28 & 29.50 & 26.46 \\
\hline 71 & 35 & 73.25 & 34.83 & 18 & 27 & 15.83 & 28.63 \\
\hline
\end{tabular}




\begin{tabular}{|c|c|c|c|c|c|c|c|}
\hline 23 & 18 & 22.50 & 17.06 & 8 & 11 & 9.50 & 12.09 \\
\hline 40 & 23 & 37.50 & 26.59 & 4 & 8 & 6.50 & 9.97 \\
\hline 45 & 40 & 45.83 & 44.33 & 13 & 4 & 12.50 & 5.74 \\
\hline 95 & 45 & 104.50 & 45.05 & 14 & 13 & 20.50 & 17.91 \\
\hline 89 & 95 & 88.50 & 105.46 & 9 & 14 & 9.50 & 15.45 \\
\hline 101 & 89 & 88.50 & 80.04 & 8 & 9 & 9.50 & 10.11 \\
\hline 115 & 101 & 111.50 & 102.25 & 6 & 8 & 5.50 & 10.49 \\
\hline 103 & 115 & 112.50 & 112.57 & 12 & 6 & 9.50 & 7.94 \\
\hline 128 & 103 & 112.50 & 94.94 & 19 & 12 & 25.50 & 15.96 \\
\hline 136 & 128 & 116.50 & 130.58 & 24 & 19 & 25.50 & 22.02 \\
\hline 95 & 136 & 104.50 & 129.60 & 28 & 24 & 33.50 & 26.25 \\
\hline 74 & 95 & 77.50 & 80.22 & 29 & 28 & 38.50 & 29.82 \\
\hline 61 & 74 & 62.50 & 69.62 & 34 & 29 & 26.50 & 29.96 \\
\hline 51 & 61 & 37.50 & 57.06 & 34 & 34 & 26.50 & 35.97 \\
\hline 37 & 51 & 39.50 & 48.72 & 39 & 34 & 37.10 & 34.16 \\
\hline 43 & 37 & 44.00 & 34.25 & 66 & 39 & 79.50 & 40.76 \\
\hline 68 & 43 & 79.00 & 45.87 & 111 & 66 & 117.50 & 71.51 \\
\hline 62 & 68 & 78.00 & 72.69 & 97 & 111 & 109.50 & 116.84 \\
\hline 64 & 62 & 62.21 & 57.36 & 47 & 97 & 59.50 & 86.26 \\
\hline 61 & 64 & 62.50 & 64.40 & 73 & 47 & 79.50 & 34.85 \\
\hline 53 & 61 & 50.50 & 58.65 & 73 & 73 & 79.50 & 81.84 \\
\hline 55 & 53 & 72.50 & 50.69 & 71 & 73 & 73.25 & 67.72 \\
\hline 57 & 55 & 54.50 & 55.51 & 71 & 71 & 73.25 & 69.55 \\
\hline 45 & 57 & 45.83 & 56.49 & 71 & 71 & 73.25 & 69.01 \\
\hline 45 & 45 & 45.83 & 41.65 & 57 & 71 & 54.50 & 69.18 \\
\hline 45 & 45 & 45.83 & 46.11 & 35 & 57 & 36.00 & 52.17 \\
\hline 45 & 45 & 45.83 & 44.77 & 51 & 35 & 37.50 & 30.62 \\
\hline 45 & 45 & 45.83 & 45.17 & 35 & 51 & 36.00 & 56.49 \\
\hline 45 & 45 & 45.83 & 45.05 & 56 & 35 & 53.50 & 29.31 \\
\hline 45 & 45 & 45.83 & 45.08 & 69 & 56 & 63.50 & 62.94 \\
\hline 59 & 45 & 62.00 & 45.07 & 57 & 69 & 54.50 & 68.60 \\
\hline 72 & 59 & 67.50 & 62.05 & 33 & 57 & 32.50 & 52.36 \\
\hline 64 & 72 & 62.21 & 72.71 & 37 & 33 & 39.50 & 28.15 \\
\hline 53 & 64 & 50.50 & 59.81 & 34 & 37 & 26.50 & 40.27 \\
\hline 40 & 53 & 37.50 & 50.36 & 39 & 34 & 37.10 & 32.99 \\
\hline 27 & 40 & 29.50 & 37.44 & 48 & 39 & 42.50 & 41.23 \\
\hline 21 & 27 & 21.50 & 25.56 & 53 & 48 & 50.50 & 49.66 \\
\hline 24 & 21 & 25.50 & 21.85 & 35 & 53 & 36.00 & 53.19 \\
\hline 13 & 24 & 12.50 & 26.60 & 26 & 35 & 18.50 & 30.31 \\
\hline 3 & 13 & 4.50 & 11.82 & 31 & 26 & 36.50 & 26.28 \\
\hline 3 & 3 & 4.50 & 4.13 & 23 & 31 & 22.50 & 33.54 \\
\hline 5 & 3 & 10.50 & 6.42 & 15 & 23 & 13.50 & 21.65 \\
\hline 5 & 5 & 10.50 & 8.14 & 9 & 15 & 9.50 & 15.52 \\
\hline 5 & 5 & 10.50 & 7.61 & 12 & 9 & 9.50 & 10.08 \\
\hline 10 & 5 & 12.50 & 7.75 & 48 & 12 & 42.50 & 15.34 \\
\hline 17 & 10 & 21.50 & 13.75 & 49 & 48 & 50.00 & 57.39 \\
\hline 16 & 17 & 20.83 & 20.42 & 55 & 49 & 72.50 & 45.96 \\
\hline 11 & 16 & 7.50 & 17.19 & 33 & 55 & 32.50 & 56.67 \\
\hline
\end{tabular}




\begin{tabular}{|c|c|c|c|c|c|c|c|}
\hline 28 & 33 & 33.50 & 26.78 & 41 & 56 & 38.10 & 54.71 \\
\hline 44 & 28 & 50.50 & 29.70 & 13 & 41 & 12.50 & 36.75 \\
\hline 46 & 44 & 47.00 & 48.22 & 9 & 13 & 9.50 & 8.20 \\
\hline 38 & 46 & 31.50 & 45.07 & 11 & 9 & 7.50 & 11.93 \\
\hline 28 & 38 & 33.50 & 36.32 & 6 & 11 & 5.50 & 13.22 \\
\hline 24 & 28 & 25.50 & 26.83 & 10 & 6 & 12.50 & 6.76 \\
\hline 31 & 24 & 36.50 & 24.82 & 11 & 10 & 7.50 & 13.53 \\
\hline 29 & 31 & 38.50 & 33.90 & 16 & 11 & 20.83 & 12.70 \\
\hline 21 & 29 & 21.50 & 28.74 & 17 & 16 & 21.50 & 19.00 \\
\hline 28 & 21 & 33.50 & 20.59 & 19 & 17 & 25.50 & 18.31 \\
\hline 25 & 28 & 28.50 & 31.52 & 15 & 19 & 13.50 & 20.93 \\
\hline 16 & 25 & 20.83 & 24.59 & 18 & 15 & 15.83 & 15.28 \\
\hline 12 & 16 & 9.50 & 15.75 & 37 & 18 & 39.50 & 20.61 \\
\hline 10 & 12 & 12.50 & 13.55 & 57 & 37 & 54.50 & 42.04 \\
\hline 16 & 10 & 20.83 & 11.77 & 53 & 57 & 50.50 & 59.84 \\
\hline 19 & 16 & 25.50 & 19.57 & 50 & 53 & 45.50 & 49.64 \\
\hline 16 & 19 & 20.83 & 20.85 & 60 & 50 & 51.00 & 49.08 \\
\hline 18 & 16 & 15.83 & 16.82 & 68 & 60 & 79.00 & 61.38 \\
\hline 25 & 18 & 28.50 & 20.45 & 58 & 68 & 57.50 & 67.39 \\
\hline 40 & 25 & 37.50 & 27.83 & 74 & 58 & 77.50 & 53.47 \\
\hline 39 & 40 & 37.10 & 43.79 & 53 & 74 & 50.50 & 77.06 \\
\hline 33 & 39 & 32.50 & 37.78 & 46 & 53 & 47.00 & 44.52 \\
\hline 38 & 33 & 31.50 & 32.31 & 40 & 46 & 37.50 & 45.82 \\
\hline 27 & 38 & 29.50 & 40.01 & 37 & 40 & 39.50 & 38.16 \\
\hline 30 & 27 & 31.50 & 24.36 & 13 & 37 & 12.50 & 36.82 \\
\hline 13 & 30 & 12.50 & 32.70 & 12 & 13 & 9.50 & 8.12 \\
\hline 10 & 13 & 12.50 & 9.57 & 20 & 12 & 22.50 & 15.53 \\
\hline 6 & 10 & 5.50 & 12.88 & 25 & 20 & 28.50 & 22.99 \\
\hline 8 & 6 & 9.50 & 7.02 & 27 & 25 & 29.50 & 26.80 \\
\hline 6 & 8 & 5.50 & 11.19 & 18 & 27 & 15.83 & 28.08 \\
\hline 3 & 6 & 4.50 & 7.50 & 15 & 18 & 13.50 & 16.78 \\
\hline 3 & 3 & 4.50 & 4.96 & 13 & 15 & 12.50 & 16.53 \\
\hline 6 & 3 & 5.50 & 5.70 & 24 & 13 & 25.50 & 14.17 \\
\hline 6 & 6 & 5.50 & 9.10 & 24 & 24 & 25.50 & 28.20 \\
\hline 10 & 6 & 12.50 & 8.06 & 21 & 24 & 21.50 & 23.98 \\
\hline 21 & 10 & 21.50 & 13.21 & 25 & 21 & 28.50 & 21.60 \\
\hline 26 & 21 & 18.50 & 24.99 & 32 & 25 & 35.30 & 27.16 \\
\hline 25 & 26 & 28.50 & 27.50 & 37 & 32 & 39.50 & 33.97 \\
\hline 25 & 25 & 28.50 & 25.53 & 32 & 37 & 35.30 & 37.98 \\
\hline 27 & 25 & 29.50 & 26.11 & 43 & 32 & 44.00 & 30.71 \\
\hline 32 & 27 & 35.30 & 28.36 & 56 & 43 & 53.50 & 46.23 \\
\hline 38 & 32 & 31.50 & 33.74 & 53 & 56 & 50.50 & 57.33 \\
\hline 47 & 38 & 59.50 & 39.39 & 39 & 53 & 37.10 & 50.36 \\
\hline 47 & 47 & 59.50 & 48.60 & 39 & 39 & 37.10 & 35.49 \\
\hline 48 & 47 & 42.50 & 45.84 & 49 & 39 & 50.00 & 39.96 \\
\hline 56 & 48 & 53.50 & 47.88 & 52 & 49 & 51.50 & 50.74 \\
\hline 57 & 56 & 54.50 & 56.97 & 52 & 52 & 51.50 & 51.14 \\
\hline 56 & 57 & 53.50 & 55.46 & 59 & 52 & 62.00 & 51.03 \\
\hline
\end{tabular}




\begin{tabular}{|c|c|c|c|c|c|c|c|}
\hline 80 & 59 & 67.83 & 59.55 & 39 & 31 & 37.10 & 35.31 \\
\hline 90 & 80 & 72.50 & 82.46 & 61 & 39 & 62.50 & 40.43 \\
\hline 94 & 90 & 91.57 & 87.71 & 57 & 61 & 54.50 & 65.56 \\
\hline 89 & 94 & 88.50 & 91.00 & 57 & 57 & 54.50 & 53.16 \\
\hline 128 & 89 & 112.50 & 83.97 & 58 & 57 & 57.50 & 56.89 \\
\hline 112 & 128 & 127.50 & 133.39 & 48 & 58 & 42.50 & 56.99 \\
\hline 111 & 112 & 117.50 & 99.17 & 43 & 48 & 44.00 & 44.84 \\
\hline 95 & 111 & 104.50 & 108.28 & 41 & 43 & 38.10 & 42.43 \\
\hline 111 & 95 & 117.50 & 86.17 & 38 & 41 & 31.50 & 40.73 \\
\hline 95 & 111 & 104.50 & 112.24 & 39 & 38 & 37.10 & 37.61 \\
\hline 69 & 95 & 63.50 & 85.03 & 25 & 39 & 28.50 & 39.76 \\
\hline 47 & 69 & 59.50 & 61.71 & 11 & 25 & 7.50 & 22.13 \\
\hline 73 & 47 & 79.50 & 42.06 & 9 & 11 & 9.50 & 10.45 \\
\hline 69 & 73 & 63.50 & 79.49 & 9 & 9 & 9.50 & 11.52 \\
\hline 48 & 69 & 42.50 & 63.40 & 8 & 9 & 9.50 & 11.19 \\
\hline 35 & 48 & 36.00 & 42.79 & 9 & 8 & 9.50 & 10.06 \\
\hline 20 & 35 & 22.50 & 33.22 & 8 & 9 & 9.50 & 11.60 \\
\hline 10 & 20 & 12.50 & 17.91 & 2 & 8 & 5.50 & 9.91 \\
\hline 22 & 10 & 21.50 & 10.38 & 0 & 2 & 0.00 & 3.13 \\
\hline 6 & 22 & 5.50 & 27.18 & 0 & 0 & 0.00 & 2.73 \\
\hline 8 & 6 & 9.50 & 2.72 & 0 & 0 & 0.00 & 2.83 \\
\hline 25 & 8 & 28.50 & 12.49 & 0 & 0 & 0.00 & 2.78 \\
\hline 33 & 25 & 32.50 & 30.15 & 0 & 0 & 0.00 & 2.78 \\
\hline 32 & 33 & 35.30 & 34.53 & 0 & 0 & 0.00 & 2.76 \\
\hline 16 & 32 & 20.83 & 32.00 & 1 & 0 & 0.00 & 2.75 \\
\hline 16 & 16 & 20.83 & 13.35 & 6 & 1 & 5.50 & 3.95 \\
\hline 14 & 16 & 20.50 & 18.95 & 14 & 6 & 20.50 & 9.63 \\
\hline 26 & 14 & 18.50 & 14.83 & 9 & 14 & 9.50 & 17.61 \\
\hline 26 & 26 & 18.50 & 30.61 & 6 & 9 & 5.50 & 9.14 \\
\hline 35 & 26 & 36.00 & 25.86 & 4 & 6 & 6.50 & 8.04 \\
\hline 46 & 35 & 47.00 & 38.19 & 4 & 4 & 6.50 & 5.93 \\
\hline 98 & 46 & 107.50 & 47.82 & 4 & 4 & 6.50 & 6.55 \\
\hline 134 & 98 & 123.08 & 107.97 & 2 & 4 & 5.50 & 6.35 \\
\hline 137 & 134 & 128.36 & 133.56 & 1 & 2 & 0.00 & 3.97 \\
\hline 128 & 137 & 112.50 & 129.54 & 3 & 1 & 4.50 & 3.45 \\
\hline 109 & 128 & 110.69 & 119.87 & 11 & 3 & 7.50 & 6.02 \\
\hline 102 & 109 & 94.00 & 99.78 & 11 & 11 & 7.50 & 14.93 \\
\hline 95 & 102 & 104.50 & 97.36 & 20 & 11 & 22.50 & 12.24 \\
\hline 115 & 95 & 111.50 & 89.63 & 21 & 20 & 21.50 & 23.95 \\
\hline 102 & 115 & 94.00 & 116.22 & 18 & 21 & 15.83 & 21.63 \\
\hline 65 & 102 & 66.67 & 92.49 & 14 & 18 & 20.50 & 18.68 \\
\hline 40 & 65 & 37.50 & 54.79 & 13 & 14 & 12.50 & 14.71 \\
\hline 25 & 40 & 28.50 & 35.82 & 3 & 13 & 4.50 & 14.68 \\
\hline 23 & 25 & 22.50 & 23.34 & 0 & 3 & 0.00 & 2.56 \\
\hline 32 & 23 & 35.30 & 24.66 & 2 & 0 & 5.50 & 2.55 \\
\hline 34 & 32 & 26.50 & 35.17 & 3 & 2 & 4.50 & 4.96 \\
\hline 22 & 34 & 21.50 & 34.43 & 3 & 3 & 4.50 & 5.43 \\
\hline 31 & 22 & 36.50 & 20.10 & 0 & 3 & 0.00 & 5.28 \\
\hline
\end{tabular}




\begin{tabular}{|c|c|c|c|c|c|c|c|}
\hline 0 & 0 & 0.00 & 1.67 & 1 & 4 & 0.00 & 6.25 \\
\hline 0 & 0 & 0.00 & 2.74 & 0 & 1 & 0.00 & 2.03 \\
\hline 0 & 0 & 0.00 & 2.41 & 1 & 0 & 0.00 & 2.07 \\
\hline 0 & 0 & 0.00 & 2.49 & 2 & 1 & 5.50 & 3.26 \\
\hline 0 & 0 & 0.00 & 2.45 & 2 & 2 & 5.50 & 4.10 \\
\hline 0 & 0 & 0.00 & 2.45 & 1 & 2 & 0.00 & 3.84 \\
\hline 0 & 0 & 0.00 & 2.43 & 1 & 1 & 0.00 & 2.69 \\
\hline 0 & 0 & 0.00 & 2.42 & 0 & 1 & 0.00 & 3.02 \\
\hline 0 & 0 & 0.00 & 2.41 & 0 & 0 & 0.00 & 1.70 \\
\hline 1 & 0 & 0.00 & 2.40 & 0 & 0 & 0.00 & 2.09 \\
\hline 2 & 1 & 5.50 & 3.60 & 3 & 0 & 4.50 & 1.96 \\
\hline 6 & 2 & 5.50 & 4.44 & 4 & 3 & 6.50 & 5.62 \\
\hline 8 & 6 & 9.50 & 9.02 & 4 & 4 & 6.50 & 5.72 \\
\hline 4 & 8 & 6.50 & 10.06 & 1 & 4 & 0.00 & 5.68 \\
\hline 2 & 4 & 5.50 & 4.88 & 0 & 1 & 0.00 & 2.04 \\
\hline 3 & 2 & 4.50 & 4.00 & 2 & 0 & 5.50 & 1.91 \\
\hline 12 & 3 & 9.50 & 5.46 & 14 & 2 & 20.50 & 4.37 \\
\hline 10 & 12 & 12.50 & 15.92 & 17 & 14 & 21.50 & 18.17 \\
\hline 16 & 10 & 20.83 & 10.34 & 15 & 17 & 13.50 & 17.65 \\
\hline 7 & 16 & 10.50 & 19.29 & 13 & 15 & 12.50 & 15.37 \\
\hline 9 & 7 & 9.50 & 5.68 & 8 & 13 & 9.50 & 13.63 \\
\hline 15 & 9 & 13.50 & 12.18 & 5 & 8 & 10.50 & 8.08 \\
\hline 10 & 15 & 12.50 & 17.49 & 7 & 5 & 10.50 & 6.10 \\
\hline 9 & 10 & 9.50 & 9.82 & 13 & 7 & 12.50 & 9.11 \\
\hline 5 & 9 & 10.50 & 10.91 & 8 & 13 & 9.50 & 15.48 \\
\hline 0 & 5 & 0.00 & 5.72 & 1 & 8 & 0.00 & 7.49 \\
\hline 0 & 0 & 0.00 & 1.21 & 0 & 1 & 0.00 & 1.40 \\
\hline 0 & 0 & 0.00 & 2.55 & 0 & 0 & 0.00 & 2.01 \\
\hline 1 & 0 & 0.00 & 2.13 & 0 & 0 & 0.00 & 1.81 \\
\hline 2 & 1 & 5.50 & 3.46 & 1 & 0 & 0.00 & 1.86 \\
\hline 2 & 2 & 5.50 & 4.26 & 1 & 1 & 0.00 & 3.05 \\
\hline 0 & 2 & 0.00 & 4.00 & 0 & 1 & 0.00 & 2.68 \\
\hline 0 & 0 & 0.00 & 1.64 & 0 & 0 & 0.00 & 1.57 \\
\hline 2 & 0 & 5.50 & 2.34 & 2 & 0 & 5.50 & 1.89 \\
\hline 4 & 2 & 6.50 & 4.54 & 3 & 2 & 4.50 & 4.21 \\
\hline 5 & 4 & 10.50 & 6.29 & 16 & 3 & 20.83 & 4.71 \\
\hline 10 & 5 & 12.50 & 6.97 & 17 & 16 & 21.50 & 20.31 \\
\hline 12 & 10 & 9.50 & 12.81 & 18 & 17 & 15.83 & 16.83 \\
\hline 9 & 12 & 9.50 & 13.47 & 37 & 18 & 39.50 & 19.08 \\
\hline 15 & 9 & 13.50 & 9.63 & 25 & 37 & 28.50 & 41.44 \\
\hline 21 & 15 & 21.50 & 18.05 & 26 & 25 & 18.50 & 20.17 \\
\hline 28 & 21 & 33.50 & 22.78 & 34 & 26 & 26.50 & 27.78 \\
\hline 25 & 28 & 28.50 & 29.84 & 45 & 34 & 45.83 & 35.19 \\
\hline 18 & 25 & 15.83 & 24.08 & 55 & 45 & 72.50 & 46.30 \\
\hline 11 & 18 & 7.50 & 17.32 & 53 & 55 & 50.50 & 55.09 \\
\hline 6 & 11 & 5.50 & 10.86 & 53 & 53 & 50.50 & 50.04 \\
\hline 3 & 6 & 4.50 & 6.74 & 51 & 53 & 37.50 & 51.57 \\
\hline 4 & 3 & 6.50 & 4.33 & 37 & 51 & 39.50 & 48.69 \\
\hline
\end{tabular}




\begin{tabular}{|c|c|c|c|c|c|c|c|}
\hline 18 & 37 & 15.83 & 32.59 & 9 & 8 & 9.50 & 9.70 \\
\hline 11 & 18 & 7.50 & 14.40 & 8 & 9 & 9.50 & 10.55 \\
\hline 11 & 11 & 7.50 & 11.38 & 7 & 8 & 10.50 & 9.07 \\
\hline 10 & 11 & 12.50 & 12.28 & 7 & 7 & 10.50 & 8.30 \\
\hline 10 & 10 & 12.50 & 10.79 & 1 & 7 & 0.00 & 8.52 \\
\hline 12 & 10 & 9.50 & 11.23 & 5 & 1 & 10.50 & 1.17 \\
\hline 15 & 12 & 13.50 & 13.52 & 14 & 5 & 20.50 & 8.22 \\
\hline 17 & 15 & 21.50 & 16.46 & 33 & 14 & 32.50 & 17.00 \\
\hline 27 & 17 & 29.50 & 17.99 & 35 & 33 & 36.00 & 37.39 \\
\hline 36 & 27 & 34.83 & 29.65 & 23 & 35 & 22.50 & 33.68 \\
\hline 32 & 36 & 35.30 & 37.06 & 18 & 23 & 15.83 & 20.25 \\
\hline 54 & 32 & 51.50 & 29.99 & 19 & 18 & 25.50 & 18.23 \\
\hline 62 & 54 & 78.00 & 58.79 & 2 & 19 & 5.50 & 20.04 \\
\hline 61 & 62 & 62.50 & 59.84 & 0 & 2 & 0.00 & -1.12 \\
\hline 71 & 61 & 73.25 & 58.33 & 0 & 0 & 0.00 & 2.81 \\
\hline 66 & 71 & 79.50 & 70.92 & 0 & 0 & 0.00 & 1.62 \\
\hline 86 & 66 & 62.50 & 61.09 & 3 & 0 & 4.50 & 1.96 \\
\hline 119 & 86 & 126.50 & 88.31 & 6 & 3 & 5.50 & 5.48 \\
\hline 106 & 119 & 111.42 & 120.16 & 22 & 6 & 21.50 & 8.05 \\
\hline 99 & 106 & 93.28 & 94.86 & 35 & 22 & 36.00 & 26.67 \\
\hline 89 & 99 & 88.50 & 94.01 & 46 & 35 & 47.00 & 36.83 \\
\hline 84 & 89 & 83.00 & 82.17 & 48 & 46 & 42.50 & 47.12 \\
\hline 84 & 84 & 83.00 & 79.69 & 47 & 48 & 59.50 & 46.46 \\
\hline 87 & 84 & 80.50 & 80.46 & 36 & 47 & 34.83 & 45.45 \\
\hline 65 & 87 & 66.67 & 83.89 & 24 & 36 & 25.50 & 32.42 \\
\hline 39 & 65 & 37.10 & 56.20 & 13 & 24 & 12.50 & 21.79 \\
\hline 36 & 39 & 34.83 & 33.02 & 9 & 13 & 9.50 & 11.65 \\
\hline 27 & 36 & 29.50 & 36.36 & 19 & 9 & 25.50 & 9.84 \\
\hline 23 & 27 & 22.50 & 24.44 & 39 & 19 & 37.10 & 22.50 \\
\hline 33 & 23 & 32.50 & 23.17 & 56 & 39 & 53.50 & 42.94 \\
\hline 39 & 33 & 37.10 & 35.68 & 73 & 56 & 79.50 & 57.41 \\
\hline 30 & 39 & 31.50 & 39.19 & 105 & 73 & 99.59 & 73.69 \\
\hline 24 & 30 & 25.50 & 27.23 & 116 & 105 & 109.50 & 107.61 \\
\hline 23 & 24 & 22.50 & 23.55 & 113 & 116 & 103.21 & 110.78 \\
\hline 12 & 23 & 9.50 & 23.44 & 100 & 113 & 94.50 & 106.22 \\
\hline 10 & 12 & 12.50 & 10.13 & 105 & 100 & 99.59 & 91.86 \\
\hline 10 & 10 & 12.50 & 11.70 & 156 & 105 & 158.00 & 102.27 \\
\hline 21 & 10 & 21.50 & 11.22 & 156 & 156 & 158.00 & 161.01 \\
\hline 27 & 21 & 29.50 & 24.70 & 160 & 156 & 161.50 & 143.40 \\
\hline 25 & 27 & 28.50 & 27.91 & 165 & 160 & 170.50 & 153.60 \\
\hline 35 & 25 & 36.00 & 24.52 & 159 & 165 & 160.50 & 156.64 \\
\hline 27 & 35 & 29.50 & 37.66 & & & & \\
\hline 18 & 27 & 15.83 & 24.01 & & & & \\
\hline 15 & 18 & 13.50 & 17.20 & & & & \\
\hline 9 & 15 & 9.50 & 15.61 & & & & \\
\hline 7 & 9 & 10.50 & 8.81 & & & & \\
\hline 7 & 7 & 10.50 & 8.42 & & & & \\
\hline 8 & 7 & 9.50 & 8.53 & & & & \\
\hline
\end{tabular}




\subsubsection{ERCOT System-wide Forecasted Data}

The data below gives the forecasts produced by each model over the period of the validation data for ERCOT system-wide. It is used to generate the plots and tables in section 3.4.

$\begin{array}{rrrr}\text { Actual } & \text { Persistence } & \text { Markov } & \text { ARMA } \\ 6953.88 & 6865.02 & 7693.56 & 6953.90 \\ 6964.82 & 6953.88 & 6533.42 & 6630.00 \\ 7560.1 & 6964.82 & 7458.04 & 7086.10 \\ 7505.61 & 7560.1 & 7458.04 & 7977.70 \\ 6859.51 & 7505.61 & 7693.56 & 7155.30 \\ 6468.97 & 6859.51 & 5800.70 & 6383.00 \\ 5894.84 & 6468.97 & 6594.48 & 6303.70 \\ 5142.95 & 5894.84 & 5190.10 & 5393.50 \\ 5158.75 & 5142.95 & 5190.10 & 4666.60 \\ 5382.66 & 5158.75 & 6289.18 & 5385.00 \\ 4778.69 & 5382.66 & 5190.10 & 5416.90 \\ 4119.7 & 4778.69 & 4359.68 & 4151.00 \\ 3718.63 & 4119.7 & 4050.31 & 3808.80 \\ 3719.3 & 3718.63 & 4050.31 & 3479.10 \\ 3374.31 & 3719.3 & 4335.26 & 3815.60 \\ 3338.69 & 3374.31 & 4335.26 & 2967.70 \\ 3664.04 & 3338.69 & 4050.31 & 3496.10 \\ 4704.71 & 3664.04 & 4945.86 & 3846.90 \\ 5844.89 & 4704.71 & 5678.58 & 5531.30 \\ 5544.6 & 5844.89 & 5556.46 & 6401.60 \\ 3903.39 & 5544.6 & 3317.59 & 4907.50 \\ 3566.71 & 3903.39 & 2808.76 & 2683.10 \\ 4104.15 & 3566.71 & 4359.68 & 3885.80 \\ 4187.59 & 4104.15 & 4274.20 & 4392.40 \\ 4629.46 & 4187.59 & 4762.68 & 4068.20 \\ 4816.23 & 4629.46 & 5190.10 & 5063.20 \\ 5215.44 & 4816.23 & 5190.10 & 4707.80 \\ 5214.61 & 5215.44 & 5190.10 & 5597.90 \\ 4434.27 & 5214.61 & 4884.80 & 4955.40 \\ 3892.21 & 4434.27 & 3317.59 & 3805.80 \\ 3425.81 & 3892.21 & 3968.90 & 3693.10 \\ 2940.78 & 3425.81 & 3480.42 & 3067.00 \\ 3084.9 & 2940.78 & 2442.40 & 2656.40 \\ 3718.1 & 3084.9 & 4050.31 & 3345.60 \\ 4074.4 & 3718.1 & 4359.68 & 4131.30\end{array}$

$\begin{array}{lrrr}4180.54 & 4074.4 & 4274.20 & 4142.40 \\ 4038.94 & 4180.54 & 4359.68 & 4202.80 \\ 3762.79 & 4038.94 & 4050.31 & 3856.40 \\ 3454.73 & 3762.79 & 3968.90 & 3569.20 \\ 3840.56 & 3454.73 & 3317.59 & 3240.30 \\ 4904.17 & 3840.56 & 5251.16 & 4281.40 \\ 5146.76 & 4904.17 & 5190.10 & 5609.30 \\ 5249.03 & 5146.76 & 5190.10 & 4938.90 \\ 5245.77 & 5249.03 & 5190.10 & 5408.40 \\ 5607.97 & 5245.77 & 5556.46 & 5105.90 \\ 5158.31 & 5607.97 & 5190.10 & 5969.30 \\ 5275.15 & 5158.31 & 5637.87 & 4492.30 \\ 4921.21 & 5275.15 & 5251.16 & 5698.30\end{array}$




\section{ReFERENCES}

[1] J.B. Cardell, C.L. Anderson, "Estimating the System Cost of Wind Power Forecast Uncertainty," in Power \& Energy Society General Meeting, 2009. (C) IEEE. doi: 10.1109/PES.2009.5275882.

[2] G. Mokryani, P. Siano, A. Piccolo, "Combined Monte Carlo Simulation and OPF to Evaluate the Market Impact of Wind Energy," in 8th Mediterranean Conference on Power Generation, Transmission, Distribution and Energy Conversion MEDPOWER 2012, Cagliari, 2012 (C) IET. doi: $10.1049 / \mathrm{cp} .2012 .2022$

[3] X. Lin, et al, "Evaluating the Impact on Transmission Capacity due to Wind Power" in Universities Power Engineering Conference (UPEC), 2012 47th International, London, 2012 (C) IEEE. doi: 10.1109/UPEC.2012.6398578

[4] P. Yu, B. Venkatesh, "A Practical Real-Time OPF Method Using New Triangular Approximate Model of Wind Electric Generators," in Power \& Energy Society General Meeting, 2009. C IEEE. doi: 10.1109/PESMG.2013.6672957.

[5] S. Rajagopalan, and S. Santoso, "Wind Power Forecasting and Error Analysis using the Autoregressive Moving Average Modeling," in Power \& Energy Society General Meeting, 2009. (C) IEEE. doi: 10.1109/PES.2009.5276019.

[6] A. Carpinone, R. Langella, and A.Testa, "Very Short-term Probabilistic Wind Power Forecasting based on Markov Chain Models," in Probabilistic Methods Applied to Power Systems (PMAPS), 2010. C IEEE. doi: 10.1109/PMAPS.2010.5528983

[7] N. Lu, et al, "A Comparison of Forecast Error Generators for Modeling Wind and Load Uncertainty," in Power \& Energy Society General Meeting, 2013. (C) IEEE. doi: 10.1109/PESMG.2013.6672978.

[8] M. Zhao, et al, "Research on wind power forecasting method using phase space reconstruction and artificial neural network," in Sustainable Power Generation and Supply, 2009. SUPERGEN '09. International Conference on, Nanjing, 2009 (C) IEEE. doi: 10.1109/SUPERGEN.2009.5348196

[9] A. Ghanbarzadeh, "Wind speed prediction based on simple meteorological data using artificial neural network," in Industrial Informatics, 2009. INDIN 2009. 7th IEEE International Conference on, Cardiff, 2009 C IEEE. doi: 10.1109/INDIN.2009.5195882 
[10] M. Li, Y. Pan, "Wind Power Prediction Based on BPNN and LSA," in Power and Energy Engineering Conference (APPEEC), 2012 Asia-Pacific, Shanghai, 2012 (C) IEEE. doi: 10.1109/APPEEC.2012.6307572

[11] A. Dukpa, I. Duggal, B. Venkatesh, and L. Chang "Optimal participation and risk mitigation of wind generators in an electricity market," IET Renewable Power Generation, February 2009.

[12] P. Gomes, and R. Castro, "Wind Speed and Wind Power Forecasting using Statistical Models: AutoRegressive Moving Average (ARMA) and Artificial Neural Networks (ANN)", International Journal of Sustainable Energy Development (IJSED), Vol. 1, Issues 1/2, March/June 2012.

[13] A. Hirotugu, "A new look at the statistical model identification", IEEE Transactions on Automatic Control, Vol. 19, (6): 716-723, 1974.

[14] C.M. Hurvich, and C.L Tsai, "Regression and time series model selection in small samples," Biometrika, vol. 76: 297-307, 1989.

[15] MATLAB Signal Processing ToolboxTM [Online]. Available: wwww.mathworks.com/access/helpdesk/help/toolbox/signal/ 